\title{
Insights into the Physics of Flapping Flight: Extending Axioms on Force Generation, Flow Features and Body Deformation in Insect Flight.
}

\author{
A Thesis \\ Presented to \\ the faculty of the School of Engineering and Applied Science \\ University of Virginia
}

In Partial Fulfillment
of the requirements for the Degree
Master of Science in Mechanical and Aerospace Engineering

By

Ayodeji Toluwanimi Bode-Oke

May 2017 


\section{APPROVAL SHEET}

The thesis is submitted in partial fulfillment of the requirements for the degree of Master of Science

Ayodeji T. Bode-Oke, Author

This thesis has been read and approved by the examining Committee:

\begin{tabular}{c}
\hline Haibo Dong, Advisor \\
\hline Gavin Garner, Committee Chair \\
\hline Hilary Bart-Smith, Committee Member \\
\hline Harsha Chelliah, Committee Member
\end{tabular}

Accepted for the School of Engineering and Applied Science:

Craig Benson, Dean

School of Engineering and Applied Science

May 2017 


\section{Table of Contents}

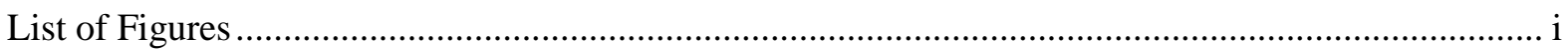

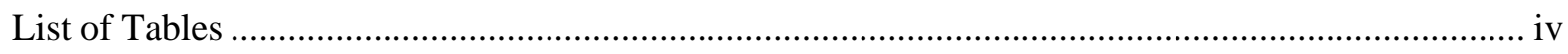

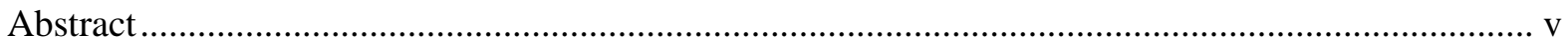

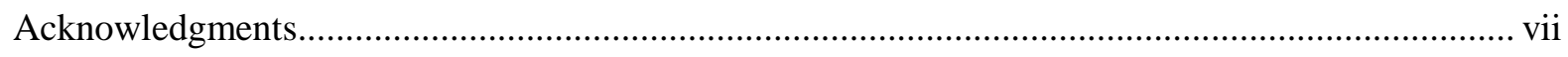

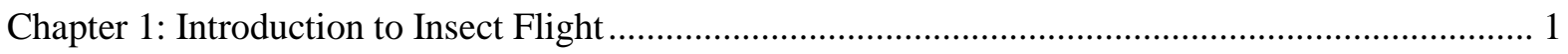

1.1 Flight in Nature and Advances in Insect Flight Studies....................................................... 3

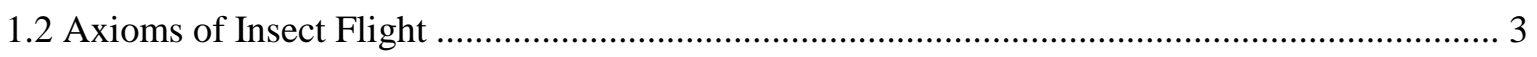

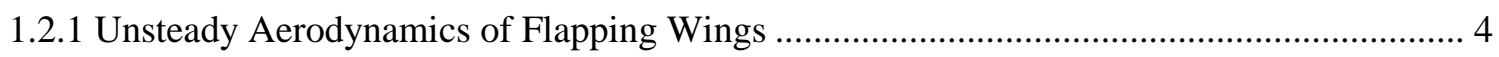

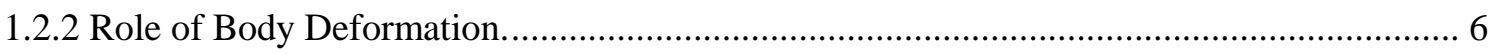

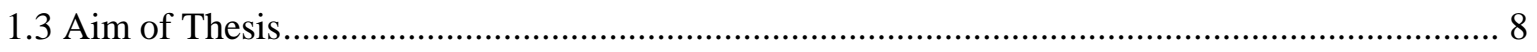

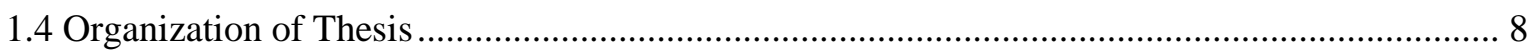

Chapter 2: Aerodynamic Force and Flow Features of a Damselfly (Hetaerina Americana) in Takeoff

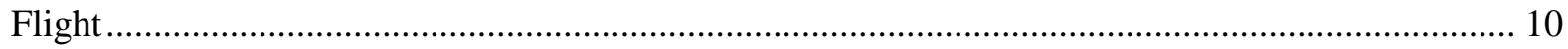

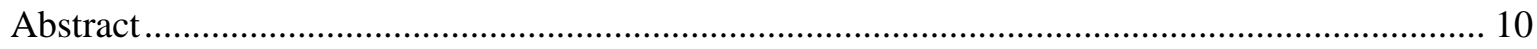

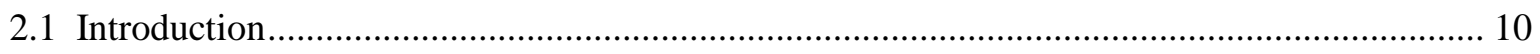

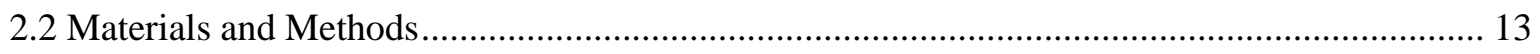

2.2.1 Damselflies, High speed photogrammetry and 3D surface reconstruction ........................ 13

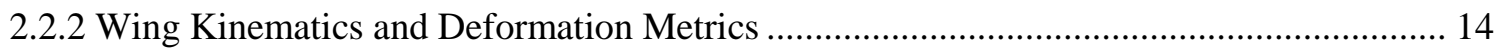

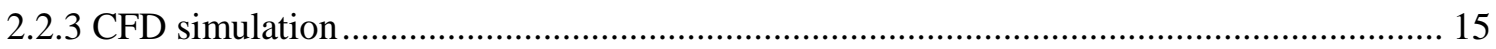

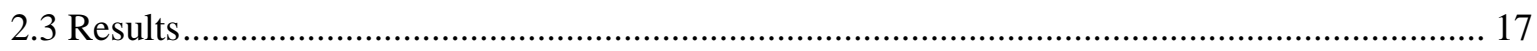

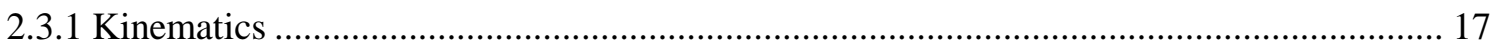

2.3.2 Identification of Vortical Structures during Takeoff Flight ............................................. 21

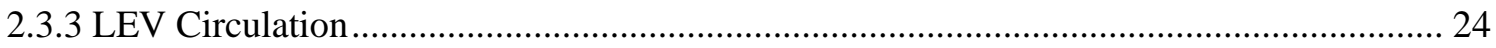

2.3.4 Pressure Distribution and Correlation to flow structures ................................................ 26

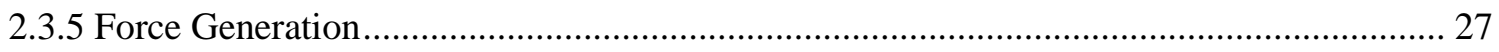

2.3.6 Effect of Wing-Wing Interaction (WWI) on Force generation........................................ 29

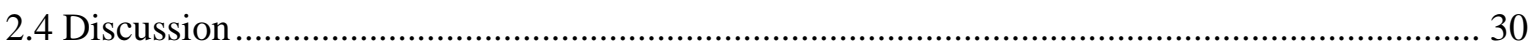

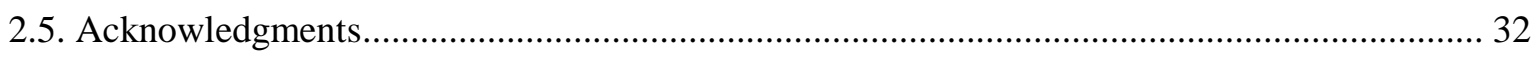

Chapter 3: Flying in Reverse: Kinematics and Aerodynamics of Dragonfly (Erythemis simplicicollis)

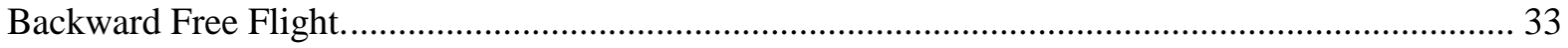

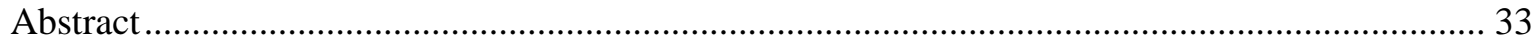

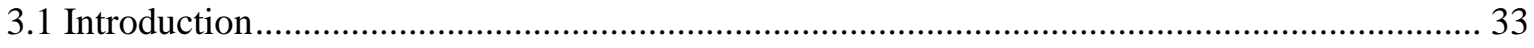

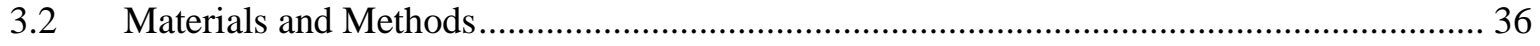


3.2.1 Dragonflies, High speed videography and 3D surface reconstruction .............................. 36

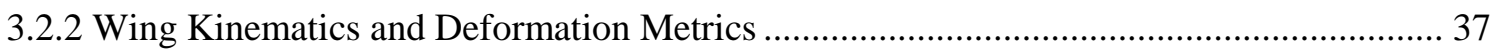

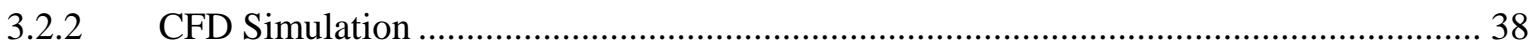

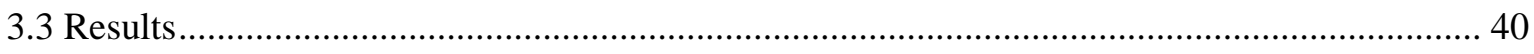

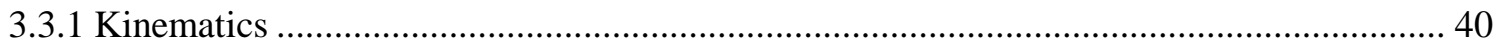

3.3.2 Identification of Vortical Structures during Backward Flight ........................................ 43

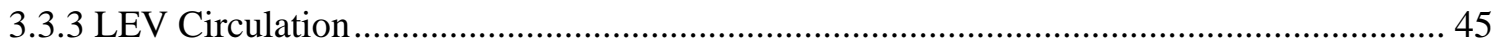

3.3.4 Force Generation: Aerodynamic Force and Power.......................................................... 47

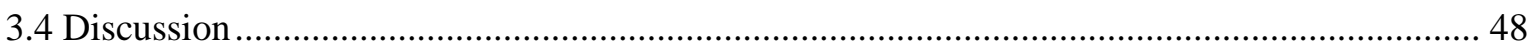

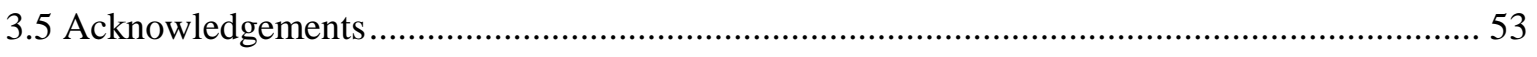

Chapter 4: Optimized Body Deformation in Dragonfly Maneuvers................................................ 53

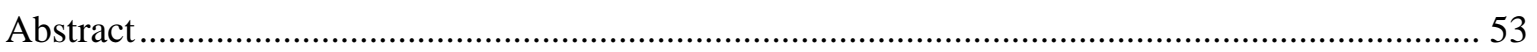

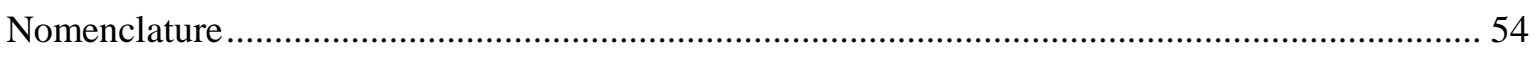

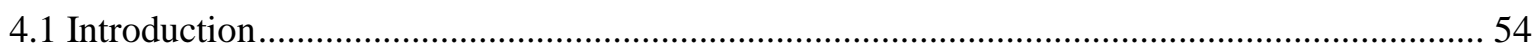

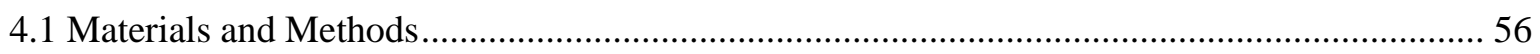

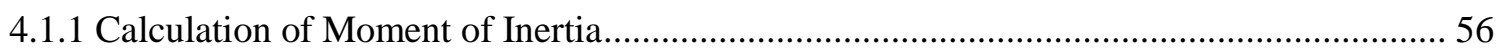

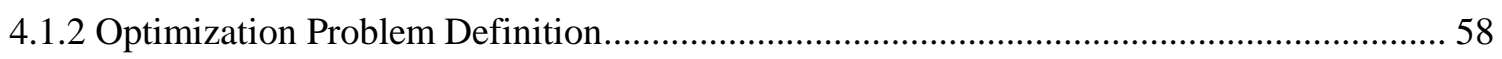

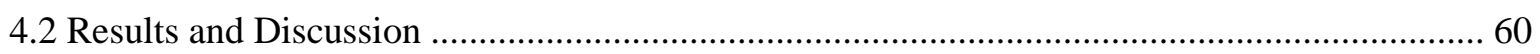

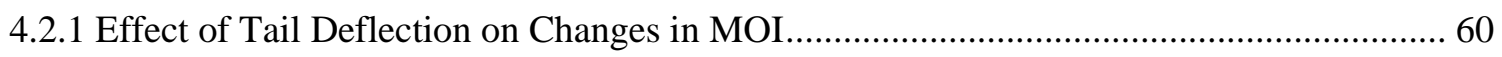

4.2.2 Optimized Tail Deflection and its Relationship to Body Yaw Velocity............................ 62

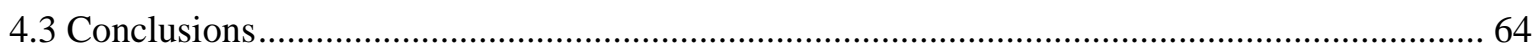

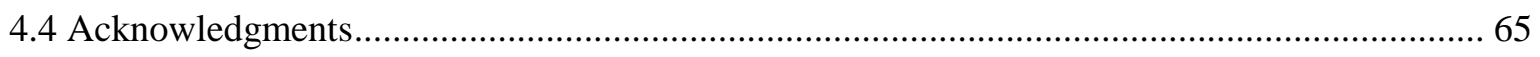

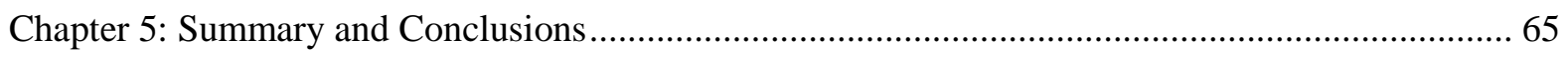

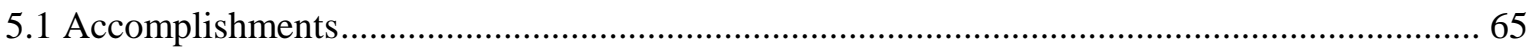

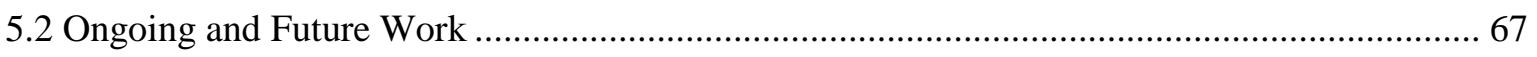

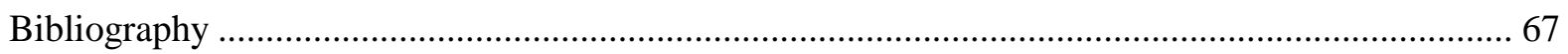




\section{List of Figures}

Figure 1: Ancient to Modern Flying Concepts. (A) Icarus with flying wings. (B) Leonardo da Vinci's Ornithopter model. (C) Wright Flyer I. (D) SR-71 Blackbird. (E) Quadcopter MAV. (F) Harvard's Robobee.

Figure 2: Leading Edge Vortex. (A) Flow over an airfoil (B) Leading Edge Vortex Suction Analogy (C) Leading edge vortex on a dynamically scaled robotic wing. Bubbles around the leading edge show the vortex for visualization purposes. Figure 2A,B were adapted from Sane [24]. Figure 2C was adapted from Lentink and Dickinson [17].....

Figure 3: Damselfly Species with Morphological Parameters. (A) A representative of the damselfly (Hetaerina Americana) in this study is shown. (B) An image of the reconstructed dragonfly is overlapped on the real damselfly image. The dots are the marker points placed on the wing for tracking the wing motion. Morphological parameters are also labelled. $\bar{c}$ is the mid-chord length, $\mathrm{L}$ is the damselfly body length, $\mathrm{R}$ is the wing length and $\mathrm{d}$ is the distance between the wing roots $(0.465 \bar{c}$ ).

Figure 4: Definition of the Kinematics Parameters. (A)Wing Kinematics. The geometric angle of attack $\left(\mathrm{AOA}_{\text {geom }}\right)$ is the angle between the normal to the chord (cyan arrow) and the normal to the stroke plane (purple arrow). The Euler angles of the wings in the stroke plane; flap, pitch and deviation are denoted by $\phi, \alpha$, and $\theta$ respectively. The green arrows represent a coordinate system placed at the wing root. (B) Body Kinematics. The coordinate system is fixed at the Centre of Mass. Roll, pitch and yaw are denoted by $\phi_{b}, \theta_{b}$ and $\psi_{b}$ respectively.

Figure 5: CFD Simulation Setup. (A)This figure shows the schematic of the computational mesh and boundary conditions employed in the current simulation for the Damselfly Flight. For ease of the display, the meshes are made less dense 4 times in each direction. (B) Grid Independent Study. The coefficient of lift is shown during the second flapping stroke. The gray shading indicates the downstroke of the forewings. Coarse $=9.8$ million; Medium=13.8 million; Fine $=15$ million. The medium grids are shown in (A)....

Figure 6: Kinematic events during an autonomous take-off in a damselfly (Hetaerina Americana; body weight $=0.735 \cdot \mathrm{mN}$ ). (A) A series of images depicting the sequence of events during the takeoff stroke. (B)Wing Kinematics. The kinematics are reported in the stroke plane. The definitions of the angles are in Figure 4. The angles of attack are reported at mid-span (C). Body Kinematics. The top figure shows the displament of the insect during flight. The bottom figure shows the body euler angles.

Figure 7: Local Twist and AOA Distribution from Mid-Span to Tip. (A)The local twist distribution and (B) AoA distribution at different spanwise locations (.50R,.70R,.90R) is reported here.

Figure 8: A time course of vortex development in the Takeoff Stroke. The vortical structures are visualized by the Q-criterion. The downstroke and upstroke are abbreviated as DS and US respectively.

Figure 9: Flow Features at mid-stroke visualized by the Q-criterion. (A) and (B) represents the flow features during the $1^{\text {st }}$ stroke at the fore and hindwing mid-strokes respectively. (C) and (D) represents the flow features during the $2^{\text {nd }}$ stroke at the fore and hindwing mid-strokes respectively. FW- Forewing, HW-Hindwing, TV-Tip Vortex, LEV- Leading Edge Vortex. Dashed circle in Figure 9D shows the LEV formed as the wing approaches reversal........................................................ 24 Figure 10: Quantitative measurement of Velocity and Pressure Difference on the Wing Surface. (A) Circulation at mid-Span of the left wings. (B) Pressure difference on the wing surface. (C) Isosurface of pressure coefficient $\left(C_{p}=-0.3\right)$.

Figure 11: Force generation. (A) and (B) show the time traces of vertical and horizontal forces, and resultant forces, generated by the wings. The blue line, black line and red line represent the forces 
generated by the forewing, hindwings, and the sum of the fore and hind wings, respectively. (C) is a rendering of the force vector whose components are shown in (A). The colors of the vectors correspond to the line in $(\mathrm{A})$.

Figure 12: Effect of Wing-Wing Interaction on Force generation. The dashed lines represent the cases when the wings are isolated from each other. The solid lines represent the case where all the wings were present in the CFD simulation.

Figure 13: Forward Flight versus Backward Flight. This figure shows the two possibilities of travelling from one point to another by the same dragonfly. (A) shows a takeoff maneuver in which the insect initiates flight, turns around and heads to its destination. (B) shows a backward flight initiation which does not necessitate a maneuver .

Figure 14: Kinematics Definitions. $\beta_{h}$ is the stroke plane with respect to the horizontal plane. $\beta_{b}$ is the stroke plane with respect to the longtiduinal axis of the body. $\phi, \theta, \alpha$ are the flap, pitch and deviation angles respectively. $\alpha_{\text {eff }}$ is the effective angle of attack. $\chi$ is the body attitude angle. It is the angle between the longitudinal axis of the body and the horizontal plane.

Figure 15: Computational Setup. (A) Schematic of the computational mesh and boundary conditions employed in the current simulation for the current study. For ease of the display, the meshes are made less dense 4 times in each direction. (B) Grid Independent Study. The coefficient of lift is shown during the second flapping stroke. The gray shading indicates the downstroke of the forewings. Coarse=10 million; Medium=14 million; Fine=16 million. The medium grids are shown in (A). The gray shading is based on the downstroke.

Figure 16: Body Motion During Backward Flight. (A) Orignal image reconstructed dragonfly is overlapped on the real damselfly image. The dots are the marker points placed on the wing for tracking the wing motion. (B) Snapshots (side and top views) of the dragonfly in backward flight during each wing beat. (C) 3D model of dragonfly in backward flight. (D) Body Kinematics during the flight. The gray shading here is based on the forewing timing.

Figure 17: Wing Kinematics and Deformation Metrics. The Euler angles as well as deformation (twist) are reported here. $\alpha_{\text {eff }}$ is reported at $0.70 \mathrm{R}$. The gray shading denotes the downstroke phase.43 Figure 18: Flow Features at Maximum Force Production. The vortical structures are visualized by the $\lambda_{2}$-criterion. FW- Forewing, HW-Hindwing, TV-Tip Vortex, LEV- Leading Edge Vortex, TEV-

Trailing Edge Vortex.

Figure 19: Leading Edge Vortex Circulation. (A) Time History of LEV during the $2^{\text {nd }}$ and $3^{\text {rd }}$ stroke. The gray shading denotes the downstroke phase for the forewings. (B)\&(C) Spanwise Distribution of LEV circulation at maximum force production during the $2^{\text {nd }}$ and $3^{\text {rd }}$ Stroke respectively.

Figure 20: Force Generation and Power Consumption. The aerodynamic forces and aerodynamic power consumption are reported here. The gray shading denotes the downstroke phase...... 48

Figure 21: Illustration of Forward Flight Versus Backward Flight. The length of the arrows denote the downstroke (green arrow) and upstroke (red arrow) forces based on their relative importance during (A) forward flight and (B) backward flight. The dashed lines indicate the inclined stroke plane. The black dot is placed at the bottom of the stroke plane in forward flight. By simply rotating the body to an upright posture, the black dot changes position to show the effect of body posture on rotation the stroke plane.

Figure 22: High Speed Photograph Indicating Tail Bending in Dragonflies. A selected sequence of images of a dragonfly in free flight is shown. (A) and (B) are images taken by the forward facing and downward facing cameras, respectively. The orientations of the thorax and the tail are indicated by white lines for clarity.

Figure 23: Dragonfly model for MOI calculations. Figure A depicts a sliced dragonfly model. The model is sliced into 21 pieces. Slices 1-3, 4-7, 8-21 make up the head, thorax, and abdomen, respectively. Figure $\mathrm{B}$ shows the approximation of an $\mathrm{i}^{\text {th }}$ slice as a cylinder having a volume 
equivalent to the slice. $r_{i}$ shows the corresponding radius of the approximated cylinder and $l_{i}$ shows the length of the cylinder. The length of the cylinder is equal to the length of each slice.

Figure 24: Optimization Problem Definition. Figure 24A shows a third order B-spline curve with 4 control points. The control points are depicted as red circles. The B-spline curve help generate the time history of the tail euler angles. Figure 24B represents the optimization flow chart. The inputs into the optimization algorithm are body density and length as well as body angular velocities, pqr. The volume of each slice of the dragonfly is obtained by dimensionalizing the non-dimensional volume by body length. Having the mass distribution, MOI and time rate of change of MOI can be calculated. The time rate of change of body angular velocities can be calculated also. The torque is calculated given angular velocities, rate of change of MOI, and rate of change of MOI. The cost function then calculates the energy expenditure. If the energy expenditure is minimal, the optimization algorithm output the tail angles and torques. Otherwise, the optimization is iterated by relocating the control points on the B-spline curves and the generating new tail euler angles. The process iterates till minimal energy expenditure is attained.

Figure 25: Effect of variation of tail Euler angles on MOI. The figures (A, B, C and D) show the amount of change that takes place in the moment of inertia when the tail is pitched only or yawed only. The tail angles are varied between -45 to 45 degrees. 0 degrees represents the erect position of the dragonfly. The top two figures, A and B, illustrate that when the moment of inertia is varied in pitch and yaw axis, the greatest variation occurs in the rolling moment of inertia. The rolling moment of inertia was about 20 times its original value while the pitching and yawing MOI did not change much. The bottom figures, $\mathrm{C}$ and $\mathrm{D}$, also illustrate the change in body symmetry when the tail is bent pitch wise or yaw wise.

Figure 26: Optimized tail bending kinematics and the resultant flight torque. This figure (A, B and $\mathrm{C}$ ) comprises of body angular velocity data, the optimized tail angles as well as the torques obtained from the cost function. Figure A, represent optimized results based on real insect flight data while $\mathrm{B}$ and $\mathrm{C}$ represent data obtained when the body yaw velocity was decreased to $75 \%$ and then $50 \%$, respectively. In the torque plots shown on the third row, the thick solid lines represent the rigid body torques, the thin dashed lines represent the aerodynamic torque and the thin solid lines represent the total flight torque. 


\section{List of Tables}

Table 1: Morphological data for the damselfly species in this experiment. The mass and length uncertainties are about $\pm 1 \mathrm{mg}$ and $\pm 1 \mathrm{~mm}$, respectively.

Table 2: Morphological Parameters for the Dragonfly in this Study. The mass and length measurement uncertainties are $\pm 1 \mathrm{mg}$ and $\pm 1 \mathrm{~mm}$ respectively.

Table 3: Quantification of Leading Edge Vortex Circulation. Table documents the LEV circulation US to DS ratio. $\bar{\Gamma}^{*}$ represents the time halfstroke averaged values. $\Gamma_{\max }^{*}$ represents the maximum circulation per halfstroke. All values are measured at $0.50 \mathrm{R}$

Table 4: Kinematic Parameters of Several Insects in Flight.

Table 5: Geometric Data of each slice of the dragonfly body. The non-dimensional length and volume of each slice of the dragonfly model are recorded. The non-dimensional MOI included in the table are non-dimensionalized by the body mass and length squared 


\begin{abstract}
In nature, only three organisms have been able to successfully achieve sustained flight; birds, bats and insects. Among these, insects are by far the most proficient and evolved flight over 300 million years ago. With advances in visualization techniques, high speed camera systems, experimental methods as well as computational fluid dynamics (CFD) simulations, Engineers have unraveled some of the mechanisms of flapping flight which govern aerodynamic force generation due to wing motions and body deformation.
\end{abstract}

The current consensus is that during flight, the downstroke flapping phase generates most of the aerodynamic forces with unsteady mechanisms, primarily, a leading edge vortex (LEV) aiding the forewings but this LEV is absent on the hindwings in the case of four-wing fliers. The upstroke is also mostly inactive and subsequently generates minimal aerodynamic forces during flight compared to the downstroke. However, these conclusions are based on well-studied flight modes such as forward flight, maneuvers and hovering and a considerable amount of the works were based on tethered flight. The coordination of wing and body motions and the role of unsteady aerodynamic mechanisms in other free flight modes such as flight initiation and reverse flight is not well known.

Auxiliary flight mechanisms such as body deformation may also influence flight mechanics. Most previous works on auxiliary flight mechanics have been based on observations in tethered flight where abdominal deflection may be exaggerated. Body deformation has been proposed as a mechanism for fine-tuning moments, ensuring pitch stability, modifying effective stability derivatives, and as an aerodynamic rudder during flight. However, it is not known whether there are energy savings benefits due to body deformation in free flight.

In this thesis, insight is provided on aerodynamic force generation, flow physics and body deformation during free flight from unique perspectives and methods. The roles of the wings in aerodynamic force generation in previously unstudied flight modes of four-winged fliers are investigated. Emphasis is placed on high-lift mechanisms employed during flight, the roles of the downstroke and more importantly the upstroke in generating flight forces, and the coordination of wing 
and body motion in flight. To this end, CFD simulations were employed to study novel flight modes which include non-jumping takeoffs in damselflies and backward flight in dragonflies. Additionally, an auxiliary flight mechanism such as body deformation was studied. The main question sought to be answered is whether there could be energy savings benefits from body deformation during free flight maneuvers. An optimization algorithm was employed to study body deformation in free flight maneuvers of Dragonflies.

The findings from this work advance knowledge on the physics of flapping flight by clarifying which mechanisms could benefit flight in different modes with relevant applications in the design of next generation robust MAVs. 


\section{Acknowledgments}

Firstly, I will like to thank all the great researchers and mentors before me who paved the way. I would also like to express my gratitude to my adviser Dr. Haibo Dong, Associate Professor of Mechanical and Aerospace Engineering at the University of Virginia (UVa). I will like to recognize all my colleagues at Flow Simulation Research Group who have played an important role in my development; Dr. Yan Ren, Dr. Geng Liu, Dr. Chengyu Li and Dr. Samane Zeyghami. Without them, this work would not exist. I especially thank Samane for her mentorship and friendship over these years. Samane and Dr. Dong were coauthors on all the works presented in this thesis which were a culmination of journal and conference paper manuscripts. They contributed to at least $30 \%$ of the intellectual information presented. I thank my thesis committee members; Dr. Gavin Garner, Dr. Hilary Bart-Smith and Dr. Harsha Chelliah as well as other faculty and staff in the Department of Mechanical and Aerospace Engineering, for their support. Dr. Kathryn Thornton, my undergraduate advisor, also gave me multiple opportunities to disseminate the work in this thesis as an avenue to get more students interested in the Aerospace program at UVa. I thank her a lot.

I appreciate my parents Olubode and Olusola as well as my siblings Ayomide and Ayotola sincerely. My family has always believed in me and have done everything possible so that I do not have to beg for bread in order to achieve my lifelong dreams. I love you very much. In addition, I also thank my friends for support outside the lab; Dr. Kimberley Bassett, Dr. John Gates, OneWay Christian Fellowship, The African Dance Ensemble at UVa, Freda Assuah, Chika Adiele, Sadiq Olanrewaju, Nicholas Doolittle, Fritz Bondoa, Taturah Byron, Audrey Ogendi and Tiffani Kennedy.

Most importantly, I thank God for seeing me through from birth till this phase of my life. He is the immortal invisible God to whom be praise and glory forevermore. 
In memory of my grandparents; Thomas Oladipo (1921-2008) and Esther Abeje (1935-2012) Oke. Esun re o. 


\section{Chapter 1: Introduction to Insect Flight}

"From Leonardo da Vinci to the Wright brothers, flight has inspired engineers more than any other form of animal behavior. Like any aircraft, an animal capable of active flight must possess three critical features: a light but powerful engine; wings capable of generating sufficient aerodynamic forces; and a control system to keep it from tumbling to the ground". - M. H. Dickinson [1]

Flying on a macro-scale used to be one of the greatest mysteries in human history. Since the beginning of time, man has been fascinated with the flapping flight of birds and this preoccupation has been documented in many ancient sources. In many ways, man has always looked to nature for inspiration in order to solve engineering problems. In Greek mythology, Daedalus and Icarus attempted to achieve flight with feathers fastened to their arms by wax. They were quite successful in escaping from the island of Crete but Icarus flew too close to the sun, the wax melted and he fell to his death [2]. The Babylonians, in their awe of flight, also opined that "To operate a flying machine is a great privilege. Knowledge of flying is most ancient, a gift from the gods of old for saving lives" [3]. Other common traditions about flight can be found in Indian mythology and Egyptian hieroglyphics respectively. And even in biblical traditions, angelic beings called the Cherubim, had six wings with two functional wings for flight. “...Above him were seraphim, each with six wings: With two wings they covered their faces, with two they covered their feet, and with two they were flying" (Isaiah 6:2).

Since the development of engineering concepts and mathematical principles, many have reattempted to achieve flapping flight. Leonardo da Vinci, designed ornithopters* in the 1400's but there is no evidence that they flew [2]. Even if they flew, they were not viable means for flight because unlike birds, humans do not have arm muscles evolved for flapping continuously. Hence one will fall to his death once one gets tired. Since flapping flight seemed unattainable to man, man had to resort to achieving flight by other means. The Montgolfier brothers flew the first lighter-than-air balloon (LTA) in 1783 and George Cayley in 1799, invented the fixed wing concept of flight. In Cayley's treatise, the fixed wing generated the lift, a separate mechanism provided thrust and vertical and horizontal tails

\footnotetext{
* Ornithopter is an aircraft that flies by flapping its wings.
} 
ensured stability. As a result of the groundbreaking work on gliders after Cayley's treatise, assisted takeoffs $^{\dagger}$ were possible and finally in 1903, the Wright brothers finally achieved the first heavier-thanair (HTA) flight in History [2]. Since then, man has expanded the boundaries of flight from subsonic to supersonic, fixed-wing to rotary, and recently flapping flight in the form of drones and Micro Aerial Vehicles (MAVs).
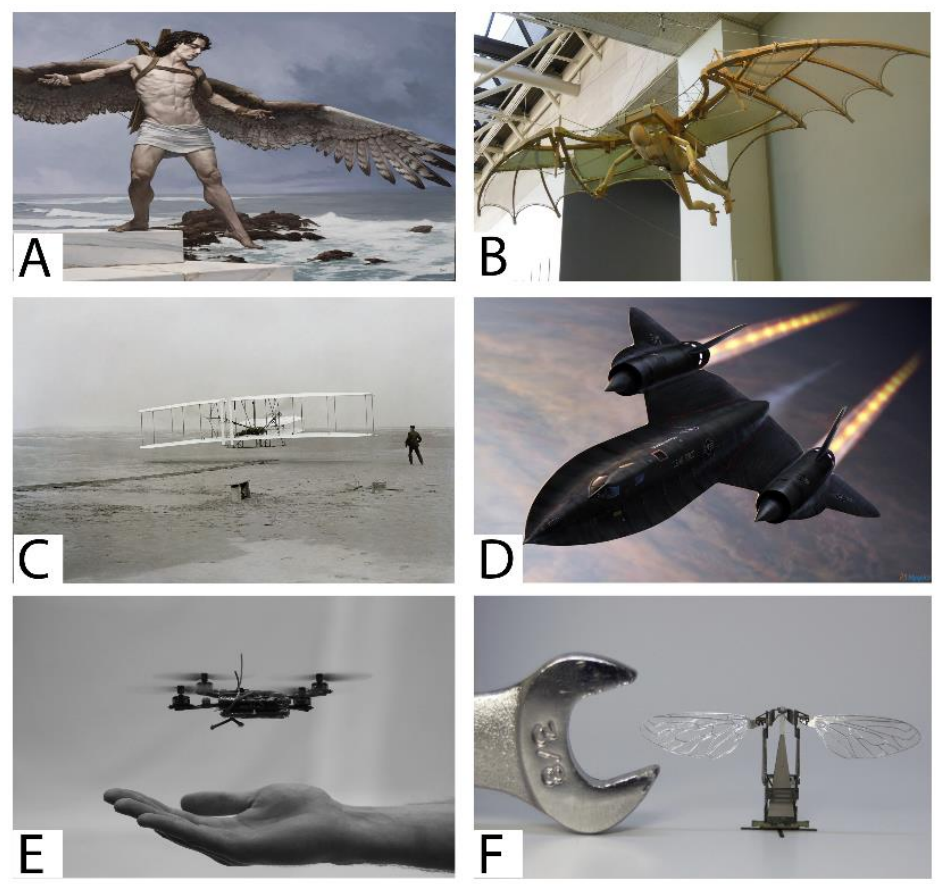

Figure 1: Ancient to Modern Flying Concepts. (A) Icarus with flying wings ${ }^{\ddagger}$. (B) Leonardo da Vinci’s Ornithopter model $^{\S}$. (C) Wright Flyer I**. (D) SR-71 Blackbird ${ }^{\dagger \dagger}$. (E) Quadcopter MAV ${ }^{\sharp 1}$. (F) Harvard's Robobee $\$$.

\footnotetext{
${ }^{\dagger}$ Assisted takeoffs are flights initiated from catapults or from hill.

${ }^{\star}$ https://dreamtravelblog.files.wordpress.com/2012/03/triumphstudy1.jpg

${ }^{\S} \mathrm{http}: / /$ rlkitterman.deviantart.com/art/Leonardo-da-Vinci-Ornithopter-479609315

${ }^{* * *}$ https://upload.wikimedia.org/wikipedia/commons/8/86/First_flight2.jpg

${ }^{\dagger \dagger}$ http://www.migflug.com/jetflights/remarkable-airplanes-of-the-world-part-1-the-fastest.html

$\mathrm{t}$ http://sensors.ini.uzh.ch/tl_files/sensors/pics/student\%20projects/Quadcopter.jpg

$\$$ http://therichest.imgix.net/2014/03/robobee-

feat.jpg?auto $=$ format $\& \mathrm{q}=100 \&$ lossless $=1 \& \mathrm{w}=1500 \& \mathrm{~h}=785 \&$ fit $=$ crop
} 


\subsection{Flight in Nature and Advances in Insect Flight Studies.}

According to evolutionary biologists, only three organisms have been able to successfully achieve sustained flight. They include birds, bats and insects. Among these, insects are by far the most proficient and were the first to evolve flight [4]. They have the ability to glide, fly very slowly and very fast, hover, evade looming predators in the blink of an eye and are capable of many other aerodynamics feats such as backward flight. Today, scientists and engineers are tasked with uncovering their secrets of flight and designing vehicles that can replicate their behavior. Research interest in the area of insect flight has peaked since the 1940s. However, most of the current understandings of insect flapping flight were made beginning in 1970s with the advent of better technologies such as Flapper apparatuses [5], Dynamically Scaled Robots $^{* * *}$ [4], High Speed Camera systems for observing insects in great detail and in slow motion, visualization techniques such as Particle Image Velocimetry (PIV) [6], low fidelity aerodynamic models such as Local Circulation Method (LCM) and Quasi-Steady Models [7, 8], and High Fidelity Computational Fluid Dynamics (CFD) tools $[9,10]$. A summary of major experimental and numerical studies in insect flight from 1970-2005 has been documented by Viieru et al. [11]. From those studies and many more within the last decade, several axioms have been developed to explain how insects fly.

\subsection{Axioms of Insect Flight}

Many axioms in insect flight have been able to explain how insects function from the perspective of force production (aerodynamics), flow physics (vortex dynamics), wing motion (kinematics), neurobiology, dynamics and control. However, here, emphasis is placed on aerodynamics, flow physics and body dynamics (body deformation). During flight, many insects such as dragonflies flap their wings while simultaneously deforming their bodies. Hence, two major

\footnotetext{
${ }^{* * *}$ Kinematics of insect is replayed on a larger sized robot tuned to have the same Reynolds Number as the insect.
} 
questions will be explored here. 1) How do insects generate forces for flight? ${ }^{\dagger \dagger \dagger}$ 2) If insects also deform their bodies while flapping, what is the role of body deformation (airframe morphing) during flight?

\subsubsection{Unsteady Aerodynamics of Flapping Wings}

Insects are nature's micro aerial vehicles (MAV). Hence, in order to design MAVs, it is important to understand flapping flight thoroughly. Insect fly by flapping their wings back and forth with frequencies ranging between $20-800 \mathrm{~Hz}$ [12]. Most of insect flight occurs in the laminar flow regime (Reynolds numbers, $10^{2}-10^{4}$ ) [13]. For large insects, the onset of turbulence occurs at Reynolds numbers $(\mathrm{Re})$ of $10^{4}[14]$. While beating their wings, insects are capable of varying the orientation of the wing surface along the stroke. The result of the reorientation of the wing surface is in the variation of the geometric angle of attack ${ }^{\dagger \ddagger}$ as well as the effective angle of attack $^{\S \S}$ during a flapping stroke. Insects generally flap at very high angles of attack compared to their fixed wing counterparts [14]. Under these conditions, an airfoil should stall and lose lift but somehow insects remain aloft. In addition, steady aerodynamics is insufficient to explain the flight of insect because the lift coefficients predicted are too small to sustain flight [8]. Rather, insect are able to utilize unsteady/high lift mechanism to sustain flight. These high lift mechanisms include Dynamic Stall/Absence of Stall, Rotation circulation, Wing-Wing/Wing Wake interactions, Clap and Fling [15].

In well-studied flight modes such as forward, maneuvering and hovering flight, the downward flap of the wing (downstroke) generates the lift forces for weight support. The wing excurses at high angles of attack during the downstroke. Flow separates at the sharp leading edge but reattaches before the trailing edge forming a leading edge vortex (LEV) (Figure 2). The LEV is a region of low pressure which enhances the bound circulation of the wing [16]. The LEV is stable and remains attached to the wing during excursion and sheds during reversal [17]. Depending on the Reynolds number, there may be a spanwise flow or the absence thereof in the LEV. Because of observations of a LEV on delta wings,

\footnotetext{
tit Majority of studies focus on wings as the primary agent for flight control and force modulation.

$\$$ Pitch of the wing along the path of the wings or stroke plane

$\S \S$ Angle between oncoming flow and the wing surface
} 
it was long thought that the spiraling flow in the LEV core is responsible for its stability. Birch and Dickinson [18] showed that a wing flapping at $\mathrm{Re}=120$ and $\mathrm{Re}=1400$ can generate a stable LEV. However, at low Re, spanwise flow is absent. Lentink and Dickinson [17], then showed that the stability of the LEV is due to the rotational acceleration of the wing, mainly the quasi-steady centripetal and Coriolis accelerations. They also showed that these mechanisms of LEV stability is independent of Re.

The leading edge vortex formed during translation is due to Dynamic stall/Delayed stall/Absence of stall and it is the most important high lift mechanism in insect flight. Ellington et al [19], demonstrated this finding using a smoke visualization on a flapping apparatus which replayed the kinematics of a hovering hawkmoth. Liu et al [9], further verified their observation using CFD simulations. Willmott et al [20] also visualized the LEV on tethered hawkmoths. Recently, Johansson et al [6], observed an LEV in free flight of hawkmoth and observed a dual-core structure using PIV. Up until this point, all observations were made on tethered flight or flapping apparatus or model problems. For more information of leading edge vortex topology, see [21-23]. Due to rapid changes in angle of attack at reversal, another leading edge vortex could be formed due to rotational effects [19]. Other high lift mechanisms such interactions between the wings due to their proximity as well as with the previous wake may be beneficial to flight [24]. The LEV has been observed mostly in the downstroke flapping phase.

The role of the upstroke (US) in flapping flight is somewhat controversial. The major conundrum is whether the upstroke is active or not. An active upstroke means that the upstroke can generate substantial forces for weight support or thrust production. Normally, the upstroke only generates forces that are at most $10-20 \%$ of the body weight [25]. The only exceptions are in the hovering flight of Drosophila and Mosquitos where the US generates majority of the force for weight support $[12,26]$. In four-wing fliers, such as dragonflies reverse is the case. Unlike the downstroke (DS), most studies have reported the absence of an LEV during the upstroke [27]. The reason is that during the upstroke, the wing slices through the air like a knife, resulting in very low effective angles of attack. Wang and Russell [28] measured these angles to be as low as $8^{\circ}$ during hovering flight of 
dragonflies. Even under steady conditions, a conventional airfoil will not stall at this angle. Hence the absence of an LEV during the upstroke. Wang and Sun [27], using computational simulations also verified the absence of the LEV in the upstroke in hovering as well as forward flight. Using smoke visualization in a wind tunnel, Thomas and Taylor [21] showed that dragonflies only utilized an LEV on the forewings during the downstroke phase. They reported attached flows on dragonfly hindwings as well as during the upstroke phase of flight in forward flight. Using a similar smoke visualization technique, Willmott et al. [20], also showed the absence of an LEV during the upstroke of hawkmoths in forward flight.
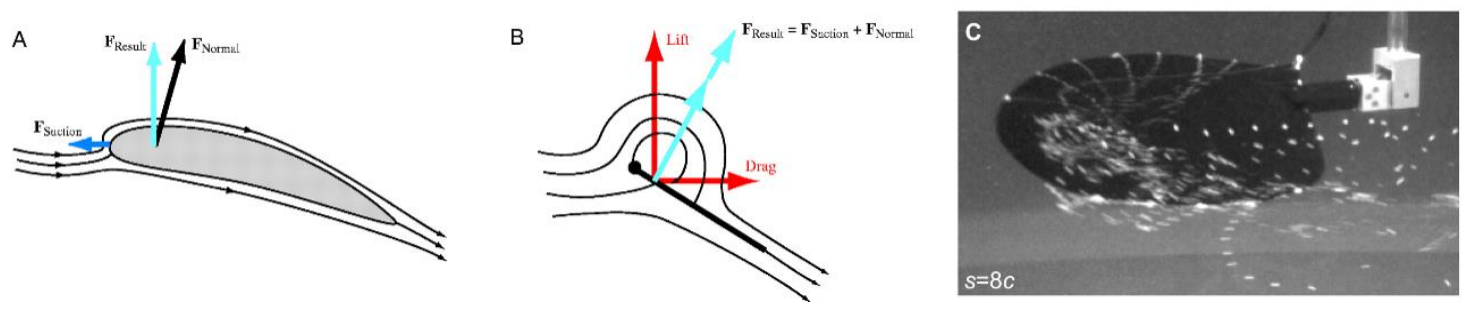

Figure 2: Leading Edge Vortex. (A) Flow over an airfoil (B) Leading Edge Vortex Suction Analogy (C) Leading edge vortex on a dynamically scaled robotic wing. Bubbles around the leading edge show the vortex for visualization purposes. Figure 2A,B were adapted from Sane [24]. Figure 2C was adapted from Lentink and Dickinson [17].

While all these aforementioned studies have helped foster the understanding of insect flight, majority of the work has been based on specific flight modes primarily forward flights and hovering. Many experimental studies have also been based on tethered flight. It is not known whether the same aerodynamic mechanisms, flow features and wing motions apply to other flight modes such as flight initiation (takeoff) and backward flight.

\subsubsection{Role of Body Deformation.}

As insect flap their wings, they also morph their bodies. Although less attention has been paid to body deformation (airframe morphing), there have been pioneering studies over the years to uncover 
its relevance in flight; especially in flight control. Body deformation has been observed in a wide range of insects from Desert Locusts (Schistocerca gregaria) [29, 30], Fruit flies (Drosophila melanogaster) [31], Moths (Manduca sexta) [32], Honey Bees (Apis Mellifera) [33]. The primary take away here is that an insect's body may serve as an auxiliary mechanism to effect aerodynamic, dynamic and control changes during flight in addition to input from the wings. Airframe morphing usually is a compensatory behavior based on stimuli. Airframe morphing can be elicited in animals by visual stimuli as in the case of moths and honeybees or external stimuli such as wind direction in the case of locusts. Camhi [29] first observed that by angling the incoming direction of wind toward a tethered locust, the animal deflected it abdomen by almost the same angle as the wind direction i.e. a somewhat linear response was elicited for yaw correction. He further demonstrated in another work [30] that flying locusts use their abdomen as aerodynamic rudders. In essence, the drag produced on the body due to deflection can lead to increased moments (drag-based yaw torques). However, in a recent publication, Berthe and Lehmann [34] showed that abdominal deflection is for the purpose of fine-tuning moments rather than generating large moment during maneuvers (saccades) of fruit flies.

Another implication of airframe morphing is that it shifts the center of mass relative to the center of pressure, to produce lateral moments. Although, this should produce a roll-torque, in hangingpendulums such as fruit flies (body angle $\sim 30-50^{\circ}$ ), it induces a yaw moment [35]. Luu et al. [33], by visually stimulating (virtual reality) forward motion in honey bees observed that instead of leaving the abdomen hanging at an angle to the head and thorax, the insects actively aligned the abdomen to the thorax to achieve greater streamlining. During free flight, the streamlining of the abdomen could reduce drag. Dyhr et al. [32], approached the body deformation problem from a systems identification approach in order to quantify the stability or agility of the system which was a hovering hawkmoth. From their analysis, they observed that the experimentally measured body deformations was necessary for ensuring pitch stability during hovering flight. Results were also corroborated by Taylor [35]. Finally, Zeyghami et al. [36], demonstrated that without wing kinematics changes, the agility and 
stability of the system can be modified through body deformations which changes the MOI and effective stability derivatives.

While all these aforementioned studies have helped foster the understanding of body deformation, most studies were performed on tethered flight where abdominal responses may be exaggerated. In addition, there exists no study of body deformation in dragonflies although dragonflies display obvious body deformations during flight. For these highly skilled fliers, could body deformation be important and could they be using body deformation to achieve another effect during flight other than stability, such as energy minimization?

\subsection{Aim of Thesis}

The aim of this thesis is to reexamine the aforementioned current axioms in insect flight and perform seminal investigations on new flight modes using computational approaches, both CFD and an optimization algorithm, to ascertain which rules govern flight in different modes in four-winged fliers. The major question sought to be answered in this thesis are as follows. 1) How do kinematics and aerodynamic/flow mechanisms change when flight mode changes? 2) If an insect deflects its abdomen during flight for maneuvering, what is the role of body deformation? Could airframe morphing be beneficial? To answer these questions, the mechanisms of force generation (aerodynamics), vortex dynamics (flow features) and particularly the roles of the upstroke and downstroke were investigated by considering less studied flight modes such as non-jumping takeoffs in damselflies and backward flight in dragonflies. In addition, the role of body deformation during dragonfly maneuvers was investigated.

\subsection{Organization of Thesis}

Chapters 2 and 3, characterize kinematics, force generation and unsteady aerodynamic mechanisms in new flight modes. Chapter 2 investigates, for the first time, the aerodynamic force production and flow features during non-jumping takeoffs of a damselfly. Chapter 3 focuses, for the 
first time, on the kinematics, aerodynamics and flow features of a dragonfly in backward flight. As insects flap their wings, they also deform their bodies. To close the loop, Chapter 4 examine the role of body deformation in flight. The problem is studied using a novel optimization algorithm. In all the chapters of this thesis, Four-winged fliers (evolved 300 million years ago) are used as case-studies. Chapter 5 presents the accomplishments of the thesis. Current and future research efforts are also enumerated. 


\title{
Chapter 2: Aerodynamic Force and Flow Features of a Damselfly (Hetaerina Americana) in Takeoff Flight
}

\begin{abstract}
Here, for the first time, using a high fidelity CFD simulation, we identified the three dimensional flow features of a damselfly during a non-jumping takeoff. The damselfly initiated flight from a steeply pitched down orientation $\left(-50^{\circ}\right)$ and flew up and forward thereafter. Our results show that a damselfly is capable of generating about 3 times its body weight during the first halfstroke. In generating forces, the wings flap through a steeply inclined stroke plane with respect to the horizon, slicing through the air at high angles of attack. Consequently, a leading edge vortex (LEV) is formed on both the fore and hind wings. During takeoff, we discovered that an LEV of equal strength (circulation) is formed both in the down and up strokes in the initial wing beat (WB). Thus, indicating the presence of both an active upstroke and downstroke. Comparing the upstroke forces in the takeoff stroke versus subsequent strokes where no LEV was formed, we hypothesize that the LEV helped substantially boost force production on the wing surface. Our work shows that damselflies are capable, depending on the flight scenario or challenge, to switch between an active and an inactive upstroke to maximize force generation.
\end{abstract}

Keywords: takeoff flight, Damselfly, low Re aerodynamics, leading edge vortex (LEV), active upstroke, flight initiation.

\subsection{Introduction}

Most forms of flight are initiated by a takeoff. From airplanes to birds, bats and insects, all share this common behavior. Particularly, for living organisms, takeoff is essential for survival, escape from predators and lifting payload from one flower to another during pollination, etc. For scientists and engineers, emphasis is placed on understanding the behavioral mechanisms during flight initiation in order to apply the findings to applications such as micro-aerial vehicle (MAV) design [37].

In understanding flight initiation, a lot of research has focused on jumping takeoffs of locusts [38], crickets [39], fruit flies [40], leafhopper insects [41] and birds[42, 43]. However, a few studies 
have analyzed non-jumping takeoffs of insects. During non-jumping takeoffs, majority of the forces for flight are generated by the wings and the body motion is slow in comparison to those achieved during jumping takeoffs. Furthermore, most slow takeoffs are stable. Takeoff is considered stable if the rotational velocities of the body are not too high to induce tumbling in the insect [40, 44].

Truong et al. [45], analyzed a non-jumping takeoff of a rhinoceros beetle (flapping frequency $\sim 37-38 \mathrm{~Hz}$ ). The authors observed that takeoff did not occur during the first flapping stroke but at the end of the 3rd wing beat (WB). The impulse produced during the first wing beat was not sufficient to ensure liftoff. By modulating the pitch angle/geometric angle of attack ( $\left.\mathrm{AOA}_{\text {geom }}\right)$ of the wing and increasing flapping frequency, the insect achieved liftoff with the hindwings generating most of the necessary forces for flight. Additionally, Chen et al. [44], studied a smooth slow takeoff of drone flies. For this high flapping frequency insect $(\sim 170 \mathrm{~Hz})$, the legs left the ground after the $13^{\text {th }}$ wing beat. Prior to liftoff, the aerodynamic forces increased to a value greater than the body weight while the flapping amplitudes also increased gradually and the leg forces decreased monotonically. In both papers ([44] and [45]), the insects pitched up while ascending during flight initiation. However, these studies although essential, did not identify the important flow features as well as the connection between the wing kinematics and aerodynamics mechanisms that enabled flight initiation.

Here, we study the takeoff of a damselfly ( 27-28 Hz). Damselflies are known to initiate flight solely by flapping their wings [46]. However, none but one study has focused on an aspect of the takeoff mechanism of damselflies. Marden [46], measured the maximum payload a damselfly can carry during takeoff to determine the maximum lift production of the wings in still air. The maximum lift per unit mass ranged between 21.5-50 N/kg. Nevertheless, no information on the wing kinematics or further aerodynamic data was provided.

To understand the mechanics of takeoff, we enumerate some findings from previous literature on forward and hovering flight of true four-winged fliers; Odonata and Zygoptera, to which we can later compare our findings. During forward flight, counterstroking wherein the hindwings lead the forewings is the normal flight mode preferred by dragonflies [21, 47, 48]. In contrast, Zygoptera fly 
forward with the forewings leading the hindwings by quarter of a cycle [7]. In both insects, the stroke planes are inclined and the majority of the force is generated in the downstroke during which the wings flap with higher pitch angles [49]. The upstroke, however, is primarily for thrust production, generating minimal forces about $10-20 \%$ of the body weight $[8,19,25,47,50]$. In regard to body posture, during hovering flight, the dragonfly body is horizontal while in forward flight the body angle is dependent on the flight speed [51]. Body angle reduces with increasing speed in order to reduce parasitic drag [25, $52,53]$.

To reconcile the relationship between the flapping motion of wings and the corresponding force production, some unsteady aerodynamic mechanisms have been suggested such as absence of stall/delayed stall, clap and fling, rotational circulation and wing-wake interactions [24]. Among these, the leading edge vortex (LEV) formed during delayed stall is the most potent and has been observed in multiple insect species such as hawkmoths[6], bumblebees[54], and dragonflies [21]. The LEV is responsible for about two-thirds of the total lift force in insect flight [55] but has just recently been measured in free flying hawkmoths in forward flight [6]. All previous studies have been based on tethered flight. The LEV is a region of low pressure formed on the upper part of the wing surface toward the leading edge and it enhances lift by adding its circulation to the bound circulation of the wing [16]. An LEV is formed either during translation or rotation of the wing during reversal. When a wing excurses at high angle of attack, flow separates at the leading edge and reattaches to the wing surface before the trailing edge. The wing has dynamically stalled [15]. During this period, the LEV is stably attached to the wings surface and breaks down as the wing approaches the end of the stroke. In the course of rotation, an LEV can also be formed. Rapid increases in angles of attack during reversal could lead to the formation of an LEV. So far, the presence of LEVs has been extensively reported in the downstroke phase of flight. Conversely, a small LEV or attached flow has been reported during the upstroke phase of flight as well as on the hindwings of dragonflies [19, 21, 56].

Apart from the LEV, another important unsteady mechanism relevant to takeoff is "clap and fling”. Fling was observed as a mechanism for generating large forces in butterfly takeoff [57]. Fling 
eliminates slow development of circulation but creates large circulation values independent of wing translation or the Wagner effect $[24,58]$. When weights were attached to damselflies during takeoff, they used the mechanism [46]. However, in free flight with no attached payload, the mechanism was not used [7, 25]. Other insects also choose to shun this mechanism [25].

Our work adds to a handful of studies on damselflies (Zygoptera) flight mechanics [7, 46, 59]. Previously used quantifications of force and unsteady effects involve the local circulation method (LCM) for circulation measurement and quasi-steady models for approximating the force [7, 52, 59]. PIV has also been used to study tethered flight of insects, the free flight of birds and bats and recently free flight of Hawkmoth and dragonflies [6, 42, 55, 56, 60-62]. For the first time, however, using a high fidelity CFD simulation, we are able to identify the 3D flow features of a damselfly during takeoff. Furthermore, we discuss unique wing and body kinematics not previously mentioned in damselfly literature. And finally, we elucidate how the kinematics influences the aerodynamics to ensure a successful flight.

\subsection{Materials and Methods}

\subsubsection{Damselflies, High speed photogrammetry and 3D surface reconstruction}

Damselflies (Hetaerina Americana) were caught outside during the summer of 2012 in Dayton, Ohio for experiments. Prior to the experiments, the caught damselflies' wings were dotted for tracking purposes (Figure 3). The video footages were then recorded shortly after their capture in a quiescent environment of about room temperature $\left(70^{\circ} \mathrm{F}\right)$ in order to prevent inactivity during the time of experiments.

Table 1: Morphological data for the damselfly species in this experiment. The mass and length uncertainties are about $\pm 1 \mathrm{mg}$ and $\pm 1 \mathrm{~mm}$, respectively.

\begin{tabular}{ccccccccc}
\hline \hline Species & $\begin{array}{c}\text { Body } \\
\text { weight } \\
(\mathrm{mg})\end{array}$ & $\begin{array}{c}\text { Body } \\
\text { length } \\
(\mathrm{mm})\end{array}$ & $\begin{array}{c}\text { Forewing } \\
\text { length } \\
(\mathrm{mm})\end{array}$ & $\begin{array}{c}\text { Forewing } \\
\text { chord } \\
(\mathrm{mm})\end{array}$ & $\begin{array}{c}\text { Hindwing } \\
\text { length } \\
(\mathrm{mm})\end{array}$ & $\begin{array}{c}\text { Hindwing } \\
\text { chord } \\
(\mathrm{mm})\end{array}$ & $\begin{array}{c}\text { Wing } \\
\text { Area } \\
\left(\mathrm{mm}^{2}\right)\end{array}$ & $\begin{array}{c}\text { Flapping } \\
\text { frequency } \\
(\mathrm{Hz})\end{array}$ \\
\hline \hline $\begin{array}{c}\text { Hetaerina } \\
\text { Americana }\end{array}$ & 75 & 42 & 29 & 6 & 29 & 6 & 136 & 27
\end{tabular}


The damselflies initiated flight autonomously in the shooting area. Their flight triggered a photogrammetry setup consisting of three orthogonally arranged and synchronized cameras shooting at 1000 frames per second (fps). After recording the flight, the wings were removed and morphological parameters were measured. We selected one of the recorded footages for analysis. In this recording, the damselfly initiated flight from a pitch down body orientation and flew upward and forward thereafter. The dotted wings of the insect were tracked manually frame by frame in Autodesk Maya (Autodesk Inc.). Figure 3B shows a visual representation of the reconstructed insect placed on top of an image. The accuracy of this reconstruction technique is discussed extensively in [63] and has been applied in the study of damselflies and cicadas [10, 59, 64].
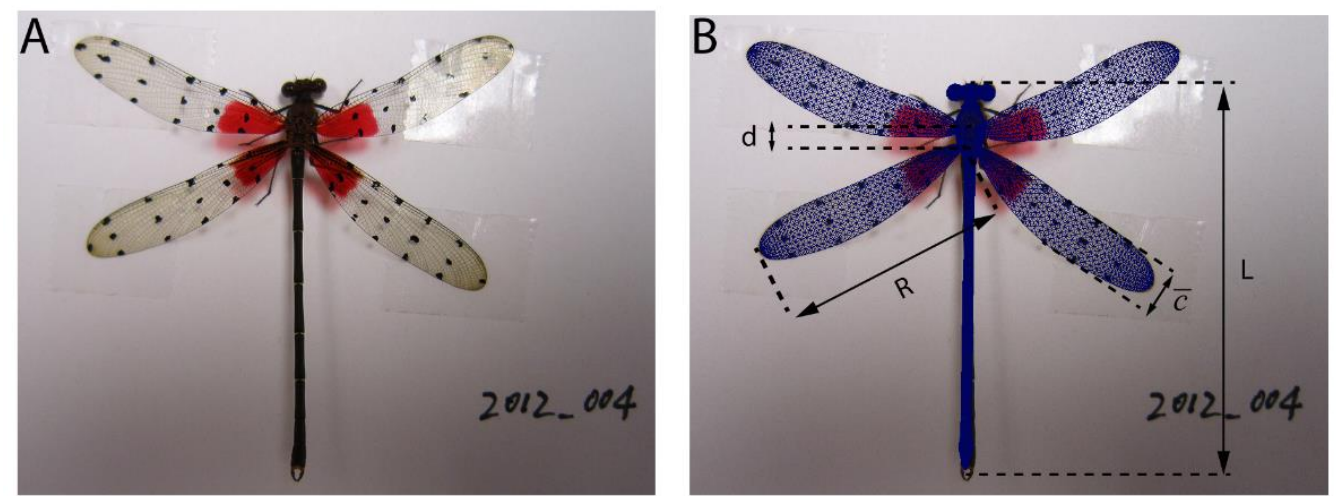

Figure 3: Damselfly Species with Morphological Parameters. (A) A representative of the damselfly (Hetaerina Americana) in this study is shown. (B) An image of the reconstructed dragonfly is overlapped on the real damselfly image. The dots are the marker points placed on the wing for tracking the wing motion. Morphological parameters are also labelled. $\bar{c}$ is the mid-chord length, $\mathrm{L}$ is the damselfly body length, $\mathrm{R}$ is the wing length and $\mathrm{d}$ is the distance between the wing roots $(0.465 \bar{c})$.

\subsubsection{Wing Kinematics and Deformation Metrics}

The wing kinematics and deformations were measured by defining a least-square reference plane (LSRP) generated based on the points on the reconstructed wing surface. The LSRP is the plane of least wing deformation. For details of how the LSRP was calculated see the work of Koehler et al. [63]. A mean stroke plane was also defined based on the average trajectory wing tip trajectories per stroke. The orientation of the wing in the stroke plane is described by three Euler angles; flap, deviation 
and pitch. Flapping motion is the revolution of the wing in a back and forth manner and is positive during the downstroke. The up and down rotations with respect to the stroke plane is expressed by the deviation angle as is positive when the wing is above the stroke plane. The wing pitch angle is characterized by the rotation of the wing about its pitching axis close to the leading edge. The pitch angle is measured clockwise from the intersection between the LSRP and the line defining the stroke plane hence varies between $0^{\circ}$ to $180^{\circ}$. The geometric angle of attack $\left(\mathrm{AoA}_{\text {geom }}\right)$, is defined as the angle between the normal to LSRP and the normal to the stroke plane. It is equivalent to the wing pitch. The effective angle of attack $\left(\mathrm{AoA}_{\text {eff }}\right)$, is the angle between the wing and the incident flow upon it. For wing deformation metrics, the local twist angle is reported. Visual representation of these definitions is rendered in Figure 4.
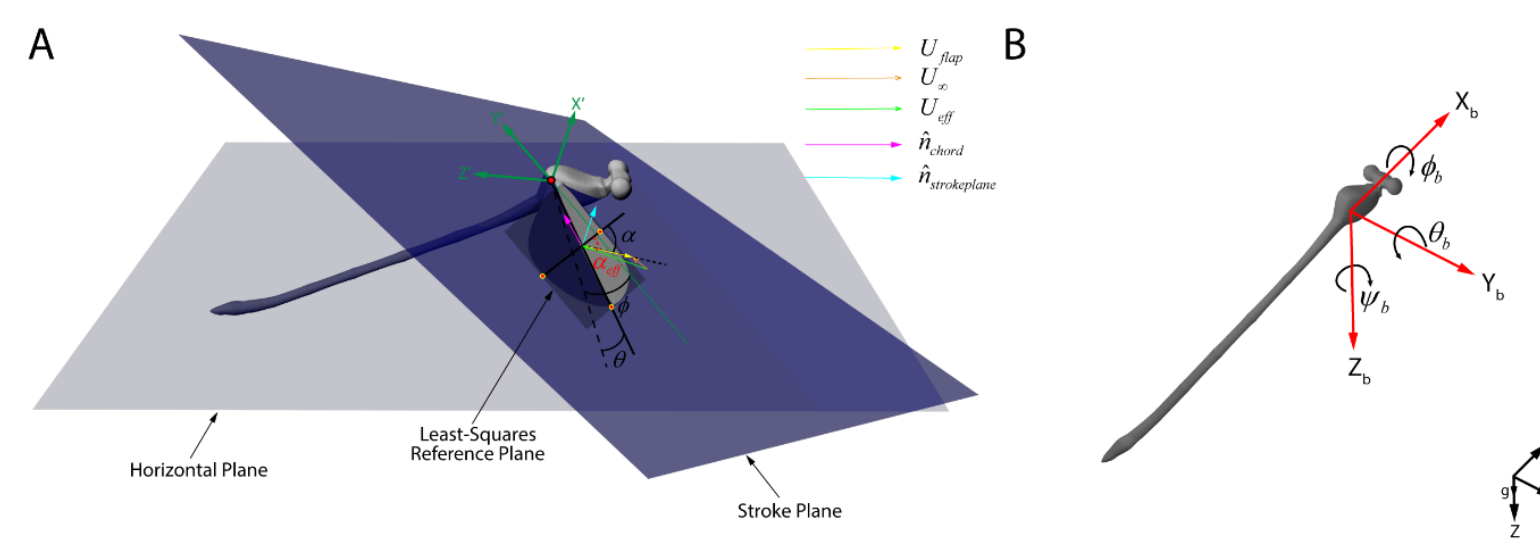

Figure 4: Definition of the Kinematics Parameters. (A)Wing Kinematics. The geometric angle of attack $\left(\mathrm{AOA}_{\text {geom }}\right)$ is the angle between the normal to the chord (cyan arrow) and the normal to the stroke plane (purple arrow). The Euler angles of the wings in the stroke plane; flap, pitch and deviation are denoted by $\phi, \alpha$, and $\theta$ respectively. The green arrows represent a coordinate system placed at the wing root. (B) Body Kinematics. The coordinate system is fixed at the Centre of Mass. Roll, pitch and yaw are denoted by $\phi_{b}, \theta_{b}$ and $\psi_{b}$ respectively.

\subsubsection{CFD simulation}

To elucidate the flow field around the damselfly in flight, we obtained the flow information from computational simulations. A high fidelity CFD solver based on the immersed boundary method was used in this study. We solved the incompressible Navier-Stokes Equation (Eqn.(1)) using a finite difference method with $2^{\text {nd }}$ order accuracy in space and a $2^{\text {nd }}$ order fractional step methods for 
progressing in time. More details of this method and its application in studies on dragonflies and cicadas can be found in the works of Liu et al., Koehler et al., and Wan et al. [10, 63, 65].

$$
\nabla \cdot \mathbf{u}=0 ; \quad \frac{\partial \mathbf{u}}{\partial t}+\mathbf{u} \cdot \nabla \mathbf{u}=-\frac{1}{\rho} \nabla p+v \nabla^{2} \mathbf{u}
$$

The vortex structures were characterized by the Q-criterion [66] shown below.

$$
Q=\frac{1}{2}\left[|\boldsymbol{\Omega}|^{2}-|\mathbf{S}|^{2}\right]>0
$$

Where $\mathbf{S}=\frac{1}{2}\left[\nabla \mathbf{u}+(\nabla \mathbf{u})^{\mathrm{T}}\right]$ and $\boldsymbol{\Omega}=\frac{1}{2}\left[\nabla \mathbf{u}-(\nabla \mathbf{u})^{\mathrm{T}}\right]$ are the strain rate and vorticity tensors respectively. The simulation was run on a non-uniform Cartesian grid. The computational domain size was about 50 $\bar{c} \times 50 \bar{c} \times 50 \bar{c}$ with about 13.8 million grid points in total. The high resolution uniform grids surround the insect in a volume of $14.5 \bar{c} \times 12 \bar{c} \times 13.5 \bar{c}$ with a spacing of about $0.06 \bar{c}$. This ensured sufficient resolution for capturing the 3D flow field. The stretching grids move in all three directions from the fine region to the outermost boundaries. The boundary conditions of the domain on all sides are zero gradient.

The Reynolds number for the flight is defined by $\operatorname{Re}=\frac{\bar{U}_{t i p} \bar{c}}{v}$ and is about 1220. It is measured based on the average wing tip speed $\left(\bar{U}_{t i p}=3.05 \mathrm{~m} / \mathrm{s}\right)$, mid-chord length $(\bar{c}=0.006 \mathrm{~m})$, kinematic viscosity of air at room temperature $\left(v=1.5 \times 10^{-5} \mathrm{~m}^{2} \mathrm{~s}^{-1}\right)$. Validation for the flow solver can be found in another recent work [65].

The current grid set-up was selected based on checks to ensure the grids are refined to preclude grid dependence of the simulation. Figure 5B shows the comparison of the lift coefficient history of the second flapping stroke in three grids (coarse, medium and fine). The difference of both the mean value and the peak value of lift between the medium-grid case (adopted in this paper) and the fine-grid case is about $2 \%$ thereby, establishing that the aerodynamic force calculations in the current study were gridindependent. 
A

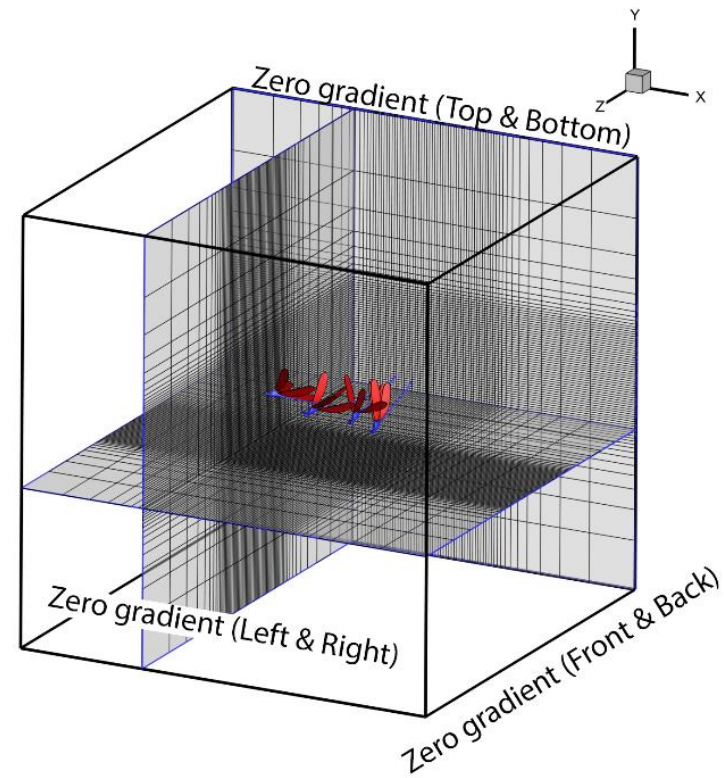

B

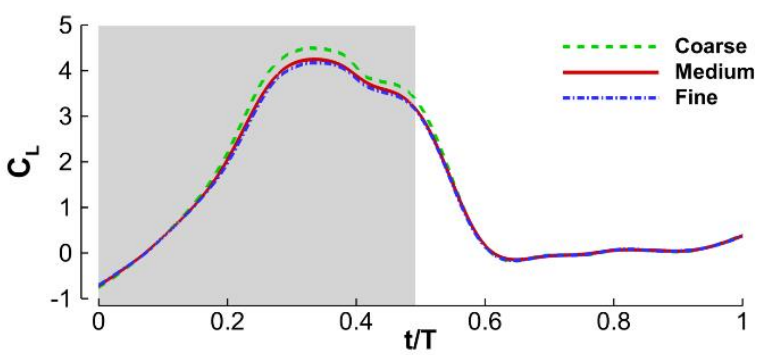

Figure 5: CFD Simulation Setup. (A)This figure shows the schematic of the computational mesh and boundary conditions employed in the current simulation for the Damselfly Flight. For ease of the display, the meshes are made less dense 4 times in each direction. (B) Grid Independent Study. The coefficient of lift is shown during the second flapping stroke. The gray shading indicates the downstroke of the forewings. Coarse $=9.8$ million; Medium=13.8 million; Fine $=15$ million. The medium grids are shown in (A).

\subsection{Results}

\subsubsection{Kinematics}

\subsubsection{A Body Kinematics}

To evaluate flight behavior during and after flight initiation, we studied the kinematics based on the reconstruction output. Figure 6A, shows the body motion of the damselfly during the takeoff stage of flight. After being stationary for some time, the wings begin to slowly peel off during the preparatory stages before initiating takeoff. The preparatory stage lasted for $30 \mathrm{~ms}$. During the preparatory stage, the insect adjusted both the stroke plane of the wings relative to the body and the pitch angle of the body. At the start of the takeoff, the body was at a pitch down orientation (about $\left.50^{\circ}\right)$. Liftoff is defined as the time duration within which the legs of the insect leave the platform, starts with the wings moving downward in a steep stroke plane. Our observation suggests that legs do not play a significant role in pushing the body up. It takes about one wingbeat for the insect to get airborne. This is a relatively short time among the insects which do not jump to initiate flight [44]. The peak 
vertical acceleration measured based on the center of mass motion during liftoff was about $3 \mathrm{~g}$. The value was less than the vertical acceleration for fruit fly takeoff (6g) [40] and butterfly takeoff (10g) [57].

The duration liftoff was about $18 \mathrm{~ms}$ and the direction of motion is up and forward while the damselfly also turns slightly. Nevertheless, the translational displacement is significantly higher than the rotational displacement. The focus of our work is on the translational displacement. The mean body Euler angles during the first wing beat are $-5.5^{\circ}, 46.6^{\circ}, 8.4^{\circ}$ and the maximum rotational velocities during the first wing beat $500 \% \mathrm{~s}$ and $200 \%$ s for roll, pitch and yaw respectively. The takeoff is slow and stable and no tumbling was observed. The body was kept straight and no body deformations were obvious. We include two subsequent flapping strokes in our analysis to document latter events during flight. During the latter strokes, the damselfly ascends, travels further and attains a more horizontal posture as flight speed increases (Figure 6C).
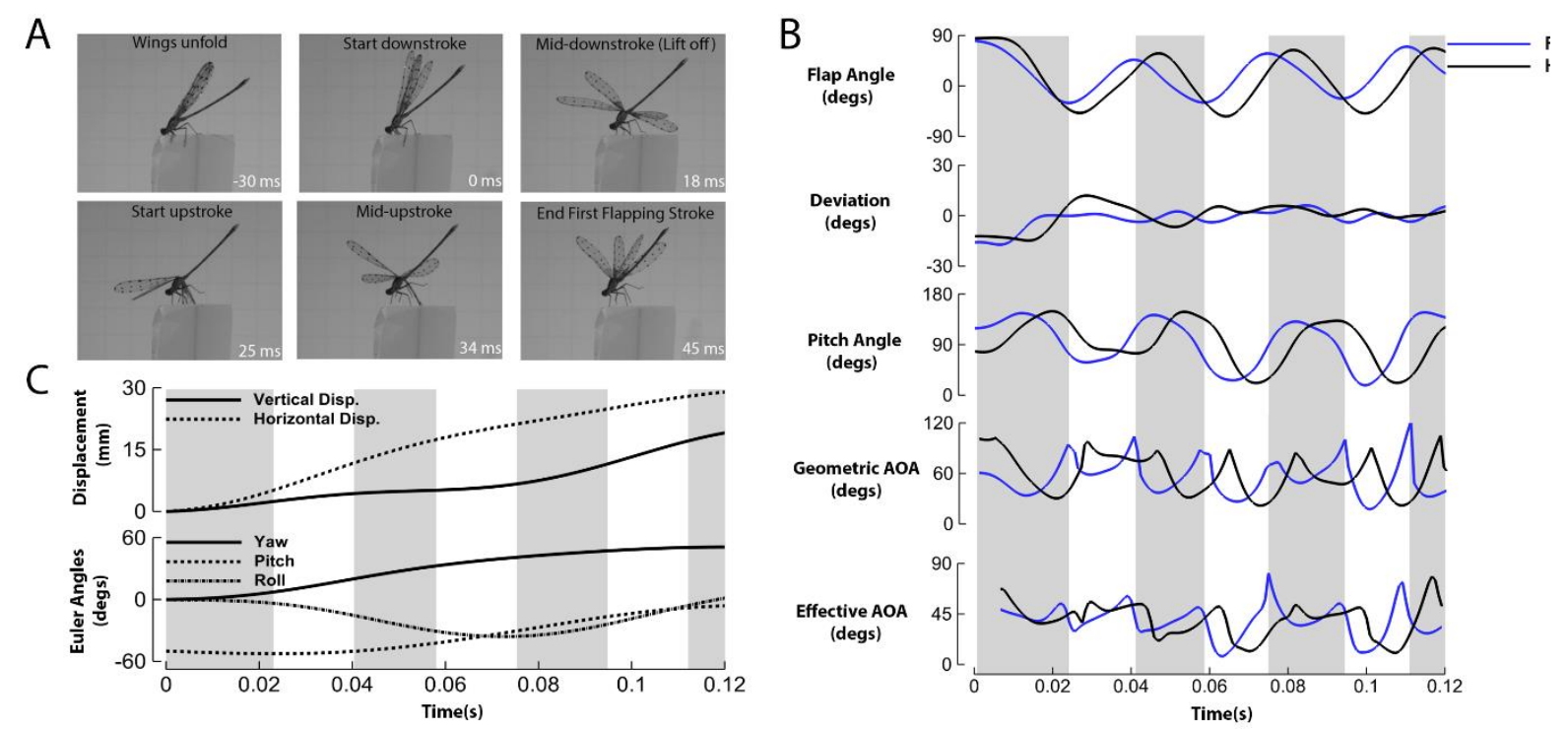

Figure 6: Kinematic events during an autonomous take-off in a damselfly (Hetaerina Americana; body weight $=0.735 \cdot \mathrm{mN}$ ). (A) A series of images depicting the sequence of events during the takeoff stroke. (B)Wing Kinematics. The kinematics are reported in the stroke plane. The definitions of the angles are in Figure 4. The angles of attack are reported at mid-span (C). Body Kinematics. The top figure shows the displament of the insect during flight. The bottom figure shows the body euler angles. 


\subsubsection{B Wing Kinematics and Deformations}

The average kinematics of the left and right wing measured in the stroke plane are shown in Figure $6 \mathrm{~B}$. The gray shading denotes the downstroke of the forewings. From the onset of flight, the motion of the fore and hind pairs of wings are offset by a slight phase difference which increases over time. The forewings lead the hind wings by about $26^{\circ}$ in the initial stroke (takeoff stroke) and about $37^{\circ}$ in the latter strokes $\left(2^{\text {nd }}\right.$ and $3^{\text {rd }}$ strokes $)$ as shown in Figure 6B.

Averaged across the three flapping strokes, the wings sweep through a stroke plane that maintains an orientation of about $18.6 \pm 5.3^{\circ}$ for the forewings and $21.6 \pm 6.1^{\circ}$ for the hindwings, measured clockwise with respect to the longitudinal axis of the body. We will refer to this as the geometric stroke plane angle $\left(\beta_{b}\right) . \beta_{b}$ is measured based on the trajectory of the wing tips. Wing kinematics including the geometric stroke plane varies from one stroke to the next, which indicates constant adjustment of the wing kinematics by the insect [67]. In the global orientation, due to steep body angle, the stroke plane with respect to the horizon $\left(\beta_{h}\right)$ is heavily influenced. The fore and hind wings flap at a stroke plane angle of $49.8 \pm 21.1^{\circ}$ and $52.8 \pm 21.4^{\circ}$, relative to the horizon, respectively, with the highest stroke plane inclination occurring in the takeoff stroke. At this instant, the stroke plane is titled steeply downwards while a less tilted stroke plane is utilized as the body becomes more horizontal in subsequent strokes. During the takeoff, $\beta_{h}$ are very similar both in up and downstroke with variations of a couple of degrees $\left(1^{\circ}\right.$ for $\mathrm{FW}$ and $7^{\circ}$ for $\left.\mathrm{HW}\right)$, whereas the difference in other strokes is more obvious wherein downstroke stroke planes are more inclined than the upstroke $\left(15^{\circ}\right.$ for FW and HW) due to a faster rate of change of body posture compared to $\beta_{b}$.

In the $1^{\text {st }}$ wing beat, the wings flap with the highest stroke amplitude recorded throughout the flight $\left(120^{\circ}\right.$ and $140^{\circ}$ for $\mathrm{FW}$ and $\mathrm{HW}$ respectively). At all times, the hindwings flap with a higher stroke amplitude (Figure 6B). As the wings flap, they are oriented at high pitch angles/AoAgeom. The instantaneous values are shown in Figure 6B. The half-stroke average AoAgeom during the $1^{\text {st }}$ downstroke is $51^{\circ}$ and $65^{\circ}$ for the fore and hindwings respectively. Whereas, the half-stroke average 
Ao $A_{\text {geom }}$ is $71^{\circ}$ and $81^{\circ}$ during the upstroke fore and hindwings respectively. During takeoff, it is evident that the hindwings flap at higher pitch both in the upstroke and downstroke. Though, in subsequent strokes, the forewings flap with higher pitch during the downstrokes (Figure 6B). In latter upstrokes, the average pitch of the fore and hindwings are similar even though the hindwings still flap with greater stroke amplitude. The upstroke $\mathrm{AoA}_{\text {geom }}$ in the takeoff stroke is about $20^{\circ}$ higher than the downstroke. Comparing the initial upstroke with latter upstrokes, the average upstroke $\mathrm{AoA}_{\text {geom }}$ is about $30-35^{\circ}$ greater. On the contrary, the stroke average downstroke $\mathrm{AoA}_{\text {geom }}$ only varies by at most $13^{\circ}$. At reversal, the wings rotate and experience the highest AoA. During this period, the wings may generate instantaneous $\mathrm{AoA}_{\text {geom }}$ in excess of $90^{\circ}$.

The effective angle of attack $\left(\alpha_{\text {eff }}\right)$ measured at $0.5 \mathrm{R}$ is reported here. The half-stroke average $\alpha_{e f f}$ during the 1 st downstroke is $44^{\circ}$ and $45^{\circ}$ for the fore and hindwings respectively. In the upstroke, half-stroke average $\alpha_{\text {eff }}$ and $45^{\circ}$ and $51^{\circ}$ for the fore and hindwings respectively. During the downstroke, the $\alpha_{\text {eff }}$ for the pair of wings is very similar, whereas, in the upstroke, slight differences are evident with the hindwings having a slightly higher $\alpha_{\text {eff }}$. Comparing the initial upstroke with latter upstrokes, the average upstroke $\alpha_{\text {eff }}$ is about $15^{\circ}$ and $23^{\circ}$ greater for the fore and hindwings respectively. On the contrary, the downstroke $\alpha_{\text {eff }}$ only varies by at most $6^{\circ}$ and $11^{\circ}$ for the fore and hindwings respectively. The effective angles of attack during this flight are higher than previously reported values. Sato et al [7] recorded a maximum $\alpha_{\text {eff }}$ of $15^{\circ}$ for majority of the downstroke during forward flight of damselflies.

The wings of damselflies are flexible, deforming and twisting as they swap through the air. Figure 7A shows the local twist distribution of the wing. Three cross sections are chosen for display; 0.5R, 0.7R, 0.9R. Similar to the wing kinematics, the twist profiles of the wings are averaged between the left and right wings. The measured twist angle across the cross-sections is at most $20^{\circ}$ and occurs at the cross-sections nearest to the wing tip $(0.9 \mathrm{R})$. The maximum twist recorded occurred during the 
mid-stroke with the forewings deforming more than the hindwings. The mid-chord of the wing $(0.5 \mathrm{R})$ has minimal twist. The AoAgeom decreases from mid-span to tip due to the twist distribution. Figure 7B shows that in the downstroke, when twist is minimal, there are minor variations AoAgeom from midspan to tip. During the upstroke, when the wing twist is more pronounced, there is more obvious variation in $\mathrm{AoA}_{\text {geom. }}$ Observing the effect of twist on the wing on the $\alpha_{\text {eff }}$, we found that the values remain relatively constant from mid-span to tip regardless of differences in AoAgeom.
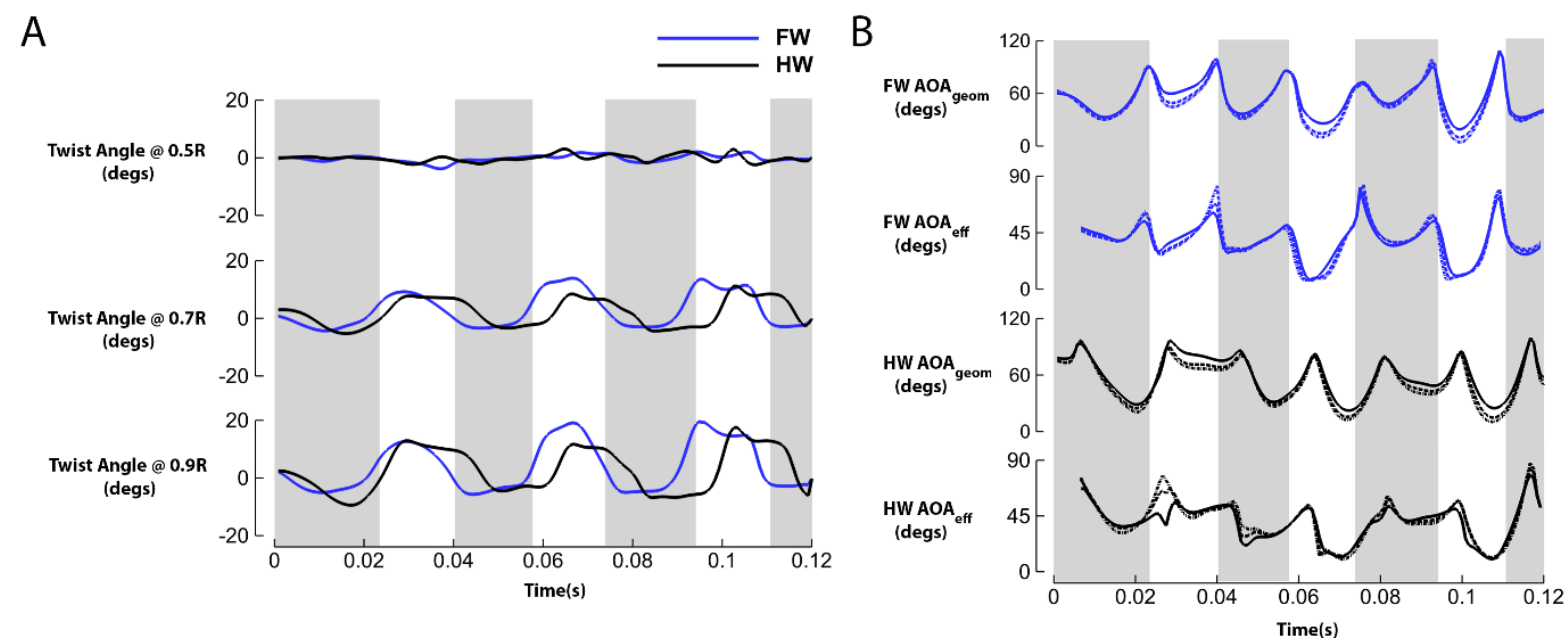

Figure 7: Local Twist and AOA Distribution from Mid-Span to Tip. (A)The local twist distribution and (B) AoA distribution at different spanwise locations $(.50 \mathrm{R}, .70 \mathrm{R}, .90 \mathrm{R})$ is reported here

\subsubsection{Identification of Vortical Structures during Takeoff Flight}

\subsubsection{A 3D Flow Structures}

Having quantified the wing motions and deformations, here, we present the three-dimensional (3D) flow phenomena based on the CFD simulation to study how the wing motions and corresponding deformations influence the aerodynamic mechanisms responsible for force generation. Figure 8 shows the evolution of the wake structures in the takeoff stroke. The start, mid and end of the downstrokes and upstrokes are shown on the left and right columns respectively. The snapshots are selected based on the timing of the hindwings. This way, we can capture the details of both pairs of wings since the forewings lead by a small phase difference. The left wings are shown. However, the same flow phenomena occur on the right wings. The vortex structures are visualized by plotting the iso-surface of 
the Q-criterion. Two iso-surface values $(\mathrm{Q}=3 \%$ and $6 \%$ of maximum $\mathrm{Q})$ are chosen to identify the wake structures. The flow structures evident on both pairs of wings are mainly characterized by tip vortices (TV) and an LEV.

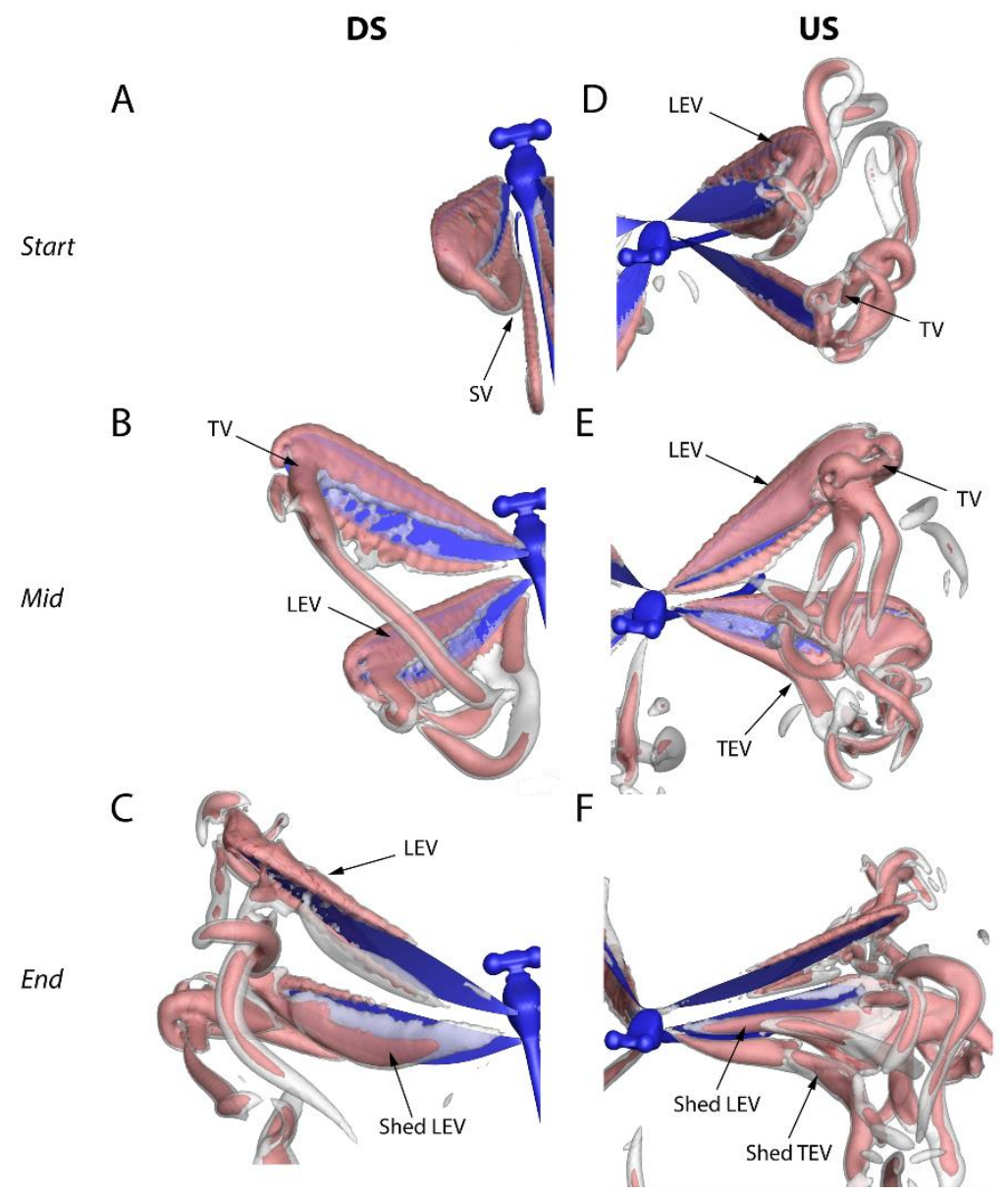

Figure 8: A time course of vortex development in the Takeoff Stroke. The vortical structures are visualized by the Q-criterion. The downstroke and upstroke are abbreviated as DS and US respectively.

At the onset of the downstroke, a starting vortex sheet (SV) sheds from the trailing edge of the wing surface. Simultaneously, an LEV is formed due to wing translation both on the fore and hindwings. The vortex rapidly grows in size and strength as the wing flaps at high AoAs. The LEV remains attached to the wing surface till wing reversal when it is shed and another LEV develops for the first upstroke only, on the anatomical lower surface of the wing which has become the aerodynamic upper surface (Figure 8 D). We do not observe multiple LEVs rather a single LEV across the wing 
surface. The excess vorticity is concentrated near the tip and sheds into a strong trailing vortex. During the upstroke, an LEV develops immediately after completion of the downstroke in the first stroke only (Figure 8C, D). The LEV actually forms first at the distal part of the wing where the combination of high wing $\mathrm{AoA}_{\text {geom }}$ and wing velocity, consequently leads to high $\alpha_{\text {eff }}$, and causes flow separation. At about mid-stroke, an LEV covering the wing surface is observed (Figure 8E). The LEV is similar in size to the first downstroke LEV (Figure 8B) at this instant. Therefore, the magnitude of circulation and thus the lift production capacity of the wing surface should be similar. The LEV remains attached to the surface and sheds during rotation. The wing-wing/wing-wake interactions are reported in another section.

\subsubsection{B Comparison of First and Subsequent Strokes.}

To understand the flow structures observed in the takeoff stroke, we compared the findings to the $2^{\text {nd }}$ stroke. Figure 9 shows selected snapshots of the evolution of the near wake in the first two wing beats. These snapshots are taken at both the fore (top row) and hindwing (bottom row) mid-strokes.

The vortex structures on the wing during the downstrokes are similar. A well attached LEV is present on the wing surface and excess vorticity feeds into a TV. The upstroke is quite different, however. In comparison to the first stroke, an LEV is not observed during the translation of the wing in the $2^{\text {nd }}$ upstroke which indicates that the wing does not dynamically stall (Figure 9C, D). However, an LEV appears for a short duration during the wing pronation due to rotation effects where the angle of attack increases rapidly as the wing rotates (Figure 9D). The appearance of the LEV is for a short duration and cannot compensate for the inability of the wing to generate substantial lift during the translational part of the stroke. Due to the absence of an LEV, no excess vorticity spirals toward the tip. The wing simply translates with attached flows on its surface which is not visible with the Q-criterion. The vortical structures around the hindwings in (Figure 9 D), are the remnants of the shed LEV during the downstroke reversal of the $2^{\text {nd }}$ stroke originating from the anatomical dorsal surface of the wing. 


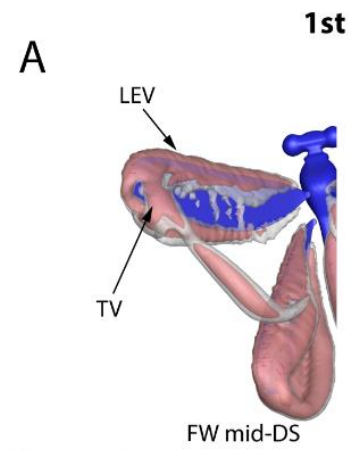

1st Stroke

B

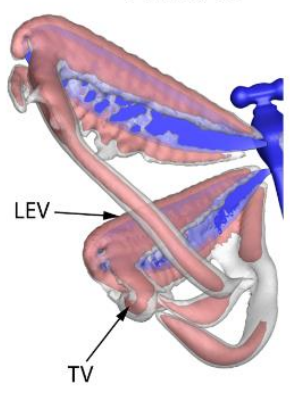

HW mid-DS

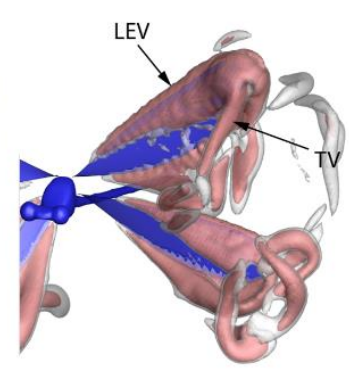

FW mid-US

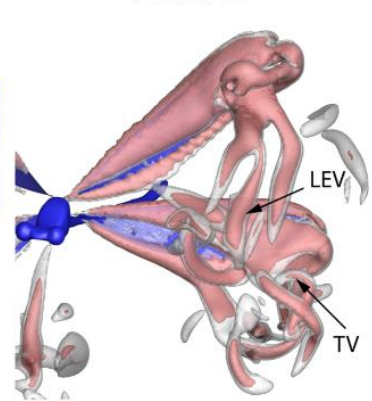

HW mid-US
C

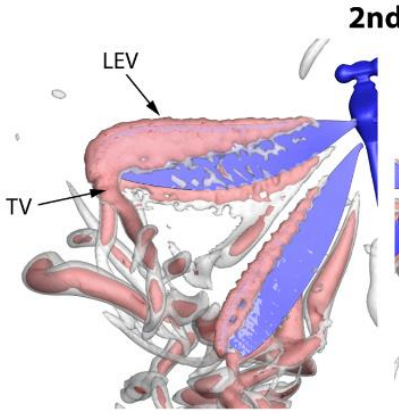

FW mid-DS

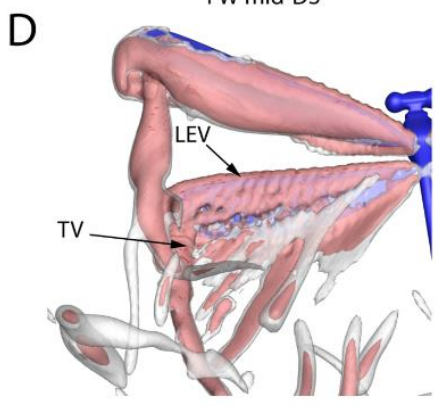

HW mid-DS 2nd Stroke

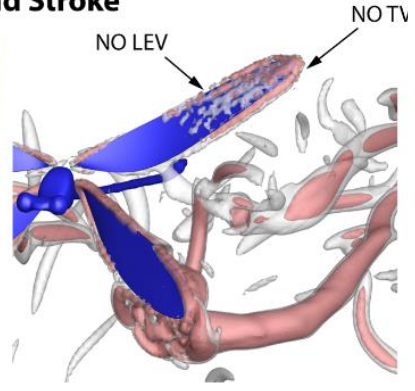

FW mid-US

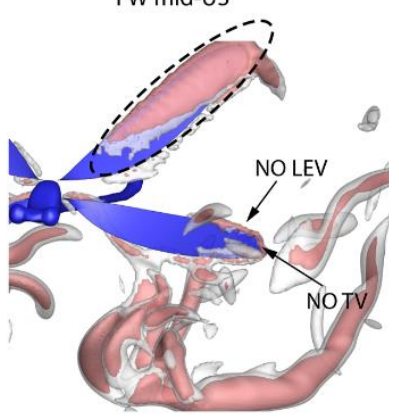

HW mid-US

Figure 9: Flow Features at mid-stroke visualized by the Q-criterion. (A) and (B) represents the flow features during the $1^{\text {st }}$ stroke at the fore and hindwing mid-strokes respectively. (C) and (D) represents the flow features during the $2^{\text {nd }}$ stroke at the fore and hindwing mid-strokes respectively. FW- Forewing, HWHindwing, TV-Tip Vortex, LEV- Leading Edge Vortex. Dashed circle in Figure 9D shows the LEV formed as the wing approaches reversal.

\subsubsection{LEV Circulation}

Having identified the vortex structures, the time history of the LEV circulation of the left wings presented in Figure 10A, is calculated at the mid-chord of the wings shown in Figure 9. The circulation is calculated by measuring the flux of the vorticity and is non-dimensionalized by $\bar{U}_{t i p}$ and $\bar{c}$. The circulation curves lag behind the wing kinematics a bit due to the delay in vortex formation at the onset of flapping. A discontinuity in the curve exists around wing reversal because of the deterioration of one vortex and the emergence of another on opposite surfaces on the wing. The circulation grows from inception of flapping, attains a maximum around the mid-stroke region and begins to attenuate as reversal approaches. This is an indication of the evolution and strength of the vortex over time. Negative LEV circulation indicates clockwise rotation of vorticity and occurs during the downstroke. The reverse happens in upstroke. 
The total circulation as well as the fore and hind wing circulation in the $1^{\text {st }}$ downstroke and upstroke are similar with the only difference being the rotational direction of the LEV. In subsequent strokes, another pattern emerges. The DS circulation is much higher than the US circulation. The hindwings, in general, have a higher circulation than the forewings over the course of the flight.

Since our method of calculating vorticity cannot distinguish between a vortex and the underlying shear layer, both on the wing surface and in between the vortices, we can attribute the circulation observed in the $2^{\text {nd }}$ and $3^{\text {rd }}$ US to the shear layer/attached flow or a very tiny and weak LEV in the subsequent upstrokes (see Figure 9C).

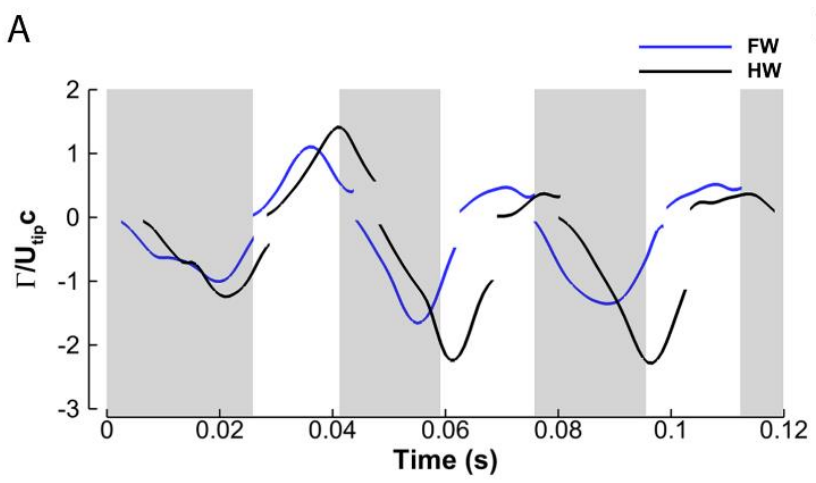

B

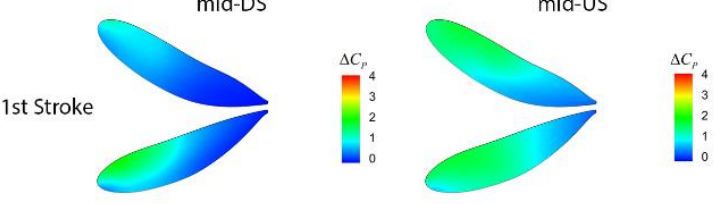

2nd Stroke
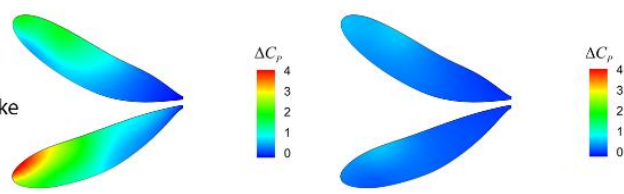

C
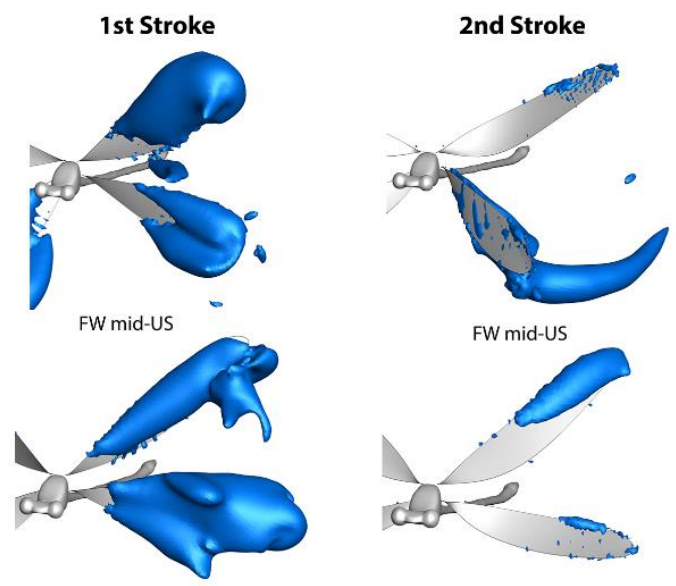

FW mid-US

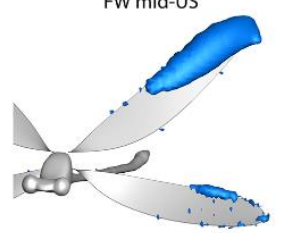

A

HW mid-US

HW mid-US

Figure 10: Quantitative measurement of Velocity and Pressure Difference on the Wing Surface. (A) Circulation at mid-Span of the left wings. (B) Pressure difference on the wing surface. (C) Isosurface of pressure coefficient $\left(C_{p}=-0.3\right)$ 


\subsubsection{Pressure Distribution and Correlation to flow structures}

To probe further into how the flow structures affect the force distribution on the wing surface, the distribution of the pressure difference between the top and bottom surface of the left wings is shown in Figure 10B. We show the pressure distributions at exactly the same snap shot as Figure 9. The pressure difference is projected onto a top view image of the wings for clarity. Moreover, the forewing mid-strokes are superimposed on the hindwing mid-strokes. The same range of contours is plotted for the $1^{\text {st }}$ and $2^{\text {nd }}$ stroke only for ease of comparison. The pressure is non-dimensionalized as $C_{p}=\left(p-p_{\infty}\right) / 0.5 \rho \bar{U}_{t i p}{ }^{2}$. The pressure difference distribution indicates what part of the wing produces the greatest velocity difference or circulation. The greatest pressure difference is generated on the distal part of the wing toward the wing tip in both US and DS. At this point, the tip speed is greatest.

When we compare the mid-upstrokes of both the $1^{\text {st }}$ and $2^{\text {nd }}$ strokes, we find drastic differences in the pressure distribution. Figure 10B, shows the pressure difference contours with the darker shade (dark blue) contours indicating regions of low pressure difference. An observation of Figure 10B indicates lower pressure difference distribution on the wing during the $2^{\text {nd }}$ upstroke as compared to the $1^{\text {st }}$ Comparing the flap velocities of the wing in these two phases of flight (see Figure 6B ), we find their velocities to be similar with the $2^{\text {nd }}$ upstroke's slightly higher. The major difference is in the pitch angle and $\alpha_{\text {eff }}$. In the second stroke, the instantaneous $\alpha_{\text {eff }}$ is as low as $7^{\circ}$ and $11^{\circ}$ for the fore and hindwings during translation, which means the flow, did not stall. Conversely, the instantaneous $\alpha_{\text {eff }}$ is as low as $29^{\circ}$ and $46^{\circ}$ for the fore and hindwings in the first upstroke, indicating flow separation at the leading edge of the wing. Figure 9, shows the presence of an LEV in the $1^{\text {st }}$ upstroke and its absence in the $2^{\text {nd }}$ upstroke. The presence of an LEV should cause a higher pressure difference between the top and bottom surface of the wing. We hypothesize that the $220 \%$ boost in the maximum pressure difference measured (1.6 in $1^{\text {st }}$ Upstroke and 0.5 in $2^{\text {nd }}$ upstroke $)$ on the wing surface is attributed to the presence of the LEV which generates a low pressure region on the wing top surface. We also showed the pressure iso-surface around the wing and found that the regions of low pressure correspond to the 
regions where the leading edge vortex was present. Consistent with the conclusion based on the isosurface of the Q-criterion as well as the pressure difference on the wing surface, we observe no low pressure region on the wing surface in the $2^{\text {nd }}$ US.

\subsubsection{Force Generation}

The damselflies in this study did not use the clap and fling to boost force production. Figure 6A shows that at rest $(-30 \mathrm{~ms})$ the wings are clasped together. However, before flight, the wings had slowly separated from each other indicating no extra vorticity to help in lift production due to peeling is present. At the end of the $1^{\text {st }}$ upstroke, the wings do not touch. The same was observed in the subsequent strokes. Since takeoff also occurred on a thin platform, ground effect is ignored. Therefore, only the flow structures and pressure distribution on and around the wing influence force generation.

The forces on the damselfly's wings were computed by the integration of the surface pressure and viscous shear stress. The force history is rendered in Figure 11A,B. FW and HW indicate the sums of the forces from the left and right forewings and hindwings respectively. For ease of visualization, the average force vectors during each half stroke are shown in Figure 11C for both the fore and hindwings. The colors of the force vectors in Figure $11 \mathrm{C}$ correspond to the lines in Figure 11A. The vectors show the relative magnitude and direction of the forces on a halfstroke basis.

During the $1^{\text {st }} \mathrm{WB}$, the insect generates substantial forces in the up and down strokes. Since the motion is up and forward, the damselfly produced significant horizontal and vertical forces as the wings flapped in a steeply inclined stroke plane. The positive lift forces, which are generated in the downstroke, supersede the negative lift generated in the upstroke. The negative lift causes vertical deceleration of the insect. The subsequent downstrokes then compensate for the negative lift support by generating larger vertical forces.

The downstroke is primarily for weight support. The maximum lift forces occurred around the mid-down stroke region with magnitudes ranging between 2-2.5 $\mathrm{mN}$ (3-3.5 times the body weight). It is during the first downstroke peak that the damselfly leaves the takeoff platform (18ms). The maximum 
lift per body mass is about $36 \mathrm{~N} / \mathrm{kg}$ which is in the measured range for Zygoptera [46]. In the subsequent strokes, the downstroke lift forces supersede the initial lift forces but generate no lift in the upstroke. However, for thrust production, it appears that both the down and upstrokes are responsible. The maximum thrust generated during the entirety of flight occurs in the first upstroke. The thrust force is as great at the lift generated in the $1^{\text {st }}$ downstroke.

In the takeoff stroke, the fore and hind wings share the burden of force generation evenly. However, in following strokes, it is obvious that the hindwings generate more force than the forewings (Figure 11B). Overall, the damselfly generates about twice the amount of total force of the $2^{\text {nd }}$ and $3^{\text {rd }}$ WB during the 1st WB indicating that the takeoff stroke was the most energetically demanding for an insect during this flight. The aerodynamic power has the same trend as the resultant force generation and peak power consumption occurs in the first stroke. However, unlike the subsequent strokes, in the initial stroke, the total forces are evenly split for both lift and thrust generation (Figure 11B). The damselfly only produces half of the total forces as of the 1st stroke in subsequent strokes with the entirety consolidated for force generation in the downstroke. This indicates that for the damselfly in this study, the upstroke is either inactive for substantial force generation $\left(2^{\text {nd }}\right.$ and $3^{\text {rd }}$ stroke) or active ( $1^{\text {st }}$ Stroke) depending on the kinematics employed during the different stages of flight. 
A

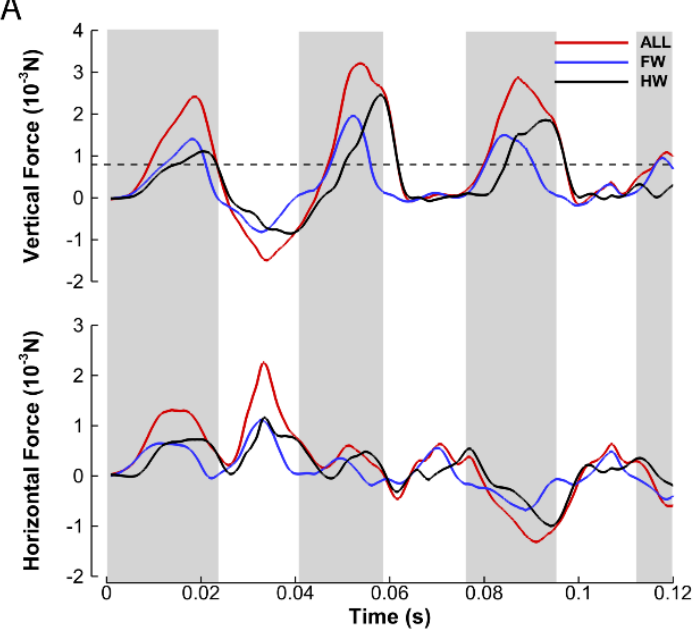

B

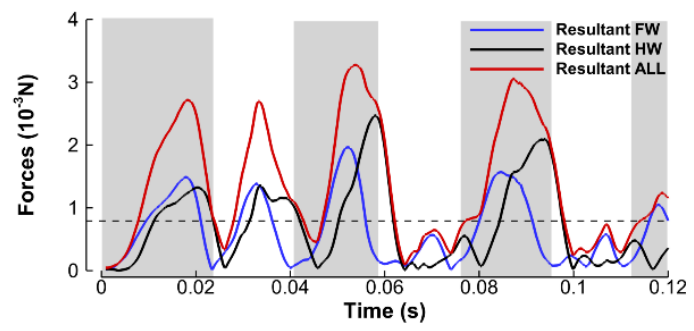

C

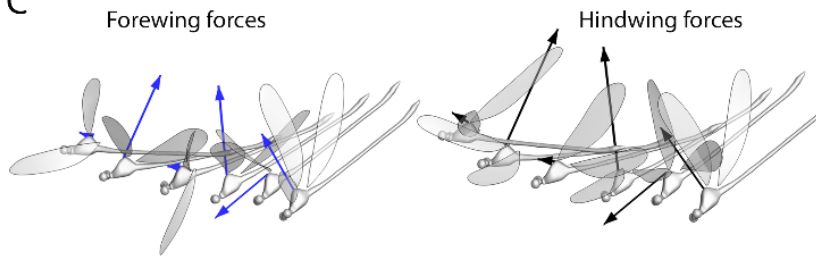

Figure 11: Force generation. (A) and (B) show the time traces of vertical and horizontal forces, and resultant forces, generated by the wings. The blue line, black line and red line represent the forces generated by the forewing, hindwings, and the sum of the fore and hind wings, respectively. (C) is a rendering of the force vector whose components are shown in (A). The colors of the vectors correspond to the line in (A).

\subsubsection{Effect of Wing-Wing Interaction (WWI) on Force generation}

Lastly, we quantify the effect of WWI. Previous studies on WWI have indicated its dependence on the phase difference between the wings [27, 68]. Proper phasing can lead to energy saving. Maximum for lift for both pairs of wings is generated when the hindwings lead by a quarter of the cycle and the distance between the wings is closest [68]. However, when the forewings lead the hindwings, the hindwing, the hindwing lift can decrease by 20-60\% [69].

To quantify the effect of WWI, we ran 3 sets of simulations; forewings only, hindwings only, and all the wings together (ALL). We ran ALL simulation with the fore and hindwings separated by real spacing between the wings of this species (Figure 3). These simulations also take into account any wake capture or wing-wake interaction effects. We observed that when there are WWI benefits on the FW, HW lift is attenuated. Most of the benefit occurred during the downstroke. During the takeoff, the FW vertical and horizontal forces were boosted by $14.3 \%$ and $12.8 \%$ respectively. On the contrary, the HW vertical and horizontal forces were attenuated by force $5.2 \%$ and $4.8 \%$ respectively. Nevertheless during the US, the HW vertical and horizontal forces were boosted by $12.9 \%$ and $10 \%$. In subsequent strokes, the lift forces were boosted by $12 \%$ ( $2^{\text {nd }}$ stroke $), 16 \%\left(3^{\text {rd }}\right.$ stroke $)$ and thrust forces were boosted 
by $4 \%\left(2^{\text {nd }} \mathrm{DS}\right)$ and $14 \%\left(3^{\text {rd }} \mathrm{DS}\right)$ on the $\mathrm{FW}$. The HW benefitted from interaction in the $2^{\text {nd }} \mathrm{DS}$, by generating a $2.4 \%$ increase in vertical force production and $10.7 \%$ increase in thrust. Whereas in the $3^{\text {rd }}$ DS, both HW vertical and horizontal forces are attenuated by $3 \%$..

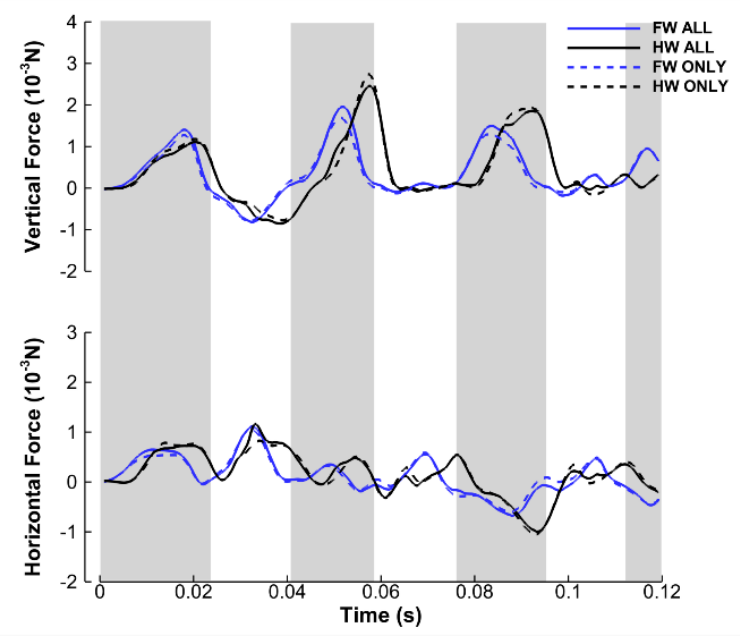

Figure 12: Effect of Wing-Wing Interaction on Force generation. The dashed lines represent the cases when the wings are isolated from each other. The solid lines represent the case where all the wings were present in the CFD simulation.

\subsection{Discussion}

Non-jumping takeoffs of damselflies are characterized by kinematics adjustments, which influence force generation. Our study indicates that while initiating flight from a steep-down body posture, the damselfly flaps its wings in steeply inclined stroke plane. The wings flap at high angles of attack and flow separates at the leading edge to form an LEV. Once the insect becomes airborne, the stroke plane becomes less inclined, and the aerodynamics of the wings change. An LEV was observed in both the DS and US during the $1^{\text {st }}$ WB where liftoff occurred. Whereas in subsequent strokes, the LEV during the US phase was absent. During the flight, majority of the forces were generated during the initial WB. Substantial forces were generated both in the US and DS. In subsequent strokes, however, substantial forces were only generated during the DS.

Here we are interested in understanding the factors led to the generation of high forces (about 3-3.5 times the body weight), equal to the total downstroke forces (Figure 11B), during the first upstroke 
of the damselfly in this study. Hence, we investigate the role of the upstroke in insect flight. Current literature indicates that the downstroke generates $80 \%$ of the total force during forward flight of Cicadas [65],50-100\% total vertical force in bumblebees as flight speed increases from 1-4 m/s [70] and 75\% of total force for damselflies in forward flight [7]. The upstroke seems to contribute minimally to force generation but could be useful in thrust generation which is usually about 10-20\% of bodyweight [25]. However, when flight mode demands an extra boost of force, the upstroke may become active such as during hovering or flight initiation.

The primary factor responsible for high forces in the US was the LEV on the wing surface (see Figure 9). This LEV was present on both the fore and hindwings contrary to previous studies on free flight $[21,56]$. The most important parameter for the formation of the vortex is the $\alpha_{\text {eff }}$ of the wing [21]. As shown in Figure 6 and reported in the results section, the AoA in the first upstroke of both pairs of wings is the highest measured throughout the flight. This creates this creates the LEV. The presence of the LEV produces the extra pressure difference on the wing as shown in Figure 10 and is responsible for amplifying the forces on the wings. We hypothesize that the LEV boosted the pressure difference in the first upstroke by as much as $220 \%$ compared to other strokes as the wings in all strokes flapped with similar velocities in the US.

The orientation of the stroke plane determines whether the pressure difference induced by the LEV will be used for lift (vertical force) or thrust (horizontal force) production. The steep body angle and the small change ( $1^{\circ}$ for FW and $\left.7^{\circ} \mathrm{HW}\right)$ in the stroke plane between the first downstroke and upstroke means that the kinematic adjustment of the orientation of the wing surface i.e. pitch, is not sufficient to redirect the aerodynamic force to generate positive lift but more suited to thrust production. In subsequent strokes, as the stroke plane inclination attenuates due to body posture, the majority of the force component is consolidated for vertical force production (Figure 11C).

Our findings indicate that in initiating flight from a steeply pitched down body orientation, the upstroke during the takeoff is critical and does not necessary play an auxiliary role to the downstroke. 
Rather, it is equally essential in ensuring a successful flight. An LEV present in the upstroke makes this possible. When the body posture becomes more horizontal, the upstroke then plays an auxiliary role to the downstroke. Therefore, depending on the flight scenario, damselflies have the ability to switch between an active and an inactive upstroke to maximize force generation.

\subsection{Acknowledgments}

I gratefully acknowledge support from National Science Foundation [grant number CEBT1313217] and Air Force Research Laboratory [grant number FA9550-12-1-007] monitored by Dr. Douglas Smith. 


\title{
Chapter 3: Flying in Reverse: Kinematics and Aerodynamics of Dragonfly (Erythemis simplicicollis) Backward Free Flight.
}

\begin{abstract}
Here, the backward flight of a dragonfly was investigated. In this flight, the insect took off from a platform and flew backward and upward for about 3 wingbeats (WB) traveling a distance of over 3 body lengths with an advance ratio of 0.41 . The dragonfly initiated flight with a body pitch angle of $87^{\circ}$ and continued to pitch up to about $95^{\circ}$ as it flew. The stroke planes of the wings were tilted backward towards the body, thus, reducing the stroke plane angle when compared to forward flight by $15^{\circ}$. Due to the global reorientation of the stroke plane owing to the vertical body posture, the aerodynamic roles of the downstroke and upstroke were switched, with the downstroke generating the propulsive force and upstroke generating the lift force. Unlike forward and hovering flight, a stronger leading edge vortex (LEV) was present during the upstroke when compared to downstroke, resulting in generation of larger aerodynamic forces in the upstroke necessary for weight support.
\end{abstract}

Keywords: backward flight, downstroke-upstroke reversal, active upstroke, low Re aerodynamics, leading edge vortex (LEV), CFD simulation

\subsection{Introduction}

Insects are capable of many aerodynamic feats including but not limited to flying forward, sideways or even hovering. Although not commonly written about, it is well known that many insects can fly backward. Hence, the concept of backward locomotion in nature is not new. Sapir and Dudley [71] recently opined that this form of locomotion could be a mechanism shared due to convergent evolution among several species because organisms such as Hummingbirds [71, 72], insectivorous birds [47, 73, 74], aquatic organisms such as anguilliform swimmers [75], electric fish [76], and terrestrial organisms such as hexapods, apart from insects have evolved to perform this type of motion with ease. For engineers and scientists, the possibilities of incorporating backward locomotion in the bid to design more robust and versatile small scale bio-mimetic vehicles seems exciting. 
Here, a dragonfly in backward flight is investigated. Backward flight is of interest because it provides an alternative in traveling from one point to another without having to reorient the body in the direction travel (Figure 13). For example, during a takeoff maneuver with the same initial body posture and intent to flight in a particular direction, a dragonfly has two major options. The dragonfly can take off from the platform, turn around and then face its final destination head-on or simply fly backward. Fully equipped with a sophisticated visual system [77] and advanced flying strategy, flying backward without having to turn around becomes a reality. To date, however, a small number of kinematics and no aerodynamic data and flow visualization are available in the literature on backward flight. The existing studies indicate that insects may use backward locomotion for predator evasion, prey capture, takeoff, station keeping and load lifting [47, 71, 78, 79].
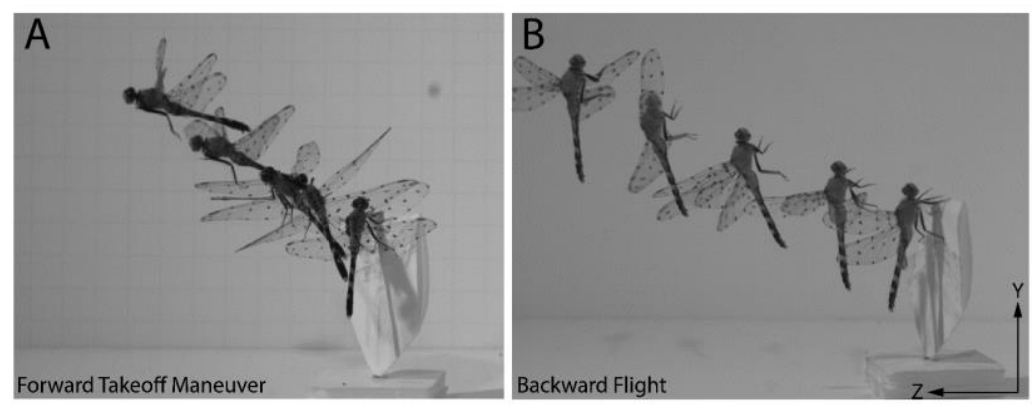

Figure 13: Forward Flight versus Backward Flight. This figure shows the two possibilities of travelling from one point to another by the same dragonfly. (A) shows a takeoff maneuver in which the insect initiates flight, turns around and heads to its destination. (B) shows a backward flight initiation which does not necessitate a maneuver .

To change flight direction, titling of the stroke plane is a popular choice used by many different flying species. To reorient the force relative to the flight path, several insect species slightly tilt their stroke plane up during backward flight. The major reorientation of the force, however, may occur due to modulation of the body posture. Mukundarajan et al. [80] observed the relationship between the body angles and the orientation of the stroke plane angles of Waterlily beetles (Galerucella nymphaeae) and its influence on their choice of flight mode. They found that a stroke plane titled upward as well as a body angle between $50-70^{\circ}$ was sufficient to induce backward flight. From an anecdotal perspective, Ellington alluded to the tilt of the stroke plane to induce retro-locomotion [25]. Large angles of attack 
have also been postulated to be employed as auxiliary mechanisms [47]. Additionally, a recent work on hummingbird flight enumerated other wing kinematics patterns employed in backward flight such as higher wingbeat frequency, higher upstroke (US) to downstroke (DS) duration ratio, higher stroke amplitudes and smaller advance ratio relative to forward flight [71].

To understand backward flight, we briefly summarize some findings from studies on hovering and forward flight of dragonflies to which we can compare our results throughout this disquisition. During forward flight, dragonflies usually flap their hindwings ahead of the forewings (counterstroking) [21, 47, 48]. In hover flight, the wings beat out of phase at about 180 degrees [28, 48]. The dragonfly beats its wings in an inclined stroke plane and generates most of the flight force in the DS $[28,49]$. The DS is conventionally regarded as lift (vertical force) producing and the upstroke thrust (horizontal force) producing $[28,47,50]$. However, the forces produced during the US are significantly less and account for about $10-20 \%$ of the body weight $[20,25]$. Body posture also differs during forward flight, hovering and backward flight. During hovering, the dragonfly body is kept horizontal [51]. In forward flight the body angle tends to be horizontal but decreases as speed increases to reduce drag [53]. On the contrary, an upright body posture $\left(100^{\circ}\right)$ was observed for a dragonfly flying backwards [47].

To generate forces, dragonflies may take advantage of high-lift mechanisms while flapping. These mechanisms include delayed stall, clap and fling, rotational circulation and wing-wake interactions (WWI). The leading edge vortex (LEV) formed during delayed stall is the most important of these mechanisms. The LEV is a region of low pressure formed on the upper part of the wing surface toward the leading edge as the wing flaps at high angles of attacks. It enhances lift by adding its circulation to the bound circulation of the wing [16]. The LEV usually stays stably attached to the wings surface and breaks down as the wing approaches the end of the stroke. So far, the presence of LEVs have been extensively reported in the downstroke phase of flight. For four-wing fliers, LEVs were observed on the forewings only. Thomas et al. [21], using smoke visualization, and Wang and Sun [27], in a computational study, reported the existence of attached flows on the hindwings of dragonflies in 
forward flight at moderate advance ratios $(\mathrm{J})$. Attached flows and the absence of LEV have been reported in upstroke phase of flight $[19,20]$. Apart from LEV formation, Wing Wing/Wing wake interactions could also be significant. In backward flight, it has been reported that WWI could boost force production as much as $15 \%$ [81].

A lack of a comprehensive discussion of backward flight of insects, therefore, serves as a catalyst in this study to improve the understanding of the kinematics, aerodynamic and flow mechanisms that make backward flight in dragonflies possible. We are interested in understanding how the motion of the wings and body is coordinated and how the flight forces are generated in this unique kind of flight. To this end, a high speed photogrammetry setup coupled with 3D surface reconstruction techniques [63] and a high fidelity Computational Fluid Dynamics (CFD) flow solver [65] were used to elucidate both the kinematics and aerodynamic features, respectively.

\subsection{Materials and Methods}

\subsubsection{Dragonflies, High speed videography and 3D surface reconstruction}

The dragonflies were captured in the grassy area of Nutter Center in Dayton, Ohio during the summer of 2012. After transporting the insects to the laboratory and cooling them down to prevent flying around in the cage and causing wing damage, their wings were dotted so that they could be tracked easily. The video footages were recorded shortly after their capture in a quiescent environment of about room temperature $\left(70^{\circ} \mathrm{F}\right)$. After warming up, the insect initiated flight without external stimulation such as prodding.

A photogrammetry setup consisting of three orthogonally arranged and synchronized cameras was used to capture the flight of the dragonfly reported in this study. After recording the free flight data, the motion was then reconstructed in Autodesk Maya (Autodesk Inc). The accuracy of the reconstruction technique as well as a thorough evaluation of the preparation methods of the insects for high speed video motion capture is identified elsewhere $[63,83]$ and just briefly outlined here. The 
methods for analyzing the free flight kinematics are also discussed therein. The morphological parameters of the dragonfly is reported in Table 2.

Table 2: Morphological Parameters for the Dragonfly in this Study. The mass and length measurement uncertainties are $\pm 1 \mathrm{mg}$ and $\pm 1 \mathrm{~mm}$ respectively.

\begin{tabular}{cccccccc}
\hline \hline Species & $\begin{array}{c}\text { Body } \\
\text { weight } \\
(\mathrm{mg})\end{array}$ & $\begin{array}{c}\text { Body } \\
\text { length } \\
(\mathrm{mm})\end{array}$ & $\begin{array}{c}\text { Forewing } \\
\text { length } \\
(\mathrm{mm})\end{array}$ & $\begin{array}{c}\text { Forewing } \\
\text { chord } \\
(\mathrm{mm})\end{array}$ & $\begin{array}{c}\text { Hindwing } \\
\text { length } \\
(\mathrm{mm})\end{array}$ & $\begin{array}{c}\text { Hindwing } \\
\text { chord } \\
(\mathrm{mm})\end{array}$ & $\begin{array}{c}\text { Flapping } \\
\text { frequency } \\
\text { (Hz) }\end{array}$ \\
\hline $\begin{array}{c}\text { Erythemis } \\
\text { simplicicollis }\end{array}$ & 130 & 40 & 34 & 8 & 31 & 10 & 27
\end{tabular}

\subsubsection{Wing Kinematics and Deformation Metrics}

The wing kinematics were measured with respect to a coordinate system fixed at the wing root (X', Y', Z'). The orientation of the wing can be described by three Euler angles; stroke angle (flap), deviation and pitch. Flapping motion is simply the redundant back and forth motion of the wing around the $\mathrm{Y}^{\prime}$ axis and is positive during the downstroke. The up and down rotations with respect to the $\mathrm{X}^{\prime}$ axis is expressed by the deviation angle as is positive when the wing rotates clockwise. The wing pitch angle is the rotation angle of the wing about the Z' axis. The effective angle of attack $\left(\alpha_{\text {eff }}\right)$ is the angle between a wing section and the oncoming flow to the wing. The oncoming flow is comprised of the vector sum of the freestream velocity and the flapping velocity of the wings. To quantify wing deformation in the torsional axis, the local twist angle is reported. A plane of least wing deformation i.e. a least-squares reference plane (LSRP) is generated based on the nodes on the reconstructed wing surface (see [63] for more discussion). Twist is the angular displacement in the torsional axis from the LSRP. These definitions are rendered in Figure 14. 


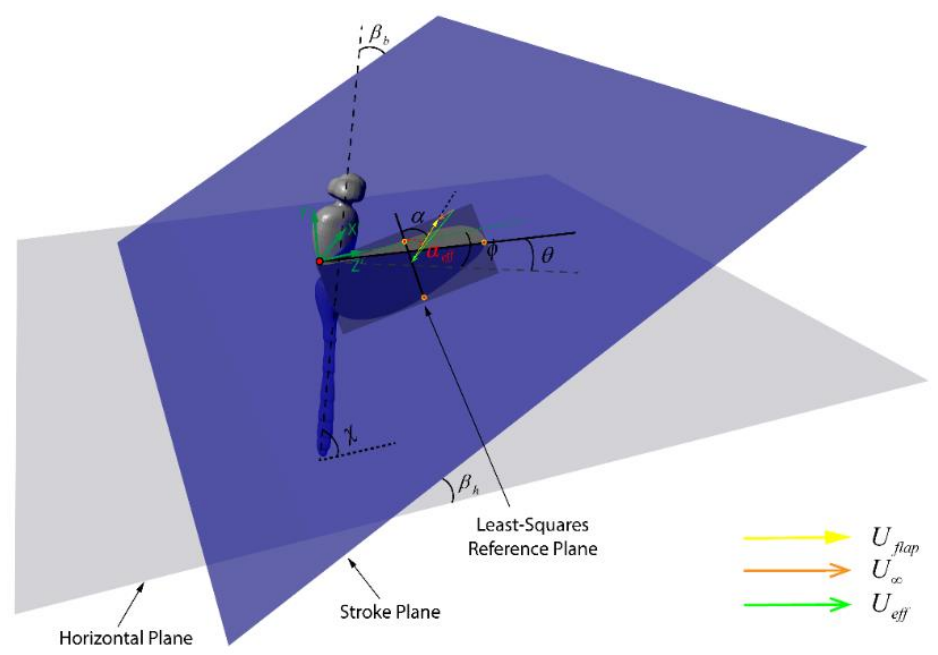

Figure 14: Kinematics Definitions. $\beta_{h}$ is the stroke plane with respect to the horizontal plane. $\beta_{b}$ is the stroke plane with respect to the longtiduinal axis of the body. $\phi, \theta, \alpha$ are the flap, pitch and deviation angles respectively. $\alpha_{\text {eff }}$ is the effective angle of attack. $\chi$ is the body attitude angle. It is the angle between the longitudinal axis of the body and the horizontal plane.

\subsubsection{CFD Simulation}

The computational fluid dynamic simulations in this study were carried out using an in-house second-order finite difference sharp interface immersed boundary method flow solver for simulating incompressible flows around 3D moving objects based on Cartesian grids. We solved the incompressible Navier-Stokes Equation (Eqn. (3)) using a finite difference method with $2^{\text {nd }}$ order accuracy in space and a $2^{\text {nd }}$ order fractional step methods for progressing in time. The convective and diffusive terms of the NS-equations were discretized using the Adams-Bashforth and implicit CrankNicolson schemes respectively. The boundary conditions were imposed by the ghost cell procedure. More details of this method and its application in other insect flight and hummingbird studies can be found in $[10,63,65,84]$.

$$
\nabla \cdot \mathbf{u}=0 ; \quad \frac{\partial \mathbf{u}}{\partial t}+\mathbf{u} \cdot \nabla \mathbf{u}=-\frac{1}{\rho} \nabla p+v \nabla^{2} \mathbf{u}
$$


The vortex structures are visualized by the $\lambda_{2}$-criterion. The simulation was run on a nonuniform Cartesian grid. The computational domain size was about $50 \bar{c} \times 50 \bar{c} \times 50 \bar{c}$ with about 14 million grid points in total. The high resolution uniform grids surround the insect in a volume of $13 \bar{c}$ $\mathrm{x} 15 \bar{c} \times 23 \bar{c}$ with a spacing of about $0.06 \bar{c}$. The stretching grids move in all three directions from the fine region to the outermost boundaries. The boundary conditions of the domain on all sides are zero gradient.

The Reynolds number for the flight is defined by $\mathrm{Re}=\frac{\bar{U}_{t i p} \bar{c}}{v}$ and is about 1480. It is measured based on the average wing tip speed $\left(\bar{U}_{t i p}=2.78 \mathrm{~m} / \mathrm{s}\right)$, mid-chord length $(\bar{c}=0.008 \mathrm{~m})$, kinematic viscosity of air at room temperature $\left(v=1.5 \times 10^{-5} \mathrm{~m}^{2} \mathrm{~s}^{-1}\right)$. The current grid set-up was chosen to ensure that the domain is sufficient and the grids are refined to ascertain grid independence of the simulation. Figure 15 shows the comparison of lift coefficient history of the second wing beat in three grids (coarse, medium and fine). The difference of both the mean value and the peak value of lift between the mediumgrid case (adopted in this paper) and the fine-grid case is less than $2 \%$. Therefore, the aerodynamic force calculations in the current study were grid-independent.

A
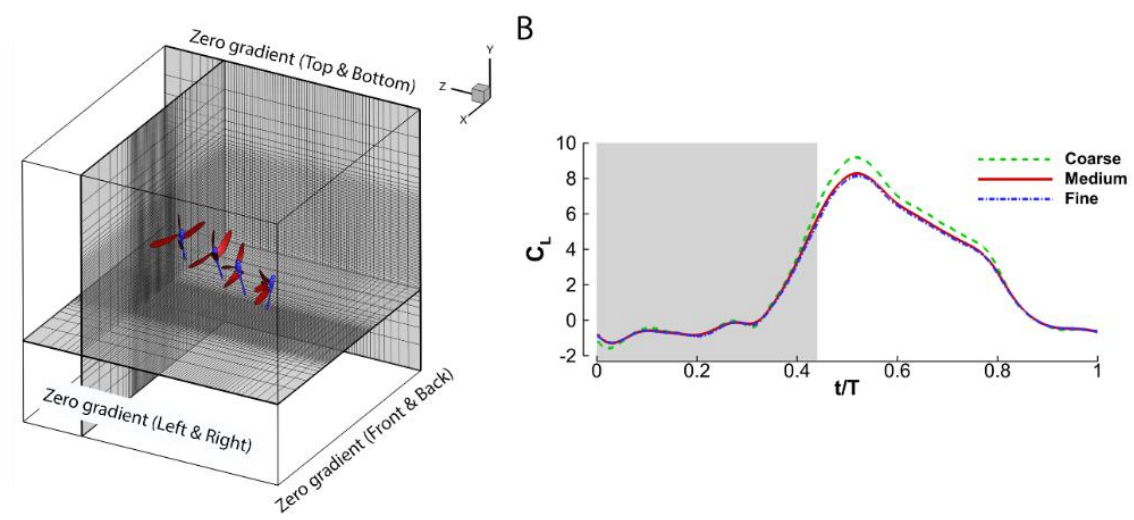

Figure 15: Computational Setup. (A) Schematic of the computational mesh and boundary conditions employed in the current simulation for the current study. For ease of the display, the meshes are made less dense 4 times in each direction. (B) Grid Independent Study. The coefficient of lift is shown during the second flapping stroke. The gray shading indicates the downstroke of the forewings. Coarse=10 million; Medium=14 million; Fine=16 million. The medium grids are shown in $(\mathrm{A})$. The gray shading is based on the downstroke. 


\subsection{Results}

\subsubsection{Kinematics}

\subsubsection{A Body Kinematics}

At the onset of flight, the dragonfly rests on a paper stool at the middle of the shooting area in a vertical posture. The initial body attitude angle is $87^{\circ}$. Figure $16 \mathrm{~B}$ shows a selected sequence of images from the high speed cameras depicting the general motion of the body. The three-dimensional (3D) reconstruction of the body motion is also shown in Figure 16C. Just as observed during maneuvers, during backward flight the dragonfly deformed its tail and this was considered in the motion reconstruction [85].The quantitative body kinematics, time histories of the body pitch angle as well as the tail angle, defined as the angle between the thorax and the tail, are all recorded in Figure 16D.

All four wings of the dragonfly as well as the tail started moving backward (upstroke) at the onset of the motion ( $\mathrm{t}=-20 \mathrm{~ms}$ ) albeit for a brief period. However, obvious body translation did not occur until the successive downstroke. This time instant is denoted as the start of the flight $(\mathrm{t}=0 \mathrm{~s})$. The insect left the platform smoothly while simultaneously pitching up (increasingly leaning backward). Both the body backward velocity and the attitude angle continued to increase within the next 2.5 flapping cycles of the forewings. Comparing the body attitude to the tail motion, it appears that the trend of changes in the tail angle are similar to the body attitude angle, with tail following the body attitude. The changes in the tail angle lag behind the changes in the body attitude angle by about half a stroke. At the beginning of the third upstroke, as the insect slowed down, it decreased its body attitude and tail angle.

The average body attitude angle was about $90^{\circ}$ during the backward flight. The wings propelled the body backward with an average velocity of about $1.1 \mathrm{~m} / \mathrm{s}$. The advance ratio, defined as the ratio of the body to the wing tip velocity is 0.41 . The center of mass of the body was also elevated about 7

chord lengths during the last two flapping cycles. However, most of the body motion occurred in the horizontal direction. 
A
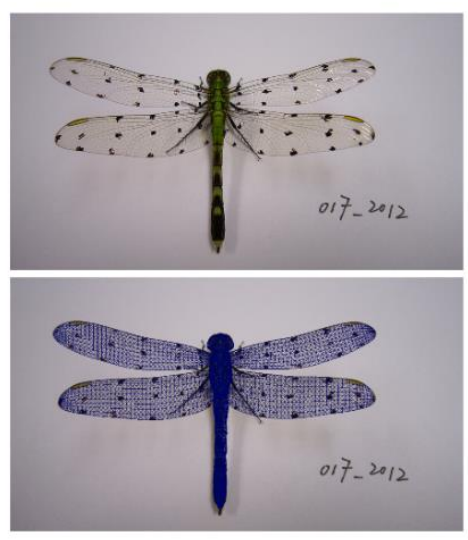

B

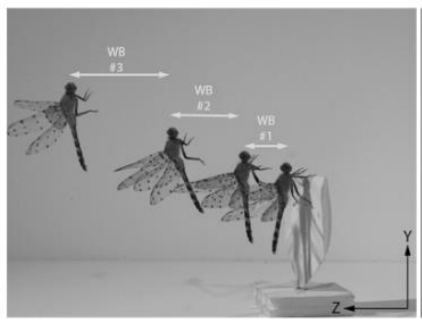

D

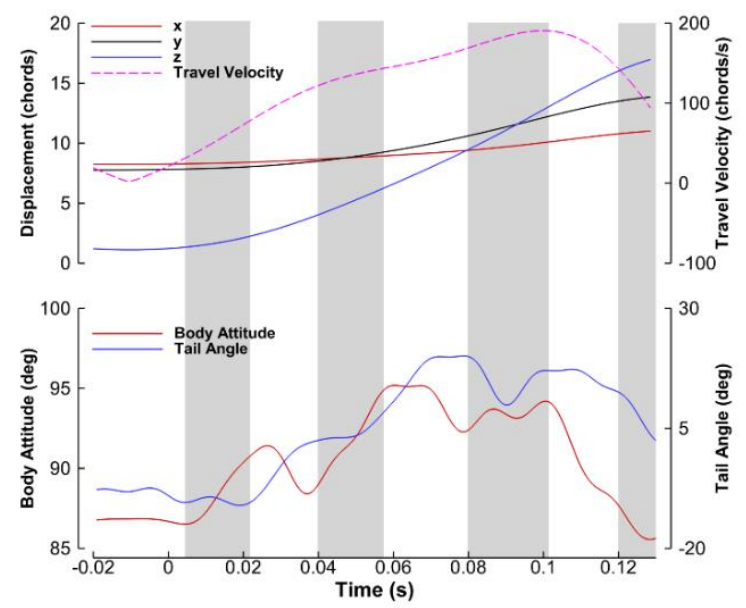

C

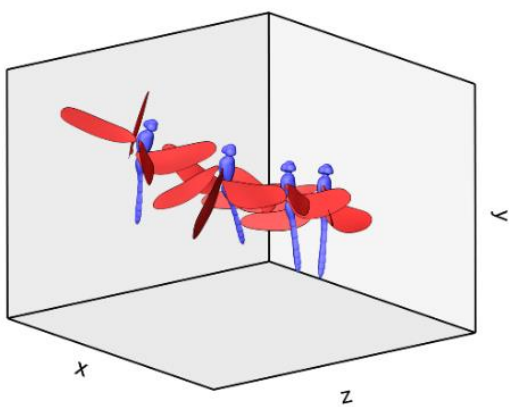

Figure 16: Body Motion During Backward Flight. (A) Orignal image reconstructed dragonfly is overlapped on the real damselfly image. The dots are the marker points placed on the wing for tracking the wing motion. (B) Snapshots (side and top views) of the dragonfly in backward flight during each wing beat. (C) 3D model of dragonfly in backward flight. (D) Body Kinematics during the flight. The gray shading here is based on the forewing timing.

\subsubsection{B Wing kinematics and Deformations}

Both wing pairs of the dragonfly sweep through a stroke plane $\left(\beta_{b}\right)$ that maintains an average orientation of about $35 \pm 5^{\circ}$, measured counterclockwise, relative to the longitudinal body axis throughout the flight. However, in the global frame, the stroke plane of the wings in backward flight is tilted upward by about $\sim 90^{\circ}$ relative to forward flight because of the large body attitude angles. This upright body posture is evident in Figure 13 and Figure 16. The tilt angle of the stroke plane relative to the horizon $\left(\beta_{h}\right)$ is $47 \pm 4^{\circ}$ with no significant difference between the forewings and hindwings.

Figure 17, shows the measured wing kinematics. The hindwings lead the forewings, but the overall flapping amplitude and velocity are similar for the fore and hind pairs of wings. The phase 
difference increases slightly from one stroke to another; $22^{\circ}, 32^{\circ}$ and $46^{\circ}$ for the first, second and third strokes respectively. Throughout the flight, the average position of the hindwings is shifted backward relative to the forewings. The phase shift may help with preventing oscillation in the body posture during flight. The ratio of the duration of US to DS changed throughout the flight increasing in the second stroke to about 1.3 and flapping back to about 1 in the third stroke.

The effective angle of attack ( $\left.\alpha_{\text {eff }}\right)$ is reported at 0.70R. Averaged across the 3 flapping strokes, the $\alpha_{\text {eff }}$ for the downstroke was $32.2 \pm 2.3^{\circ}$ and $35.1 \pm 5.8^{\circ}$, and upstroke $24.3 \pm 2.8^{\circ}$ and $26.8 \pm 5.6^{\circ}$ for the fore and hindwings respectively. These results indicate higher $\alpha_{\text {eff }}$ on the hindwings in both US and DS. These angles of attack (AoA) are high enough to form an LEV during excursion and rotation in both US and DS.

In addition to the rigid wing kinematics, we analyzed the twisting of the wing surface. The twist angle quantifies the difference between the local pitch angles of a specific chord line across the wing length compared to the LSRP. The twist angles at three spanwise locations $(0.5 R, 0.7 R, 0.9 R)$ are shown in Figure 17. The twist angle increases from mid-length to tip with hindwings showing larger twisting. The twist angles are greater for the hindwings and during the upstroke phase. Our measurements show that the twist angles could be as large as $40^{\circ}$. 

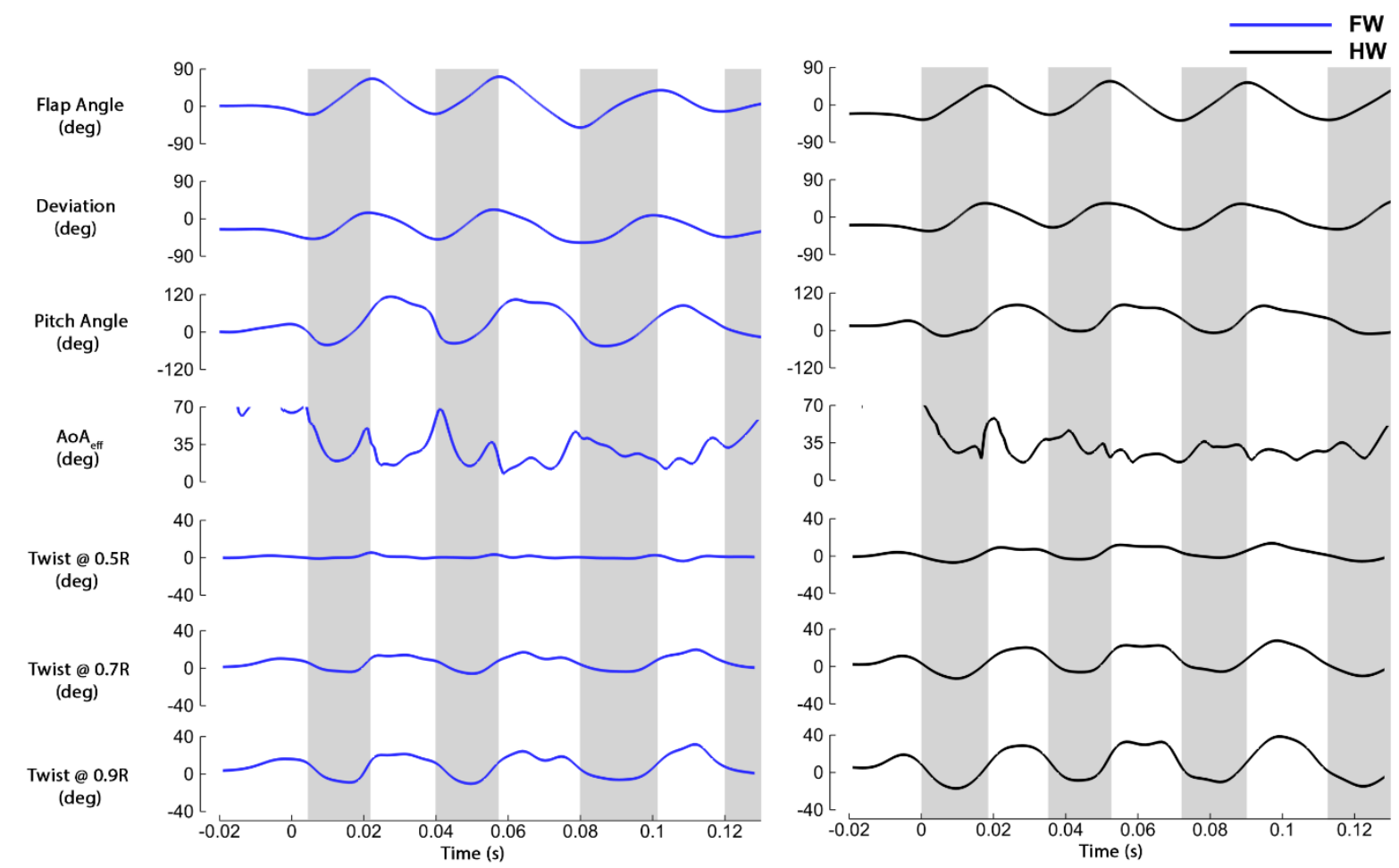

Figure 17: Wing Kinematics and Deformation Metrics. The Euler angles as well as deformation (twist) are reported here. $\alpha_{\text {eff }}$ is reported at $0.70 \mathrm{R}$. The gray shading denotes the downstroke phase.

\subsubsection{Identification of Vortical Structures during Backward Flight}

The effect of the motion and deformation of the wings is manifested in the aerodynamics footprint of the dragonfly. To visualize the 3D flow structures on and around the wing surface, we plotted the iso-surface of the $\lambda_{2}$ criterion at two different values. Here, we present snapshots of the flow field during the second flapping stroke. The snapshots are shown at the respective timings of the fore and hindwings separately for clarity since the phase difference is obvious. In addition, the flow features on the right wings are shown although the flow phenomena are the same on both sides of the wings. Most of the emphasis presented here is in the near field.

\subsubsection{A 3D Flow Structures}

As the wings flap during the downstroke, an LEV is formed due to the translation. The wing dynamically stalls during the DS. Over the course of the stroke, the LEV grows in size and strength before deteriorating as the approaches reversal. We did not observe multiple LEV's rather a single LEV 
was stably attached to the wing surface. During the DS, the flow over the fore and hindwings comprise of an LEV and TV. However, this leading edge vortex does not cover the entirety of the wing. There is no LEV around the wing base $(0-0.20 \mathrm{R})$ during the downstroke. For the rest of the wing surface, the excess vorticity in the LEV feeds into a tip vortex (TV). As the wing approaches reversal, the vortex deteriorates and is shed from the trailing edge. Simultaneously, another vortex forms on the anatomical upper surface of the wing during reversal because of the rapid increase in AoA during this period.

The LEV formed during the rotation remains on the wing during the start of the upstroke. It is quite difficult to distinguish between the LEV formed due to rotational effects or dynamic stall during wing translation. In the upstroke, the LEV formed covers the entirety of the wing surface. The LEV in the upstroke is larger in size and probably stronger than that formed during the downstroke. The tip vortex is also more pronounced and indicates the circulation strength may be greater than observed in the downstroke. The observation of the LEV in the upstroke indicates that the LEV is not limited to the downstroke phase of flapping alone. In addition, both pairs of wings (FW and HW) have an LEV on them. The flow on the FW are characterized by an LEV and TV. However, during the mid-downstroke, the HW flow consists of an LEV, TV and a trailing edge vortex (TEV) connected together to form a vortex loop. The loop will create a downward jet which will boost vertical force production. 
A

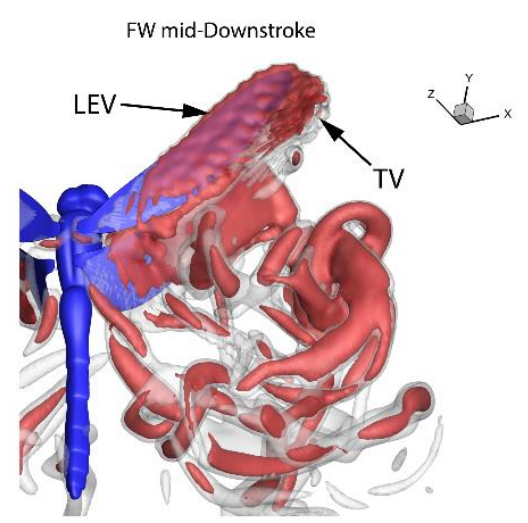

B

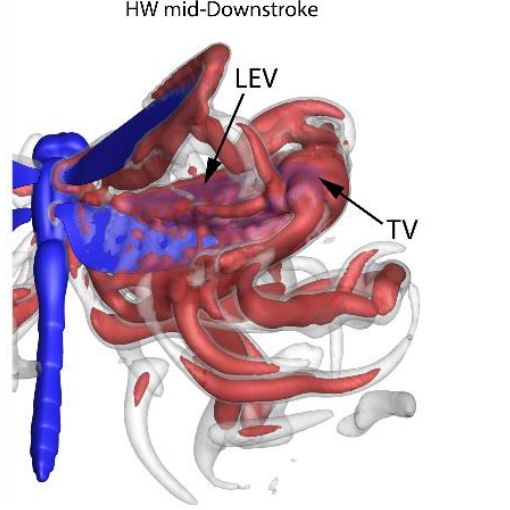

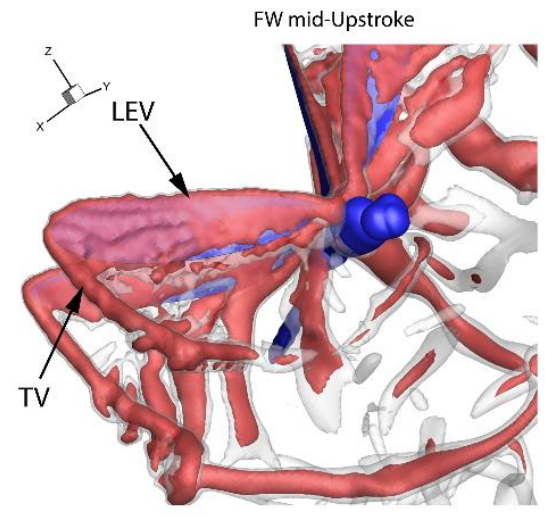

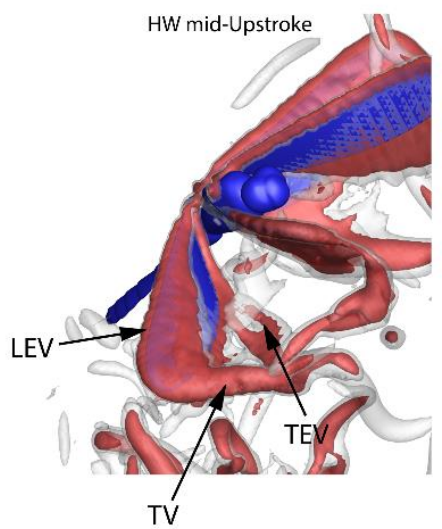

Figure 18: Flow Features at Maximum Force Production. The vortical structures are visualized by the $\lambda_{2}$ -criterion. FW- Forewing, HW-Hindwing, TV-Tip Vortex, LEV- Leading Edge Vortex, TEV- Trailing Edge Vortex.

\subsubsection{LEV Circulation}

To further probe the near field flow features of the dragonfly backward flight, we investigated the strength of the LEV throughout the second and third stroke. We measured the circulation on the wing surface due to the presence of an LEV. The circulation is calculated by taking the surface integral of the flux of the vorticity over a stroke and is non-dimensionalized by the product of $\bar{U}_{\text {tip }}$ and $\bar{c}$. The instantaneous LEV circulation at mid-chord (0.5R) is shown in Figure 19. There is a discontinuity in the curve at wing reversal because of the deterioration of the vortex and the emergence of another on opposite surfaces on the wing. Sometimes the circulation may also lag behind the kinematics due to the delay in the vortex formation process as a result of gradual growth of circulation during wing excursion. As aforementioned, the $\alpha_{\text {eff }}$ of excursion, which triggers LEV formation is greater for the 
hindwings and during the upstroke flapping phase. However, the angles of attack are high enough to induce flow separation at the leading edge.

We observe that the hindwings have larger LEV circulation values than the forewings. More importantly, the upstroke circulation is greater than the downstroke circulation. To further buttress this finding, we have included quantitative values for comparison in

Table 3. All the ratios of LEV circulation in the US to the DS are greater than unity. The spanwise distribution of circulation at maximum force production in both the $2^{\text {nd }}$ and $3^{\text {rd }}$ stroke are reported in Figure 19B and C. The circulation increases along the span and tapers toward the tip. The US circulation shown in dashed lines is much higher than the downstroke circulation. This is evident in the offset of the dashed and solid lines. Thus, further indicating the role of the upstroke in producing greater flight forces. Around the root region of the wings shown in Figure 19B and C, the US circulation values are much higher than the downstroke circulation values which corroborates the observation of the absence of the LEV around the root region in the DS visualized by the $\lambda_{2}$ criterion.
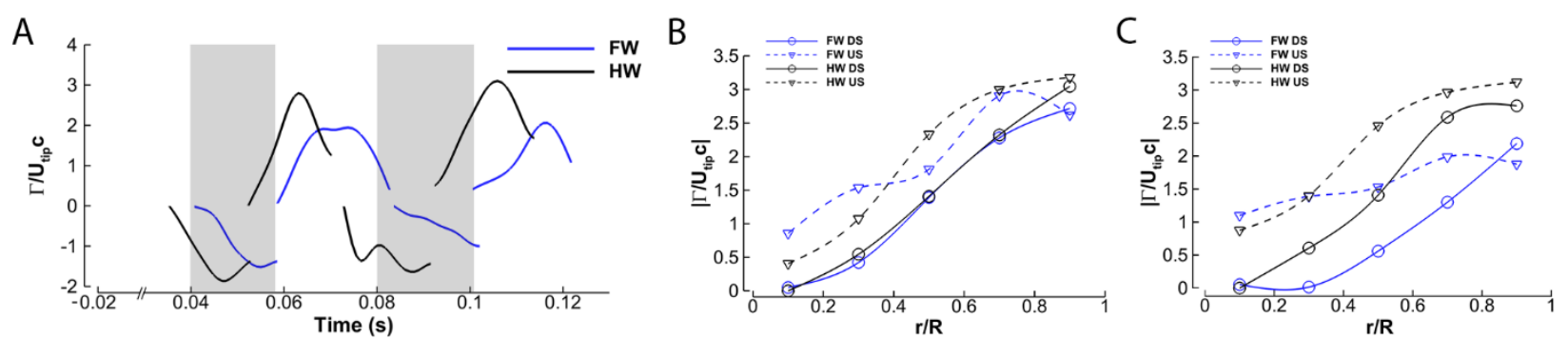

Figure 19: Leading Edge Vortex Circulation. (A) Time History of LEV during the $2^{\text {nd }}$ and $3^{\text {rd }}$ stroke. The gray shading denotes the downstroke phase for the forewings. (B)\&(C) Spanwise Distribution of LEV circulation at maximum force production during the $2^{\text {nd }}$ and $3^{\text {rd }}$ Stroke respectively. 
Table 3: Quantification of Leading Edge Vortex Circulation. Table documents the LEV circulation US to DS ratio. $\bar{\Gamma}^{*}$ represents the time halfstroke averaged values. $\Gamma_{\max }^{*}$ represents the maximum circulation per halfstroke. All values are measured at $0.50 \mathrm{R}$

\begin{tabular}{|c|c|c|c|c|c|c|}
\hline \multicolumn{3}{|c|}{ Flapping Stroke } & \multirow{2}{*}{$\begin{array}{c}\bar{\Gamma}^{*} \\
-0.94 \\
1.48\end{array}$} & \multirow{2}{*}{$\begin{array}{c}\Gamma_{\max }^{*} \\
-1.52 \\
1.89\end{array}$} & \multirow{2}{*}{$\frac{\frac{\bar{\Gamma}_{U S}}{\bar{\Gamma}_{D S}}}{1.57}$} & \multirow{2}{*}{$\frac{\frac{\Gamma_{\max _{U S}}}{\Gamma_{\max _{D S}}}}{1.24}$} \\
\hline \multirow{2}{*}{$2^{\text {nd }}$} & FW & $\begin{array}{l}\text { DS } \\
\text { US }\end{array}$ & & & & \\
\hline & HW & $\begin{array}{l}\text { DS } \\
\text { US }\end{array}$ & $\begin{array}{c}-1.36 \\
1.64\end{array}$ & $\begin{array}{r}-1.87 \\
2.80\end{array}$ & 1.21 & 1.50 \\
\hline \multirow{2}{*}{$3^{\text {rd }}$} & FW & $\begin{array}{l}\text { DS } \\
\text { US }\end{array}$ & $\begin{array}{c}-0.50 \\
1.25\end{array}$ & $\begin{array}{c}-1 \\
2.05\end{array}$ & 2.50 & 2.05 \\
\hline & HW & $\begin{array}{l}\text { DS } \\
\text { US }\end{array}$ & $\begin{array}{c}-1.26 \\
2.12\end{array}$ & $\begin{array}{c}-1.64 \\
3.1\end{array}$ & 1.68 & 1.89 \\
\hline
\end{tabular}

\subsubsection{Force Generation: Aerodynamic Force and Power}

The forces on the dragonfly's wings were computed by the integration of the surface pressure and viscous shear stress with the latter being negligible. The aerodynamic power, which is power needed to overcome air resistance, is defined as follows $p_{\text {aero }}=-\iint(\vec{\sigma} \cdot \vec{n}) \vec{u} d s$ where $\vec{\sigma}$ denotes the stress tensor, $\vec{u}$ velocity of the fluid adjacent to the wing surface, and $\overrightarrow{\mathrm{n}}$ and $d s$ the unit normal direction and the area of each element, respectively. The force and power consumption per unit muscle mass ( $\left.p_{\text {aero }}^{*}=p_{\text {aero }} / M_{m}\right)$, are rendered in Figure 20. We assumed that the muscle mass of the dragonfly is 49 percent of the body mass based on measurements by Wakeling and Ellington [52].

Consistent with the phase difference observed in the wing kinematics, the peak force generated by hindwings leads forewings force. The forewings generate maximum forces toward the end of the stroke whereas the hindwings do so at about midstroke. The magnitude of peak vertical forces generated by fore and hind wings are very similar in downstroke, while the peak vertical force generated by hindwings is about twice larger than that of forewings in the $2^{\text {nd }}$ and $3^{\text {rd }}$ strokes. The peak horizontal force for the fore and hindwings are very similar although on average the hindwings still generate larger horizontal forces. 
Majority of the vertical force is generated during the upstroke, while the force generated in the downstroke mostly propels the body in the direction of motion. Both fore and hind pairs of wings generate larger force in upstroke when compared to downstroke. This is particularly evident for hindwings, resulting in significantly larger normal force being generated by hindwings when compared to forewings. The peak vertical and horizontal forces during the flight are about 9 and 5.5 times the body weight respectively. Throughout the stroke, the dragonfly generates an average vertical force 2.53 times the body weight to sustain flight while propelling backward with an average force of 1.5 times the body weight. During flight, the forewings generate similar amounts of vertical force as the hindwings in the $1^{\text {st }}$ stroke, whereas to induce rapid ascent, the hindwings almost double their force production in the $2^{\text {nd }}$ and $3^{\text {rd }}$ stoke. The average power consumed by the dragonfly throughout the flight was $145 \mathrm{~W} / \mathrm{kg}$. Overall, this was in the same range $(76-156 \mathrm{~W} / \mathrm{kg}$ and $160 \mathrm{~W} / \mathrm{kg}$ ) measured by Wakeling and Ellington [52] measured by Azuma et al [50], respectively.
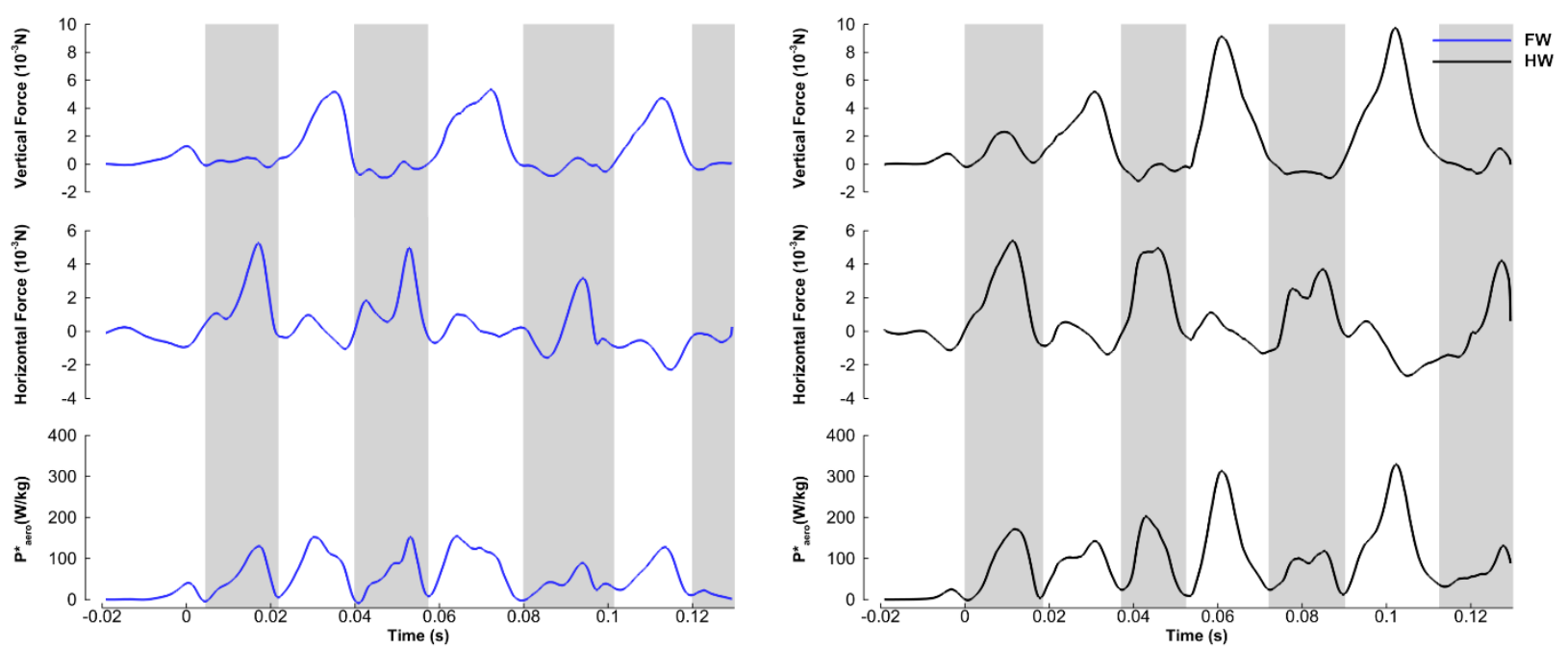

Figure 20: Force Generation and Power Consumption. The aerodynamic forces and aerodynamic power consumption are reported here. The gray shading denotes the downstroke phase.

\subsection{Discussion}

Backward flight in dragonflies is characterized by distinct alterations in kinematics and aerodynamic features which are different from both hovering and forward flight. Our study indicates 
that dragonflies are able to use backward flight as alternative to having to first reorient the body before flying in a desired direction (see Figure 13). Backward flight is not simply a transient behavior but can be sustained for a long period of time (at least 3-4 wing beats in this case) with a flight speed of 1.1 $\mathrm{m} / \mathrm{s}$. To do so, tilting of the stroke plane towards the body by about $15^{\circ}$, coupled with tilting of the stroke plane with respect to the horizontal while maintaining a steeper body posture is necessary. Aerodynamically, we also noted that majority of the forces were generated during the upstroke flapping phase compared to the downstroke. A stronger LEV in the US was responsible to greater force production. These findings were corroborated by the flow visualizations in Figure 18.

Here, we compare our findings, that is, kinematic, aerodynamics and flow physics with hovering and forward flights, which have been extensively documented in literature. A summary of previous literature on different flight modes is shown in Table 4 . The relatively high body angles $(\chi)$ during dragonfly backward flight parallels the same observations as reported in hummingbird and insect backward flight and could be a mechanism of convergent evolution. However, $\chi$ was significantly larger than observations in hummingbirds $\left(50-75^{\circ}\right)$, which utilize a horizontal stroke plane as well as in the flight of waterlily beetles $\left(50-70^{\circ}\right)$, which utilize an inclined stroke plane just like dragonflies [71, 80]. Although steep body posture has been posed to generate higher drag in Hummingbirds due to their wide body resulting in a higher projected area, it is not certain whether dragonflies rely on their body drag due to slenderness of their bodies. While body drag may be present, it is estimated to be about 20 times less when compared to the aerodynamic forces being generated by the wings. 
Table 4: Kinematic Parameters of Several Insects in Flight.

\begin{tabular}{|c|c|c|c|c|c|c|c|c|c|c|c|}
\hline \multirow{2}{*}{ Animal } & \multirow{2}{*}{$\begin{array}{l}\text { Flight } \\
\text { mode }\end{array}$} & \multirow{2}{*}{$\operatorname{Re}$} & \multirow{2}{*}{$J$} & \multirow{2}{*}{$\begin{array}{c}\text { Phase } \\
\text { shift } \\
\left({ }^{\circ}\right)\end{array}$} & \multicolumn{2}{|c|}{ Stroke plane $\left({ }^{\circ}\right)$} & \multirow{2}{*}{$\begin{array}{c}\text { Body } \\
\text { Angle } \\
\left(^{\circ}\right) \\
\chi\end{array}$} & \multicolumn{4}{|c|}{ Effective Angle of attack $\left({ }^{\circ}\right)$} \\
\hline & & & & & $\beta_{\mathrm{f}}$ & $\beta_{\mathrm{h}}$ & & $\alpha_{f}^{\text {down }}$ & $\alpha_{\mathrm{f}}^{\text {up }}$ & $\alpha_{h}^{\text {down }}$ & $\alpha_{h}^{\text {up }}$ \\
\hline \multirow{6}{*}{ Dragonfly } & $\begin{array}{c}\text { Tethered } \\
\text { Hovering } \\
{[86]}\end{array}$ & 4232 & 0 & 22 & 53 & 44 & 14 & 83 & 10 & 87 & 15 \\
\hline & $\begin{array}{c}\text { Hovering } \\
{[51,87]}\end{array}$ & 1350 & 0 & 180 & 52 & 52 & -- & -- & -- & -- & -- \\
\hline & $\begin{array}{c}\text { Forward } \\
\text { [27] }\end{array}$ & 3100 & 0.30 & 60 & 52 & 52 & -- & -- & -- & -- & -- \\
\hline & $\begin{array}{c}\text { Forward } \\
\text { Ascending } \\
{[50]}\end{array}$ & 3200 & 0.13 & 77 & 37 & 40 & 10 & 24.1 & 11.8 & 27 & 22.9 \\
\hline & $\begin{array}{c}\text { Forward } \\
\text { Ascending } \\
{[88]}\end{array}$ & -- & $0.21-0.47$ & $47-110$ & $9-26$ & $7-29$ & $\begin{array}{l}22.6- \\
35.7\end{array}$ & -- & -- & -- & -- \\
\hline & $\begin{array}{l}\text { Backward } \\
\text { (current) }\end{array}$ & 1135 & 0.41 & $22-46$ & 47 & 47 & $85-95$ & 23.6 & 31.5 & 31.8 & 41 \\
\hline Hummingbird & $\begin{array}{c}\text { Backward } \\
{[71]}\end{array}$ & -- & 0.3 & -- & \multicolumn{2}{|c|}{$0-6$} & $50-75$ & -- & -- & -- & -- \\
\hline $\begin{array}{l}\text { Waterlily } \\
\text { Beetle }\end{array}$ & $\begin{array}{c}\text { Backward } \\
{[80]}\end{array}$ & -- & -- & -- & \multicolumn{2}{|c|}{$0-30$} & $50-70$ & -- & -- & -- & -- \\
\hline
\end{tabular}

$R e$, Reynolds number; $J$, advance ratio; $\beta_{\mathrm{f}}$ stroke plane of forewing; $\beta_{\mathrm{h}}$ stroke plane of hindwing; $\alpha_{\mathrm{f}}^{\text {down }}$, instantaneous effective angle of attack of forewing at mid-downstroke; $\alpha_{\mathrm{f}}^{\text {up }}$, instantaneous effective AoA of forewing at mid-upstroke; $\alpha_{\mathrm{h}}^{\text {down }}$, instantaneous effective AoA of hindwing at mid-downstroke; $\alpha_{\mathrm{h}}^{\text {up }}$, instantaneous effective AoA of hindwing at mid-upstroke.

With regard to the wing kinematics, during backward flight, the dragonfly swept through a stoke plane inclined at $35 \pm 5^{\circ}$ respect to the longitudinal axis of its body. This angle is shallower than the stroke plane angle in forward flight of dragonflies by only $15^{\circ}[50,67]$. The stroke plane with respect to the horizon during backward flight was reported as $47 \pm 4^{\circ}$ for both pairs of wings. Compared to the forward flight of dragonflies of similar mass and morphology [67], the stroke plane with respect to the horizon during backward flight is about $20-40^{\circ}$ greater. Compared to Norberg's [51] data on hovering, $\beta_{\mathrm{h}}$ used by this dragonfly in backward flight was about $15^{\circ}$ less.

Based on the kinematics employed during backward flight, we ran CFD simulations to understand the flow physics around the wings. The flow over the wing surface was characterized by a strong LEV due to flow separation. The LEV was present on both fore and hind pairs of wings. Contrary 
to previous works on dragonfly forward flight, the presence of the LEV was not limited to the FW alone but was also evident on the HW $[21,27,56]$. The LEV was also present in the US and DS. However, the LEV in the US was stronger than the DS's. We verified this finding by measuring the leading edge vortex circulation of the wing and found US:DS LEV circulation ratios as high as 2.5 and 1.7 for the fore and hindwings respectively. On the contrary, in previous works on the free flight of insects and birds, it was reported that the circulation of the leading edge vortex is significantly larger in downstroke when compared to upstroke $[19,20,89,90]$. Unlike the DS, many studies have reported the absence of an LEV during the upstroke. Willmott et al [20], showed based on smoke visualization of hawkmoth forward flight, no hints of an LEV to enhance lift during the upstroke. Thomas et al [21] also demonstrated this fact with smoke visualizations of dragonflies in tethered and free flight. The reason for LEV absence is that during the upstroke, the wing slices through the air like a knife, resulting in very low effective angles of attack. Wang and Russell [28] measured these angles to be as low as $8^{\circ}$ during hovering flight of dragonflies. Even under steady conditions, an airfoil will not stall at this angle. Hence the absence of an LEV during the upstroke. Wang and Sun [27], using computational simulations also verified the absence of the LEV in the upstroke in hovering as well as forward flight of dragonflies ( $\mathrm{J}=0-0.6)$. In backward flight, on both pairs of wings, the average $\alpha_{e f f}$ at $0.70 \mathrm{R}$ during the downstroke was $42.5 \pm 3.1^{\circ}$ and $48.4 \pm 3.9^{\circ}$, and upstroke $44.3 \pm 4.5^{\circ}$ and $54.13 \pm 4.5^{\circ}$ for the fore and hindwings respectively. These $\alpha_{e f f}$ are sufficient to form a stably attached LEV over the wing. 


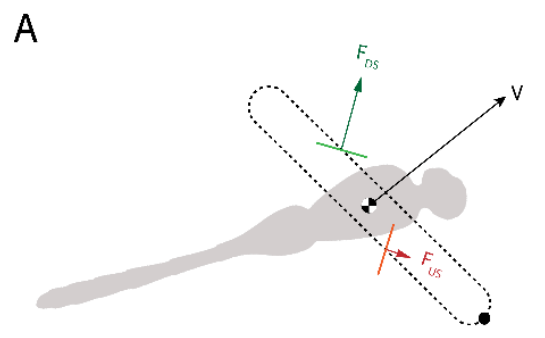

Forward Flight
B

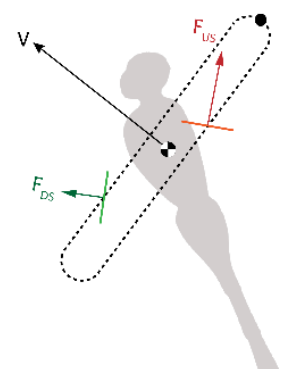

Backward Flight

Figure 21: Illustration of Forward Flight Versus Backward Flight. The length of the arrows denote the downstroke (green arrow) and upstroke (red arrow) forces based on their relative importance during (A) forward flight and (B) backward flight. The dashed lines indicate the inclined stroke plane. The black dot is placed at the bottom of the stroke plane in forward flight. By simply rotating the body to an upright posture, the black dot changes position to show the effect of body posture on rotation the stroke plane.

The presence of the LEV during the US and DS indicates substantial force production during both half strokes. LEV circulation results also indicate that the US will produce larger aerodynamic forces than the downstroke. Hence, during backward flight, we have evidence of a very aerodynamically active upstroke. Current literature reports that the downstroke generates $80 \%$ of the total force during forward flight of Cicadas [65], 75\% of total force for damselflies in forward flight [7], 50-100\% total vertical force in bumblebees as flight speed increases from 1-4 m/s [70] and vertical forces which are $80 \%$ of body weight in Hawkmoths (Manduca sexta). The upstroke when used is usually useful in horizontal force generation which is usually about $10-20 \%$ of bodyweight [20, 25]. As flight speed increases, the relative contribution of the upstroke in force production may also decrease[25]. To the authors' best knowledge, the only evidence of the upstroke producing larger forces for weight support was in the hovering flight of Drosophila [26].

A graphical representation of force production in forward and backward flight is rendered in Figure 21. An obvious difference is the body angle between forward and backward flight. At moderate advance ratios, the downstroke generates majority of the force for weight support. This is well established in literature. However, because of the steep body posture in backward flight, by rotating the body during forward flight by $90^{\circ}$ to form an upright body posture, the aerodynamic roles of the upstroke and downstroke are reversed. This is evident in Figure 20 wherein during the backward flight, 
the vertical forces are generated during the upstroke and horizontal forces in the DS. The vertical forces on average can sustain the weight of the dragonfly by $2.5-3$ times, much more than $10-20 \%$ of body weight which the US is capable of producing in other flight modes. To ensure and sustain backward flight, the role of the upstroke in generating substantial flight forces cannot be overemphasized.

\title{
3.5 Acknowledgements
}

I gratefully acknowledge support from National Science Foundation [grant number CEBT1313217] and Air Force Research Laboratory [grant number FA9550-12-1-007] monitored by Dr. Douglas Smith.

\section{Chapter 4: Optimized Body Deformation in Dragonfly Maneuvers}

\begin{abstract}
Tail bending associated with maneuvering flight of insects is a known phenomenon although there are only a few studies which analyze and quantify the effects and benefits of body configuration changes. We hypothesized that these configuration changes help reduce the energy expenditure in flight. This is quantified by the magnitude of the aerodynamic torque generated by the insect during the maneuver. To test our hypothesis, a dragonfly body model was constructed with the ability to bend at the joint between the thorax and the tail. An optimization problem was defined to find the dynamic body configuration which minimizes the total pitch and yaw torque. The magnitude of the tail deflection was found to be directly correlated with the yaw velocity of the body. Most importantly, our results indicate that for executing the same aerial maneuver, an insect with a flexible body was found to require substantially smaller flight torque when compared to an insect with identical morphology but possessing a rigid body. In addition, changes in the instantaneous mass distribution of the body had the most substantial effect on reducing the flight torque, while the inertial term due to the tail movement had a smaller effect.
\end{abstract}




\section{Nomenclature}

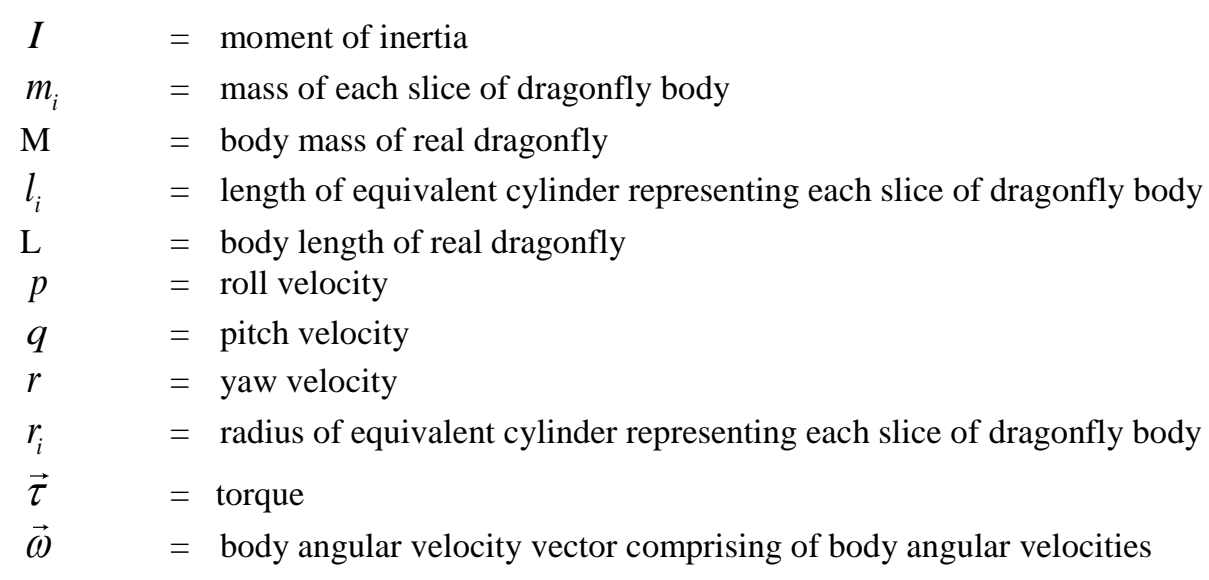

\subsection{Introduction}

To change flight heading, insects perform a variety of aerial maneuvers. In many maneuvering flights such as predatory or evasive flights, the dynamics of the motion is as important as the final change in the flight orientation. This may be due to several reasons, such as a desired flight path or considerations like the energetic cost of the flight. Therefore, in such situations, the required flight torque is dictated by the body motion as well as its morphology such as mass and moment of inertia neglecting the limitations due to the insects control system and sensory equipment etc. If the body is rigid, the only source of torque generation is the wing which generates aerodynamic force by moving back and forth through the air. Changes in the wing kinematics are the most important means by which an insect alters the flight [91]. However, if the body is capable of deforming as shown in Fig. 1, changes in the mass distribution can affect the motion by two means; first by generating inertial torque associated with the rate of change of the body moment of inertia and second by changing the response of the body to the flight torque by varying the instantaneous distribution of the mass. In the majority of studies on insect flight dynamics, the rigid body assumption is used in study to simplify the analysis $[92,93]$. However, this assumption imposes several restrictions on the problem such as aforementioned. Although the existence of abdominal deformation, i.e. flexion and deflection, in insects such as locusts and fruit flies has been known for decades $[30,94,95]$, the extent to which the flexibility of the body affects the flight is not clear yet. Scientists and engineers have inferred from observations that insects 
may bend their bodies to decrease the moment of inertia about the axis of rotation and to dampen the effects of perturbations for stability purposes using their abdomen as a control surfaces [96]. Abdominal deflection is also employed as a strategy to create asymmetric body drag profiles to increase or decrease rotational inertia. In essence, maneuvers are not restricted only to asymmetric wing kinematics changes[97] but they employ a more complicated approach to increase efficacy and efficiency of their flight.
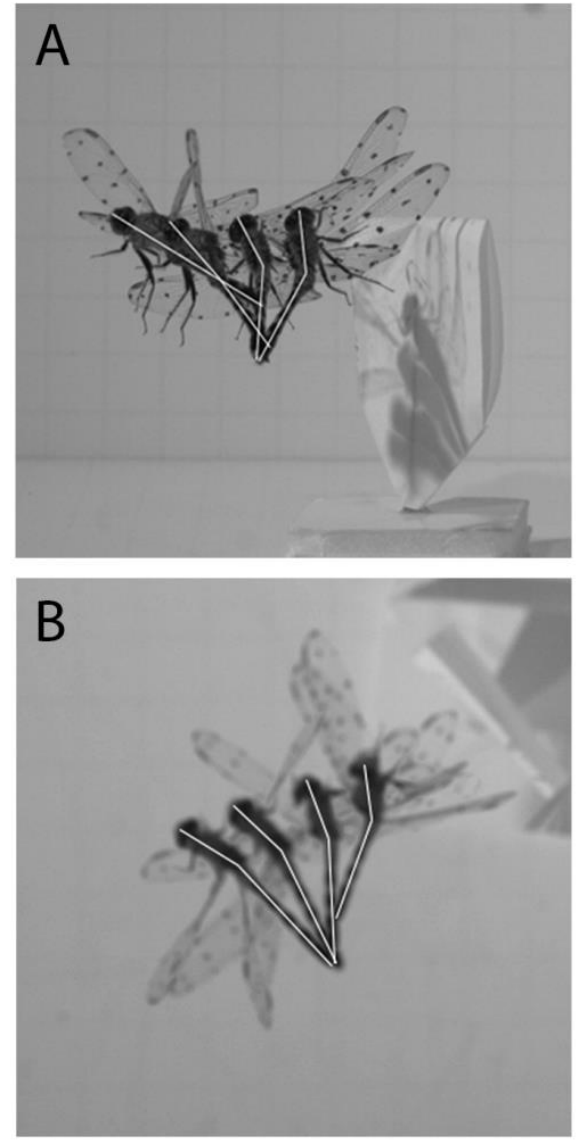

Figure 22: High Speed Photograph Indicating Tail Bending in Dragonflies. A selected sequence of images of a dragonfly in free flight is shown. (A) and (B) are images taken by the forward facing and downward facing cameras, respectively. The orientations of the thorax and the tail are indicated by white lines for clarity.
In this study, we focus on how body deformation affects the dynamics of motion. To enumerate the effects of moment of inertia changes due to body deformation on the dynamics of flight during a maneuver, we built a flexible body model and developed computer codes to accurately calculate the moment of inertia while taking into account body flexibility effects. To understand the physics behind body deflection, we hypothesized that the tail of a dragonfly deflects dynamically to minimize the average total torque during a maneuver. Here, we developed an optimization problem to carry out the investigation. 


\subsection{Materials and Methods}

\subsubsection{Calculation of Moment of Inertia}

Moment of inertia (MOI) is a measure of the resistance of a body to rotation and because insect body morphologies are not comprised of regular geometric shapes, their body shapes are approximated in order to calculate the MOI [98-102]. On the contrary, in this paper, we accurately calculate the moment of inertia tensor with the confidence of reliability in all degrees of freedom. The moment of inertia for the dragonfly was obtained from a 3D model constructed in MAYA (Autodesk, San Rafael, CA, USA) based on images of a common species; Erythemis simplicicollis, found in North America. Two images; a top and side view are necessary for accurately constructing the 3D model. The nondimensional body morphological data obtained from the dragonfly model is included in Table 1 . The MOI data is non-dimensionalized in Table 5 by $\mathrm{M} / \mathrm{L}^{2}$, where $\mathrm{M}$ is the body mass $(252.52 \mathrm{mg})$ and $\mathrm{L}$ is the body length $(43.95 \mathrm{~mm})$.

For analysis, the 3D model was sectioned into 21 pieces as shown in Fig. 2. Slices 1-3, 4-7 and 8-21 make up the head, thorax and tail regions respectively. We established that 21 slices provided enough accuracy for our study. However, since a computer algorithm is responsible for the calculation of MOI, the number of sections can be infinitely increased and the thickness of each section need not be uniform. 
Table 5: Geometric Data of each slice of the dragonfly body. The non-dimensional length and volume of each slice of the dragonfly model are recorded. The non-dimensional MOI included in the table are nondimensionalized by the body mass and length squared.

\begin{tabular}{|c|c|c|c|c|c|c|c|}
\hline & $\begin{array}{c}\text { Section } \\
\#\end{array}$ & $\ell_{i}$ & $V_{i}$ & $\begin{array}{l}I_{x x}{ }^{*} \\
\times 10^{4} \\
\end{array}$ & $\begin{array}{l}I_{y y}{ }^{*} \\
\times 10^{4} \\
\end{array}$ & $I_{z z}^{*} \times 10^{4}$ & $\begin{array}{l}I_{x z}{ }^{*} \\
\times 10^{4} \\
\end{array}$ \\
\hline \multirow{3}{*}{ Head } & 1 & $3.93 \mathrm{E}-02$ & $2.00 \mathrm{E}-04$ & \multirow{7}{*}{15.00} & \multirow{7}{*}{97.00} & \multirow{7}{*}{96.00} & \multirow{7}{*}{-0.44} \\
\hline & 2 & $5.71 \mathrm{E}-02$ & $6.24 \mathrm{E}-04$ & & & & \\
\hline & 3 & $6.81 \mathrm{E}-03$ & 3.69E-05 & & & & \\
\hline \multirow{4}{*}{ Thorax } & 4 & $3.52 \mathrm{E}-02$ & $2.76 \mathrm{E}-04$ & & & & \\
\hline & 5 & $5.76 \mathrm{E}-02$ & 7.79E-04 & & & & \\
\hline & 6 & $6.63 \mathrm{E}-02$ & $9.03 \mathrm{E}-04$ & & & & \\
\hline & 7 & $8.66 \mathrm{E}-02$ & $6.85 \mathrm{E}-04$ & & & & \\
\hline \multirow{3}{*}{ Inner Tail } & 8 & $4.27 \mathrm{E}-02$ & $2.46 \mathrm{E}-04$ & \multirow{14}{*}{1.50} & \multirow{14}{*}{230.80} & \multirow{14}{*}{230.50} & \multirow{14}{*}{-6.80} \\
\hline & 9 & $6.59 \mathrm{E}-02$ & 4.10E-04 & & & & \\
\hline & 10 & $3.91 \mathrm{E}-02$ & $1.40 \mathrm{E}-04$ & & & & \\
\hline \multirow{12}{*}{ Outer Tail } & 11 & $3.76 \mathrm{E}-02$ & $7.71 \mathrm{E}-05$ & & & & \\
\hline & 12 & 4.19E-02 & $6.70 \mathrm{E}-05$ & & & & \\
\hline & 13 & $5.02 \mathrm{E}-02$ & $6.00 \mathrm{E}-05$ & & & & \\
\hline & 14 & $5.86 \mathrm{E}-02$ & $8.12 \mathrm{E}-05$ & & & & \\
\hline & 15 & $4.93 \mathrm{E}-02$ & $6.46 \mathrm{E}-05$ & & & & \\
\hline & 16 & $5.11 \mathrm{E}-02$ & $9.45 \mathrm{E}-05$ & & & & \\
\hline & 17 & $5.40 \mathrm{E}-02$ & $1.01 \mathrm{E}-04$ & & & & \\
\hline & 18 & $3.43 \mathrm{E}-02$ & $7.16 \mathrm{E}-05$ & & & & \\
\hline & 19 & $5.89 \mathrm{E}-02$ & $1.16 \mathrm{E}-04$ & & & & \\
\hline & 20 & $3.42 \mathrm{E}-02$ & $5.05 \mathrm{E}-05$ & & & & \\
\hline & 21 & $3.32 \mathrm{E}-02$ & $1.91 \mathrm{E}-05$ & & & & \\
\hline & Total & $1.00 \mathrm{E}+00$ & $5.10 \mathrm{E}-03$ & 16.5 & \begin{tabular}{|l|l|}
327.8 \\
\end{tabular} & 326.5 & -7.24 \\
\hline
\end{tabular}

A

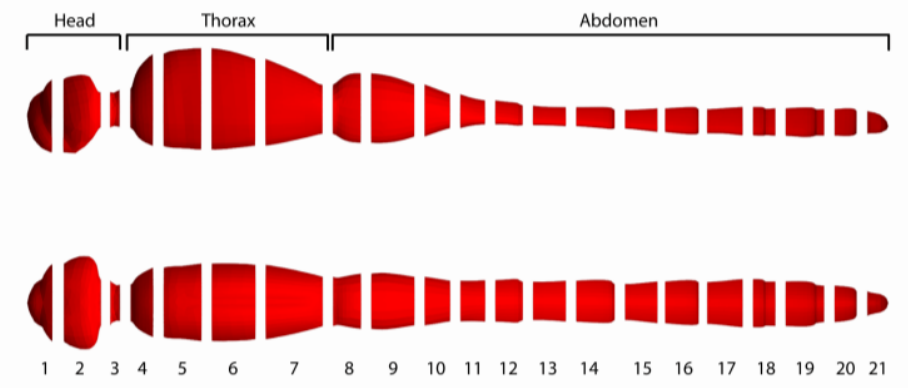

B
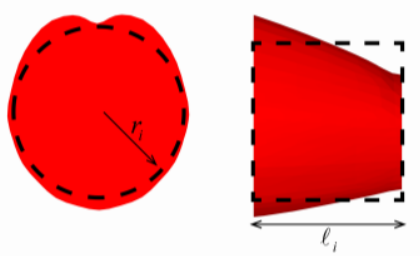

Figure 23: Dragonfly model for MOI calculations. Figure A depicts a sliced dragonfly model. The model is sliced into 21 pieces. Slices 1-3, 4-7, 8-21 make up the head, thorax, and abdomen, respectively. Figure B shows the approximation of an $\mathrm{i}^{\text {th }}$ slice as a cylinder having a volume equivalent to the slice. $\mathrm{r}_{\mathrm{i}}$ shows the corresponding radius of the approximated cylinder and $l_{i}$ shows the length of the cylinder. The length of the cylinder is equal to the length of each slice. 
From the 3D model, we obtained the non-dimensional geometric center of mass as well as the volume of each slice. Afterward, each individual slice was approximated as a cylinder with a volume equivalent to the actual slice volume to quantify the contribution of the slices to the MOI. The equivalent cylinder has the same length as the slice and the circular cross-sectional area is oriented in the direction of the roll axis. The mass of each slice is computed by sectioning a real dragonfly into three pieces; head, thorax and abdomen. We assumed that density is constant across each section. The real insect body length is used to dimensionalize all the length quantities. From this dimensionalization, we obtain the dimensional volume of each slice and then multiply density by volume to obtain the mass of each slice. The tensor of MOI of the dragonfly was calculated by adding the MOI of each slice about the axes fixed at the center of mass of the body. $I_{\text {head }}^{\prime}, I_{\text {thorax }}^{\prime}, I_{\text {tail }}^{\prime}$ are tensors of moment of inertia of the slices of the head, thorax, tail, respectively, about the axes originating from their own center of mass and fixed to them. $I_{\text {trans }_{i}}$ represents the tensor which contains the parallel axis theorem that enables us to calculate the effect of distance from the body center of mass to the center of mass of each individual slice.

$$
\begin{array}{cc}
I=\sum_{i \in n} I_{i}^{\prime}+I_{\text {trans }_{i}} & n=\{\text { head,thorax, abdomen }\} \\
I_{i}{ }^{\prime}=\frac{m_{i}}{12}\left[\begin{array}{ccc}
6 r_{i}^{2} & 0 & 0 \\
0 & 3 r_{i}^{2}+l_{i}^{2} & 0 \\
0 & 0 & 3 r_{i}^{2}+l_{i}^{2}
\end{array}\right] & I_{\text {trans }_{i}}=m_{i}\left[\begin{array}{ccc}
y_{i}^{2}+z_{i}^{2} & x_{i} y_{i} & x_{i} z_{i} \\
y_{i} x_{i} & x_{i}^{2}+z_{i}^{2} & y_{i} z_{i} \\
z_{i} x_{i} & z_{i} y_{i} & x_{i}^{2}+y_{i}^{2}
\end{array}\right]
\end{array}
$$

\subsubsection{Optimization Problem Definition}

Our objective in defining the optimization problem was to find out whether body bending patterns during maneuvering fight of dragonflies are beneficial to the dynamics of the system or are rather passive reactions. In other words, if the system is free to stay rigid or deflect during the maneuver will it choose to deflect and if so how this deflection will change during the course of the maneuver or 
how would it benefit the insect? To investigate, we defined an optimization problem with the objective of minimizing the average total flight torque needed to perform a specific maneuver. We modeled the tail as a free bidimensional pendulum so that the dragonfly can continuously deflect its tail in any direction at any time and change it during the course of the maneuver, if desired. We used a 4-control point B-spline curve to represent time history of angle of rotation. Similarly, each of three components of the axis of rotation of the tail is expressed by its respective B-spline curve. Each B-spline has two fixed and two variable control points and optimization algorithm is able to locate four pair of control points (corresponding to four B-splines) which finally define time history of change in axis and angle of rotation of the tail. Both the schematic for the B-spline curves as well as the optimization flow chat is show in Figure 24. For a known maneuver, the instantaneous magnitude of the total flight torque can be calculated as follows:

$$
\vec{\tau}_{\text {total }}=I \overrightarrow{\dot{\omega}}+\vec{\omega} \times I \vec{\omega}+\dot{I} \vec{\omega}
$$

Where $\tau_{\text {total }}$ is the vector of flight torque. The first two terms on the right hand side of Eqn. (4)comprise the aerodynamic torque and the last term is an inertial torque due to the tail deflection. Note that that so called aerodynamic torque in this study, includes some inertial terms due to coupling between the three rotational motions but an investigation into the coupling is beyond the scope of this work. A detailed discussion on those inertial terms due to coupling and their effect on the dynamics of flight can be found in Ref. [99].

The cost function is directly correlated with energy expenditure in flight may be defined to minimize the average or the maximum flight torque. The cost function chosen in this study is calculated as,

$$
\cos t=\left|\operatorname{mean}\left(\tau_{\text {pitch }}\right)+\operatorname{mean}\left(\tau_{\text {yaw }}\right)\right|
$$

Where $\tau_{\text {pitch }}$ and $\tau_{\text {yaw }}$ are the aerodynamic components of the flight torque in yaw and pitch directions. The optimization was performed using a built-in optimizer (fmincon) in MATLAB (Mathworks, Natick, MA, USA). We chose "interior point" as the search algorithm. 
A

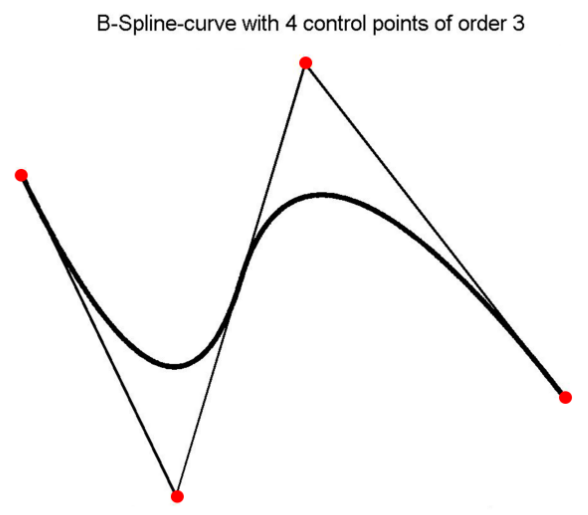

B

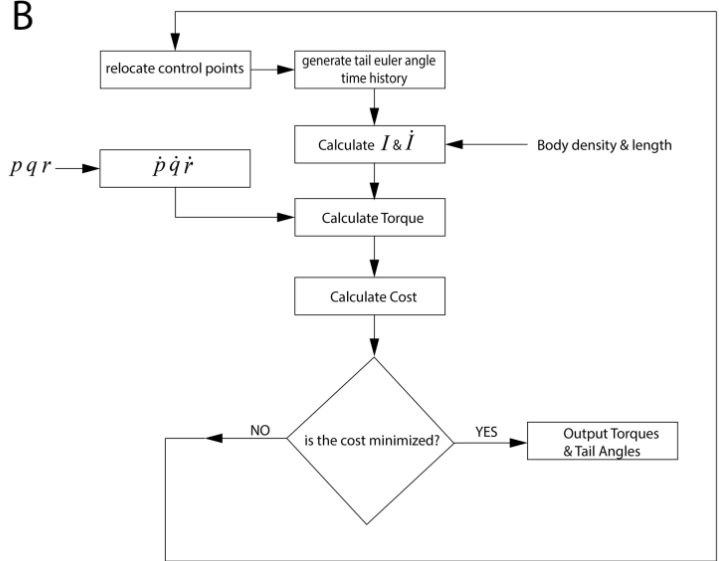

Figure 24: Optimization Problem Definition. Figure 24A shows a third order B-spline curve with 4 control points. The control points are depicted as red circles. The B-spline curve help generate the time history of the tail euler angles. Figure 24B represents the optimization flow chart. The inputs into the optimization algorithm are body density and length as well as body angular velocities, pqr. The volume of each slice of the dragonfly is obtained by dimensionalizing the non-dimensional volume by body length. Having the mass distribution, MOI and time rate of change of MOI can be calculated. The time rate of change of body angular velocities can be calculated also. The torque is calculated given angular velocities, rate of change of MOI, and rate of change of MOI. The cost function then calculates the energy expenditure. If the energy expenditure is minimal, the optimization algorithm output the tail angles and torques. Otherwise, the optimization is iterated by relocating the control points on the B-spline curves and the generating new tail euler angles. The process iterates till minimal energy expenditure is attained.

\subsection{Results and Discussion}

\subsubsection{Effect of Tail Deflection on Changes in MOI}

As aforementioned in the introduction, by deflecting the tail, the dragonfly alters the moment of inertia tensor. In order to quantify effect of the tail deflection on the MOI tensor changes, we varied the tail pitch angle between $-45^{\circ}$ to $45^{\circ}$ while keeping the tail yaw angle constant and vice versa. The ranges of tail deflections are chosen based on free flight observations of dragonflies. In both cases, we observed that by deflecting the tail, pitchwise or yawwise, the moment of inertia around the yaw and pitch axis can be enhanced up to twice its original value. At erect posture, the roll moment of inertia of a dragonfly body is about 2 orders of magnitude smaller than the yaw or pitch MOI. This suggests that the response of the dragonfly body to the roll torque is significantly faster than that of the pitch or yaw. This may cause discrepancies in the sensitivity of the insect motion sensors. By deflecting the tail, $\mathrm{I}_{\mathrm{xx}}$ 
can be increased more than 20 times its original value which may be necessary for controlling the roll motion (Figure 25A and B). We also observed that the products of inertia were also influenced by yawing and pitching the tail. The products of inertia are measures of body symmetry. When the tail is deflected pitchwise, there is an increase in asymmetry in the xz plane. Likewise when the yawwise deflection occurs, there is asymmetry in the xy plane. The results of our analysis in this section indicate that the variations in the MOI tensor can be potentially significant during the flight.
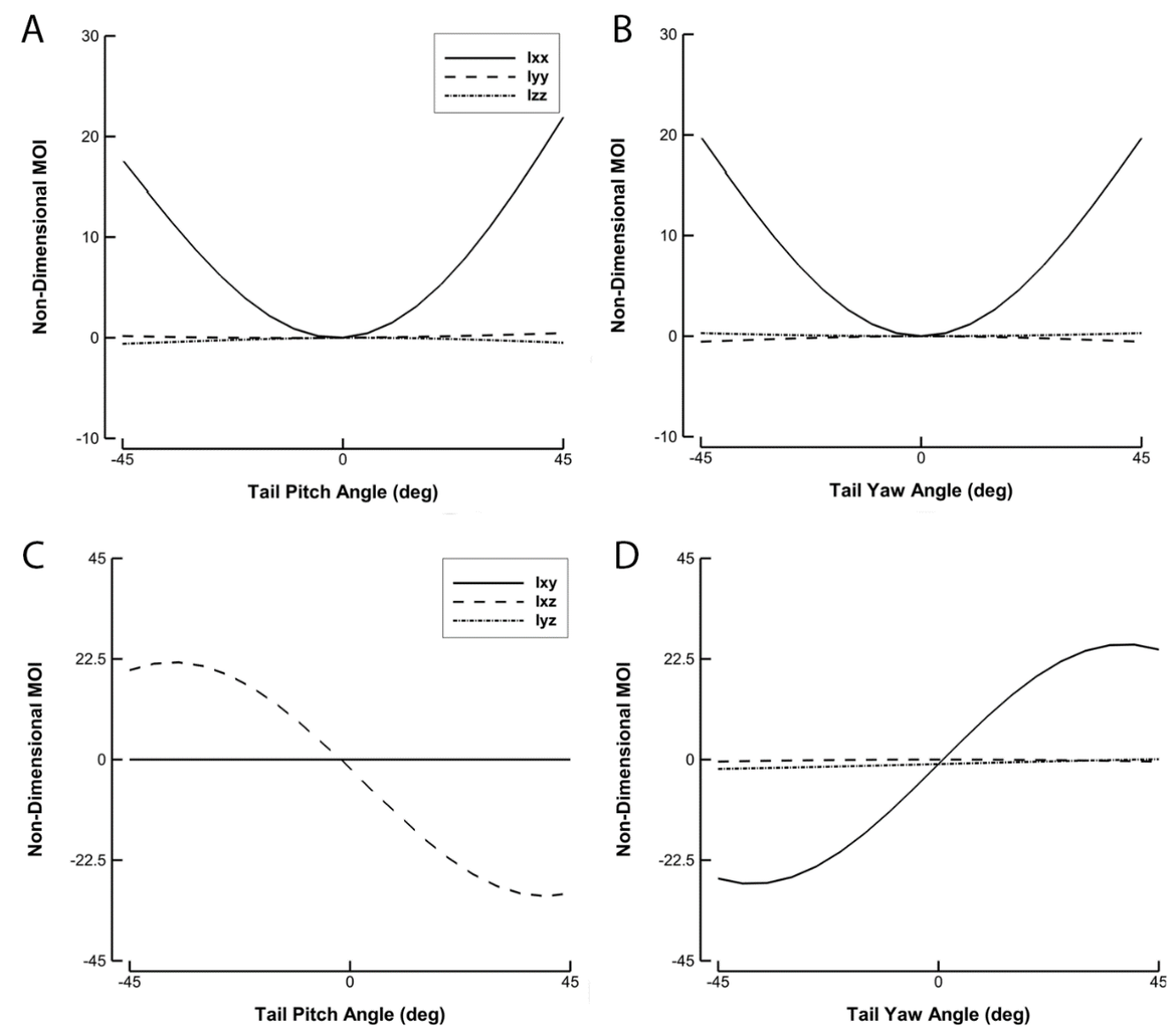

Figure 25: Effect of variation of tail Euler angles on MOI. The figures (A, B, C and D) show the amount of change that takes place in the moment of inertia when the tail is pitched only or yawed only. The tail angles are varied between -45 to 45 degrees. 0 degrees represents the erect position of the dragonfly. The top two figures, A and B, illustrate that when the moment of inertia is varied in pitch and yaw axis, the greatest variation occurs in the rolling moment of inertia. The rolling moment of inertia was about 20 times its original value while the pitching and yawing MOI did not change much. The bottom figures, $\mathrm{C}$ and $\mathrm{D}$, also illustrate the change in body symmetry when the tail is bent pitch wise or yaw wise. 


\subsubsection{Optimized Tail Deflection and its Relationship to Body Yaw Velocity.}

To investigate the effect of tail bending on the overall torque generation in dragonfly maneuvering flight, we first used the real Euler angles of a dragonfly in a turning takeoff maneuver and calculated the optimized posture of the tail during that flight. A turning takeoff is a flight maneuver during which a dragonfly changes the flight heading by more than 90 degrees while the center of mass is elevated over several body lengths $[99,103]$. The body motion reconstruction is performed using an accurate method which is described comprehensively in Ref. [63]. Figure 26A, shows the body angular velocities, the optimized tail angles and the total torque (thin solid line), aerodynamic torque (dashed line) and rigid body torque (thick solid line). The rigid body torque is calculated by balancing the dynamics of the motion using the known values of body angular velocities and accelerations as well the moment of inertia of the rigid and erect dragonfly body. The acceleration and deceleration phase of the maneuver is respectively defined as the duration before and after the maximum body yaw velocity was attained. The acceleration phase is shaded in Figure 26 for clarity. During the maneuver, both the yawwise and pitchwise deflections of the tail increases as the body yaw velocity increased. The maximum deflection in the tail yaw angle was reached at the point that the body yaw velocity was maximum. The maximum pitchwise deflection of the tail happened earlier during the maneuver, similar to the occurrence of the maximum pitch velocity of the body. One important observation is that the total torque required by a rigid body is substantially different from that of a flexible body with an optimized posture. Note that if the body is rigid, there is only one single solution to Eqn. (4), meaning that if $\vec{\omega}$ and consequently $\overrightarrow{\dot{\omega}}$ are known, there is only one solution for torque. However, for a flexible body there are infinite ways of executing the same motion. Body flexibility can change the flight torque by two means. First, by offering inertial torque terms due to the tail deflection; $\dot{I} \vec{\omega}$, and second, by manipulating the instantaneous magnitude of each element in the tensor of MOI. Comparing the yaw torque for a dragonfly with a rigid body (thick solid blue line) with that of one with a flexible body (thin solid blue line), we can infer that that the yaw toque of the flexible body is significantly lower 
than that of a rigid body, especially during the acceleration phase. Similarly, the pitch torque of a rigid body stays high throughout the maneuver while that of the flexible body drops to substantially smaller magnitudes during early stages of the flight. The roll torque of the flexible body with the optimized body posture is larger than that of the rigid body. That is due to our choice of cost function in this study which assumes the cost of rolling the body is significantly smaller than that of yawing or pitching. The cost function can be modified to incorporate a cost to generate roll torque as well. The difference between the thin solid line and dashed line in Figure 26 shows the contribution of the inertial torque term due to the tail deflection; the last term on the right hand side of Eqn. (4). It is evident from Figure 26 that inertial term is close in magnitude to the aerodynamic torque. Note that as the tail bends, it does not always generate inertial torque that is in favor of the maneuver. In fact, during the acceleration phase, the inertial torque due to the tail deflection decreased the total torque. In interpreting these results one needs to remember, as was previously mentioned, that this inertial tem is not the only contribution of the body flexibility to the flight torque and the overall benefit of the tail bending can only be understood by considering all three kinds of lines; thick solid, thin solid and dashed, that are plotted in torque graph. Furthermore, the maximum deflection of the tail is only about 15 degrees yawwise and 10 degrees pitchwise. These results imply that small abdominal deflections during maneuvering flight are enough to enhance the flight performance. 
A

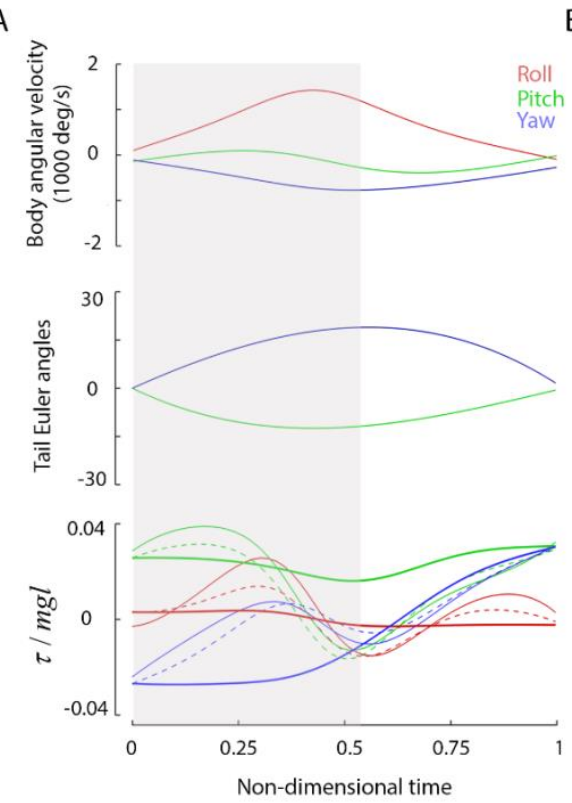

B

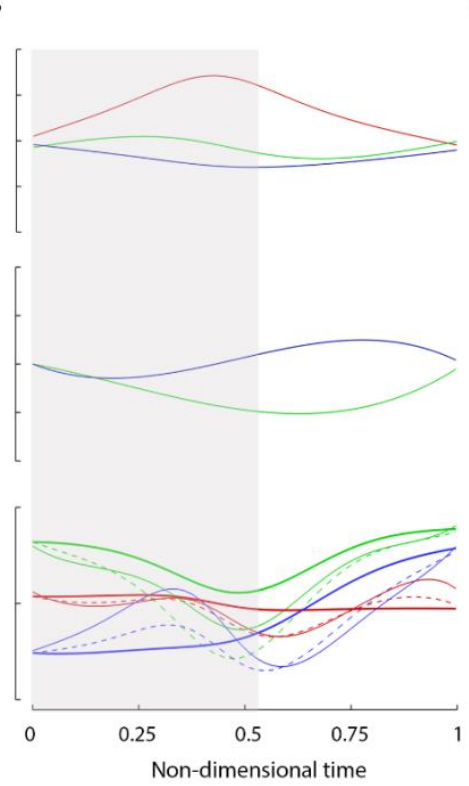

C

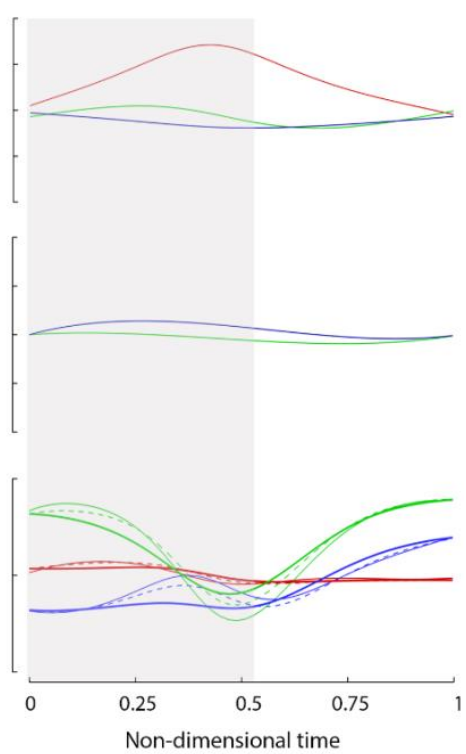

Figure 26: Optimized tail bending kinematics and the resultant flight torque. This figure (A, B and C) comprises of body angular velocity data, the optimized tail angles as well as the torques obtained from the cost function. Figure A, represent optimized results based on real insect flight data while B and C represent data obtained when the body yaw velocity was decreased to $75 \%$ and then $50 \%$, respectively. In the torque plots shown on the third row, the thick solid lines represent the rigid body torques, the thin dashed lines represent the aerodynamic torque and the thin solid lines represent the total flight torque.

To investigate the connection between the optimized body posture and the flight, we systematically altered the body yaw velocity and solved for the optimized tail deflection. The body yaw velocity was decreased to $75 \%$ and then $50 \%$ and the results are shown in Figure $26 \mathrm{~B}$ and C. Visual inspection of Figure 26 immediately indicates that the magnitude of the tail deflection is directly correlated with the magnitude of the body yaw velocity; meaning that more deflections are required for faster maneuvers. The other important observation is that both tail yaw and pitch angles vary accordingly with the yaw velocity even though the pitch velocity is identical for all three cases represented in Figure 26. In all three cases in Figure 26, the magnitude of the inertial torque due to the tail bending is smaller in acceleration phase when compared to deceleration phase.

\subsection{Conclusions}

Taken together, our results imply that body flexibility benefits the flight performance by offering more ways by which a flight can be achieved. This is accomplished by controlling the 
instantaneous mass distribution of the body as well as the generating inertial torques due to movement of the tail with respect to the thorax. The former changes the response of the body to the generated torque and enhances or decreases the resistance of the body to a specific motion. The latter acts by inserting inertial terms that are proportional to the rate of change in the mass distribution. Our results indicates that both these effects play an essential role in reducing the average flight torque required for the flight with the former effect being more dominant. Having tackled the tail deflection problem comprehensively, there are still many questions to be investigated. In the future, we hope to investigate the influence of body shape on the optimized body posture. For example, insects with round and stocky bodies are less commonly observed to deflect their abdomen during flight. Although, this may be related to the body morphological limitations, we expect that the body geometry as well as the mass distribution would influence the optimized results.

\subsection{Acknowledgments}

This work was partially supported by NSF [grant number CEBT-1313217].

\subsection{Accomplishments}

\section{Chapter 5: Summary and Conclusions}

Investigations were carried out on three different flight modes of four-winged fliers; flight initiation (takeoff), backward flight and maneuvers in order to understand the axioms which best characterize these flight modes. In the literature, most well-studied flight modes are forward flight, hovering and maneuvers. Hence, the flight mechanisms of insects cannot be completely understood until other commonly used mechanism by insects are elucidated. Here, for the first time, we studied new flight modes; takeoff and backward flight. As insects flap their wings, they also deform their bodies. Hence, the role of body deformation in dragonfly flight was also clarified. The problems were approached from unique perspectives using computational fluid dynamic simulations and an optimization algorithm to provide new insights into the physics of flapping flight. The main questions answered in this thesis are as follows. 1) What happens to the aerodynamic mechanisms and flow 
features when the flight mode changes? Is the force generation mechanism universal? 2) Is the upstroke capable of producing substantial aerodynamics forces? Could those forces equal or exceed the downstroke forces? 3) If insects deform their bodies during flight, are there energy savings benefits? Is there an optimal configuration?

Based on the results on the works on flight initiation (takeoff) and backward flight, it was shown that aerodynamic mechanisms change based on the type of flight. During takeoff, the downstroke phase generated substantial forces for lift off. An LEV present on the wing surface helped boost force production. Likewise in the upstroke, large forces were produced. These forces were equal to the downstroke forces. An LEV, stably attached to the wing surface was responsible for large force generation. As the flight transitioned to a well-studied flight mode i.e. forward flight, the upstroke, did not contribute to force production substantially.

During backward flight, an upright body posture was a necessary condition for flight. Tilting of the stroke plane to the longitudinal axis of the body as well as reorienting the global positioning of the stroke plane were also important. The findings indicate that because of the upright body posture, the aerodynamic roles of the up and downstroke were reversed. In well-studied flight modes such as forward flight and hovering, the downstroke generated forces for weight support and the upstroke usually generated $10-20 \%$ of force and was restricted to generating thrust forces. During backward flight, the upstroke generates forces for weight support while the downstroke generates thrust forces. The upstroke can generate on average 2-3 times the body weight and the downstroke 1.5 times the body weight. An LEV was responsible for force generation in both halfstrokes but a stronger LEV was present in the upstroke. Hence, just as in flight initiation, clear evidence for an active upstroke was shown.

To close the loop, emphasis was also placed on body deformation which is observed as insects fly. It was shown than body deformations could be beneficial to flight by minimizing the aerodynamic torque (energy expenditure) needed to perform a maneuver. Hence the body deformations are not 
strictly restricted to serving as an aerodynamic rudder or fine tuning moments produced by the wings as was previously thought.

\subsection{Ongoing and Future Work}

Current ongoing work is to examine other flight modes wherein an active upstroke can be utilized such as ascending flight. Furthermore, in this work only straight backward flight was studied. However, insects can also perform maneuvers while reversing. It will be interesting to document the flight mechanism governing reverse maneuvers. The transition of forward to backward to forward flight is also of interest to me.

The current study on body deformation was limited because the torque calculation was obtained by solving Euler's rigid body dynamics equation. In the future, a high fidelity CFD simulation will used to compute the torque. The optimization algorithm can also be integrated into the in-house CFD solver used in this work.

Overall, I believe that elucidating the flight mechanics of insects in different flight modes showcased in this work is a giant leap toward designing tomorrows MAVs.

\section{Bibliography}

[1] Dickinson, M. 2006 Insect flight. Current Biology 16, R309-R314. (doi:http://dx.doi.org/10.1016/j.cub.2006.03.087).

[2] Anderson, J.D. 2012 Introduction to flight. New York, McGraw Hill.

[3] Igan, M. 2014 Earths Forbidden Secrets, CreateSpace Independent Publishing Platform.

[4] Dickinson, M.H., Lehmann, F.-O. \& Sane, S.P. 1999 Wing rotation and the aerodynamic basis of insect flight. Science 284, 1954-1960.

[5] Maxworthy, T. 1981 The fluid dynamics of insect flight. Annual Review of Fluid Mechanics 13, 329-350.

[6] Johansson, L.C., Engel, S., Kelber, A., Heerenbrink, M.K. \& Hedenström, A. 2013 Multiple leading edge vortices of unexpected strength in freely flying hawkmoth. Scientific reports 3.

[7] Sato, M. \& Azuma, A. 1997 The flight performance of a damselfly Ceriagrion melanurum Selys. Journal of Experimental Biology 200, 1765-1779.

[8] Ellington, C. 1984 The aerodynamics of hovering insect flight. I. The quasi-steady analysis. Philosophical Transactions of the Royal Society of London B: Biological Sciences 305, 1-15. [9] Liu, H., Ellington, C.P., Kawachi, K., Van Den Berg, C. \& Willmott, A.P. 1998 A computational fluid dynamic study of hawkmoth hovering. Journal of Experimental Biology 201, 461-477.

[10] Liu, G., Dong, H. \& Li, C. 2016 Vortex dynamics and new lift enhancement mechanism of wing-body interaction in insect forward flight. Journal of Fluid Mechanics 795, 634-651. 
[11] Viieru, D., Tang, J., Lian, Y., Liu, H. \& Shyy, W. 2006 Flapping and flexible wing aerodynamics of low Reynolds number flight vehicles. AIAA paper 503, 2006.

[12] Bomphrey, R.J., Nakata, T., Phillips, N. \& Walker, S.M. 2017 Smart wing rotation and trailingedge vortices enable high frequency mosquito flight. Nature advance online publication.

(doi:10.1038/nature21727

http://www.nature.com/nature/journal/vaop/ncurrent/abs/nature21727.html\#supplementaryinformation).

[13] Carmichael, B. 1981 Low Reynolds number airfoil survey, volume 1.

[14] Ellington, C.P. 2006 Insects versus birds: the great divide. In 44th AIAA aerospace sciences meeting and exhibit (pp. 1-6.

[15] Chin, D.D. \& Lentink, D. 2016 Flapping wing aerodynamics: from insects to vertebrates. Journal of Experimental Biology 219, 920-932.

[16] Muijres, F., Johansson, L., Barfield, R., Wolf, M., Spedding, G. \& Hedenström, A. 2008

Leading-edge vortex improves lift in slow-flying bats. Science 319, 1250-1253.

[17] Lentink, D. \& Dickinson, M.H. 2009 Rotational accelerations stabilize leading edge vortices on revolving fly wings. Journal of Experimental Biology 212, 2705-2719.

[18] Birch, J.M., Dickson, W.B. \& Dickinson, M.H. 2004 Force production and flow structure of the leading edge vortex on flapping wings at high and low Reynolds numbers. Journal of Experimental Biology 207, 1063-1072.

[19] Ellington, C.P., Van Den Berg, C., Willmott, A.P. \& Thomas, A.L. 1996 Leading-edge vortices in insect flight.

[20] Willmott, A.P., Ellington, C.P. \& Thomas, A.L. 1997 Flow visualization and unsteady aerodynamics in the flight of the hawkmoth, Manduca sexta. Philosophical Transactions of the Royal Society B: Biological Sciences 352, 303-316.

[21] Thomas, A.L., Taylor, G.K., Srygley, R.B., Nudds, R.L. \& Bomphrey, R.J. 2004 Dragonfly flight: free-flight and tethered flow visualizations reveal a diverse array of unsteady lift-generating mechanisms, controlled primarily via angle of attack. Journal of Experimental Biology 207, 42994323.

[22] Lu, Y., Shen, G.X. \& Lai, G.J. 2006 Dual leading-edge vortices on flapping wings. Journal of experimental biology 209, 5005-5016.

[23] Lu, Y. \& Shen, G.X. 2008 Three-dimensional flow structures and evolution of the leading-edge vortices on a flapping wing. Journal of Experimental Biology 211, 1221-1230.

[24] Sane, S.P. 2003 The aerodynamics of insect flight. The journal of experimental Biology 206, 4191-4208.

[25] Ellington, C.P. 1999 The novel aerodynamics of insect flight: applications to micro-air vehicles. Journal of Experimental Biology 202, 3439-3448.

[26] Fry, S.N., Sayaman, R. \& Dickinson, M.H. 2005 The aerodynamics of hovering flight in Drosophila. Journal of Experimental Biology 208, 2303-2318.

[27] Wang, J.K. \& Sun, M. 2005 A computational study of the aerodynamics and forewing-hindwing interaction of a model dragonfly in forward flight. Journal of Experimental Biology 208, 3785-3804. (doi:10.1242/jeb.01852).

[28] Wang, Z.J. \& Russell, D. 2007 Effect of forewing and hindwing interactions on aerodynamic forces and power in hovering dragonfly flight. Physical review letters 99, 148101.

[29] CAMHI, J.M. 1970 Yaw-correcting postural changes in locusts. Journal of Experimental Biology 52, 519-531.

[30] Camhi, J.M. 1970 Sensory control of abdomen posture in flying locusts. J. exp. Biol 52, 533.

[31] Zanker, J. 1988 On the mechanism of speed and altitude control in Drosophila melanogaster.

Physiological entomology 13, 351-361.

[32] Dyhr, J.P., Morgansen, K.A., Daniel, T.L. \& Cowan, N.J. 2013 Flexible strategies for flight control: an active role for the abdomen. Journal of Experimental Biology 216, 1523-1536. 
[33] Luu, T., Cheung, A., Ball, D. \& Srinivasan, M.V. 2011 Honeybee flight: a novel 'streamlining'response. Journal of Experimental Biology 214, 2215-2225.

[34] Berthé, R. \& Lehmann, F.-O. 2015 Body appendages fine-tune posture and moments in freely manoeuvring fruit flies. The Journal of Experimental Biology 218, 3295-3307.

(doi:10.1242/jeb.122408).

[35] Taylor, G.K. 2001 Mechanics and aerodynamics of insect flight control. Biological Reviews 76, 449-471.

[36] Zeyghami, S., Bode-Oke, A.T. \& Dong, H. 2016 Effect of Body Configuration and Deformation on the dynamic stability of flapping flight. In AIAA Atmospheric Flight Mechanics Conference (p. 3540 .

[37] Phan, H.V., Nguyen, Q.V., Truong, Q.T., Van Truong, T., Park, H.C., Goo, N.S., Byun, D. \& Kim, M.J. 2012 Stable vertical takeoff of an insect-mimicking flapping-wing system without guide implementing inherent pitching stability. Journal of Bionic Engineering 9, 391-401.

[38] Pond, C.M. 1972 The initiation of flight in unrestrained locusts,Schistocerca gregaria. Journal of comparative physiology 80, 163-178. (doi:10.1007/bf00696488).

[39] Burrows, M. \& Morris, O. 2003 Jumping and kicking in bush crickets. Journal of Experimental Biology 206, 1035-1049.

[40] Card, G. \& Dickinson, M. 2008 Performance trade-offs in the flight initiation of Drosophila. Journal of Experimental Biology 211, 341-353. (doi:10.1242/jeb.012682).

[41] Burrows, M. 2007 Kinematics of jumping in leafhopper insects (Hemiptera, Auchenorrhyncha, Cicadellidae). Journal of Experimental Biology 210, 3579-3589. (doi:10.1242/jeb.009092).

[42] Crandell, K.E. \& Tobalske, B.W. 2015 Kinematics and aerodynamics of avian upstrokes during slow flight. The Journal of experimental biology 218, 2518-2527.

[43] Tobalske, B.W., Altshuler, D.L. \& Powers, D.R. 2004 Take-off mechanics in hummingbirds (Trochilidae). Journal of Experimental Biology 207, 1345-1352.

[44] Chen, M.W., Zhang, Y.L. \& Sun, M. 2013 Wing and body motion and aerodynamic and leg forces during take-off in droneflies. Journal of The Royal Society Interface 10, 20130808.

[45] Truong, T., Le, T., Park, H., Yoon, K., Kim, M. \& Byun, D. 2014 Non-jumping take off performance in beetle flight (Rhinoceros Beetle Trypoxylus dichotomus), J. Bionic Eng 11, 61-71. [46] Marden, J.H. 1987 Maximum lift production during takeoff in flying animals. J. exp. Biol 130, 235-238.

[47] Rüppell, G. 1989 Kinematic analysis of symmetrical flight manoeuvres of Odonata. Journal of Experimental Biology 144, 13-42.

[48] Alexander, D.E. 1984 Unusual phase relationships between the forewings and hindwings in flying dragonflies. Journal of Experimental Biology 109, 379-383.

[49] Wang, Z.J. 2005 Dissecting insect flight. Annu. Rev. Fluid Mech. 37, 183-210.

[50] Azuma, A., Azuma, S., Watanabe, I. \& Furuta, T. 1985 Flight mechanics of a dragonfly. Journal of experimental biology 116, 79-107.

[51] Norberg, R.A. 1975 Hovering flight of the dragonfly Aeschna juncea L., kinematics and aerodynamics. In Swimming and flying in nature (pp. 763-781, Springer.

[52] Wakeling, J. \& Ellington, C. 1997 Dragonfly flight. III. Lift and power requirements. Journal of Experimental Biology 200, 583-600.

[53] David, C.T. 1978 The relationship between body angle and flight speed in free-flying Drosophila. Physiological Entomology 3, 191-195. (doi:10.1111/j.1365-3032.1978.tb00148.x).

[54] Bomphrey, R.J., Taylor, G.K. \& Thomas, A.L.R. 2009 Smoke visualization of free-flying bumblebees indicates independent leading-edge vortices on each wing pair. Experiments in Fluids 46, 811-821. (doi:10.1007/s00348-009-0631-8).

[55] Bomphrey, R.J., Lawson, N.J., Harding, N.J., Taylor, G.K. \& Thomas, A.L.R. 2005 The aerodynamics of Manduca sexta: digital particle image velocimetry analysis of the leading-edge vortex. Journal of Experimental Biology 208, 1079-1094. (doi:10.1242/jeb.01471). 
[56] Bomphrey, R.J., Nakata, T., Henningsson, P. \& Lin, H.-T. 2016 Flight of the dragonflies and damselflies. Philosophical Transactions of the Royal Society B: Biological Sciences 371.

(doi:10.1098/rstb.2015.0389).

[57] Sunada, S., Kawachi, K., Watanabe, I. \& Azuma, A. 1993 Performance of a butterfly in take-off flight. Journal of Experimental Biology 183, 249-277.

[58] Ellington, C. 1984 The aerodynamics of hovering insect flight. IV. Aeorodynamic mechanisms. Philosophical Transactions of the Royal Society of London B: Biological Sciences 305, 79-113.

[59] Zeyghami, S. \& Dong, H. 2015 Coupling of the wings and the body dynamics enhances damselfly maneuverability. arXiv preprint arXiv:1502.06835.

[60] Spedding, G.R. \& Hedenström, A. 2009 PIV-based investigations of animal flight. Experiments in Fluids 46, 749-763.

[61] Hubel, T.Y., Hristov, N.I., Swartz, S.M. \& Breuer, K.S. 2009 Time-resolved wake structure and kinematics of bat flight. Experiments in fluids 46, 933-943.

[62] Young, J., Walker, S.M., Bomphrey, R.J., Taylor, G.K. \& Thomas, A.L.R. 2009 Details of Insect Wing Design and Deformation Enhance Aerodynamic Function and Flight Efficiency. Science 325, 1549-1552. (doi:10.1126/science.1175928).

[63] Koehler, C., Liang, Z., Gaston, Z., Wan, H. \& Dong, H. 2012 3D reconstruction and analysis of wing deformation in free-flying dragonflies. The Journal of experimental biology 215, 3018-3027.

[64] Zeyghami, S., Babu, N. \& Dong, H. 2016 Cicada (Tibicen linnei) steers by force vectoring.

Theoretical and Applied Mechanics Letters 6, 107-111.

[65] Wan, H., Dong, H. \& Gai, K. 2015 Computational investigation of cicada aerodynamics in forward flight. Journal of The Royal Society Interface 12, 20141116.

[66] Hunt, J.C., Wray, A. \& Moin, P. 1988 Eddies, streams, and convergence zones in turbulent flows.

[67] Wakeling, J. \& Ellington, C. 1997 Dragonfly flight. II. Velocities, accelerations and kinematics of flapping flight. Journal of experimental biology 200, 557-582.

[68] Lehmann, F.-O. 2009 Wing-wake interaction reduces power consumption in insect tandem wings. Experiments in fluids 46, 765-775.

[69] Sun, M. \& Huang, H. 2007 Dragonfly forewing-hindwing interaction at various flight speeds and wing phasing. AIAA journal 45, 508-511.

[70] Dudley, R. \& Ellington, C.P. 1990 Mechanics of Forward Flight in Bumblebees: II. Quasi-

Steady Lift and Power Requirements. Journal of Experimental Biology 148, 53-88.

[71] Sapir, N. \& Dudley, R. 2012 Backward flight in hummingbirds employs unique kinematic adjustments and entails low metabolic cost. The Journal of experimental biology 215, 3603-3611.

[72] Davey, W.N. \& Davey, J. 1945 Hovering and backward flight in the hummingbird. Jack Pine Warbler 23, 2-7.

[73] Williams, A.B. 1940 Acadian Flycatcher Flies Backward. The Auk 57, 255-255.

[74] Ng'weno, F. 1990 Sunbird flying backwards? East Africa Nat. Hist. Bull. 20, 27-28.

[75] D'Août, K. \& Aerts, P. 1999 A kinematic comparison of forward and backward swimming in the eel Anguilla anguilla. The Journal of experimental biology 202, 1511-1521.

[76] Lannoo, M.J. \& Lannoo, S.J. 1993 Why do electric fishes swim backwards? An hypothesis based on gymnotiform foraging behavior interpreted through sensory constraints. Environmental biology of fishes 36, 157-165.

[77] Olberg, R., Seaman, R., Coats, M. \& Henry, A. 2007 Eye movements and target fixation during dragonfly prey-interception flights. Journal of Comparative Physiology A 193, 685-693.

[78] Yokoi, T. \& Fujisaki, K. 2009 Hesitation behaviour of hoverflies Sphaerophoria spp. to avoid ambush by crab spiders. Naturwissenschaften 96, 195-200.

[79] Zeil, J. \& Wittmann, D. 1989 Visually controlled station-keeping by hovering guard bees ofTrigona (Tetragonisca) angustula (Apidae, Meliponinae). Journal of Comparative Physiology A 165, 711-718. 
[80] Mukundarajan, H., Bardon, T.C., Kim, D.H. \& Prakash, M. 2016 Surface tension dominates insect flight on fluid interfaces. Journal of Experimental Biology 219, 752-766.

(doi:10.1242/jeb.127829).

[81] Bode-Oke, A.T., Zeyghami, S. \& Dong, H. 2016 Strategies for Achieving Backward Flight by a Dragonfly. In 30th Congress of the International Council of the Aeronautical Sciences (Daejeon, South Korea.

[82] Sane, S.P., Dieudonné, A., Willis, M.A. \& Daniel, T.L. 2007 Antennal mechanosensors mediate flight control in moths. science 315, 863-866.

[83] Dong, H., Koehler, C., Liang, Z., Wan, H. \& Gaston, Z. 2010 An integrated analysis of a dragonfly in free flight. In 40th AIAA fluid dynamics conference and exhibit, AIAA2010-4390 ( [84] Ren, Y., Dong, H., Deng, X. \& Tobalske, B. 2016 Turning on a dime: Asymmetric vortex formation in hummingbird maneuvering flight. Physical Review Fluids 1, 050511.

[85] Bode-Oke, A.T., Zeyghami, S. \& Dong, H. 2015 Optimized Body Deformation in Dragonfly Maneuvers. In American Institute of Aeronautics and Astronautics Student Region I Conference ( [86] Wang, J., D. Russell. 2007 Effect of forewing and hindwing interactions on aerodynamic forces and power in hovering dragonfly flight. Phys. Rev. Lett. 99, 148101.

[87] Sun, M. \& Lan, S.L. 2004 A computational study of the aerodynamic forces and power requirements of dragonfly (Aeschna juncea) hovering. J. Exp. Biol. 207, 1887-1901.

[88] Wakeling, J.M. \& Ellington, C.P. 1997 Dragonfly flight. II. Velocities, accelerations and kinematics of flapping flight. J. Exp. Biol. 200, 557-582.

[89] Warrick, D.R., Tobalske, B.W. \& Powers, D.R. 2009 Lift production in the hovering hummingbird. Proceedings of the Royal Society of London B: Biological Sciences 276, 3747-3752. [90] Song, J., Luo, H. \& Hedrick, T.L. 2014 Three-dimensional flow and lift characteristics of a hovering ruby-throated hummingbird. Journal of The Royal Society Interface 11, 20140541. [91] Dudley, R. 2002 The biomechanics of insect flight: form, function, evolution. Princeton, Princeton University Press.

[92] A. J. Bergou, L.R., J. Guckenheimer, I. Cohen, Z.J. Wang. 2010 Fruit Fly Modulate Passive Wing Pitching to Generate In-Flight Turn. Phys. Rev. Lett. 104.

[93] Cheng, B., Fry, S., Huang, Q., Deng, X. 2009 Dynamics and Control of Turning in Fruit Fly Drosophila. Journal of Experimental Biology, In press.

[94] Götz, K.G., Hengstenberg, B. \& Biesinger, R. 1979 Optomotor control of wing beat and body posture in Drosophila. Biological Cybernetics 35, 101-112.

[95] Hinterwirth, A.J. \& Daniel, T.L. 2010 Antennae in the hawkmoth Manduca sexta (Lepidoptera, Sphingidae) mediate abdominal flexion in response to mechanical stimuli. Journal of Comparative Physiology A 196, 947-956.

[96] Dyhr, J.P., Morgansen, K.A., Daniel, T.L. \& Cowan, N.J. 2013 Flexible strategies for flight control: an active role for the abdomen. The Journal of experimental biology 216, 1523-1536.

[97] Finio, B.M. \& Wood, R.J. 2010 Distributed power and control actuation in the thoracic mechanics of a robotic insect. Bioinspiration \& biomimetics 5, 045006.

[98] Zeyghami, S. \& Dong, H. 2012 A Body Reorientation Strategy in Insect Takeoff Flight. In 50th AIAA Aerospace Sciences Meeting (

[99] Zeyghami, S. \& Dong, H. 2015 Study of turning takeoff maneuver in free-flying dragonflies:

effect of dynamic coupling. arXiv preprint arXiv:1502.06858.

[100] Combes, S.A. \& Dudley, R. 2009 Turbulence-driven instabilities limit insect flight performance. Proceedings of the National Academy of Sciences 106, 9105-9108.

(doi:10.1073/pnas.0902186106).

[101] Hedrick, T.L. \& Daniel, T.L. 2006 Flight control in the hawkmoth Manduca sexta: the inverse problem of hovering. Journal of Experimental Biology 209, 3114-3130.

[102] Taylor, G.K. \& Thomas, A.L.R. 2003 Dynamic flight stability in the desert locust Schistocerca gregaria. Journal of Experimental Biology 206, 2803-2829.

[103] Zeyghami, S. \& Dong, H. 2012 A Body Reorientation Strategy in Insect Takeoff Flight. 
